\title{
Quasichemical Models of Multicomponent Nonlinear Diffusion
}

\author{
A. N. Gorban ${ }^{1 *}$, H. P. Sargsyan ${ }^{2}$ and H. A. Wahab ${ }^{1}$ \\ ${ }^{1}$ Department of Mathematics, University of Leicester, Leicester, LE1 7RH, UK \\ ${ }^{2}$ UNESCO Chair - Life Sciences International Postgraduate \\ Educational Center (LSIPEC), Yerevan, Republic of Armenia
}

\begin{abstract}
Diffusion preserves the positivity of concentrations, therefore, multicomponent diffusion should be nonlinear if there exist non-diagonal terms. The vast variety of nonlinear multicomponent diffusion equations should be ordered and special tools are needed to provide the systematic construction of the nonlinear diffusion equations for multicomponent mixtures with significant interaction between components. We develop an approach to nonlinear multicomponent diffusion based on the idea of the reaction mechanism borrowed from chemical kinetics.

Chemical kinetics gave rise to very seminal tools for the modeling of processes. This is the stoichiometric algebra supplemented by the simple kinetic law. The results of this invention are now applied in many areas of science, from particle physics to sociology. In our work we extend the area of applications onto nonlinear multicomponent diffusion.

We demonstrate, how the mechanism based approach to multicomponent diffusion can be included into the general thermodynamic framework, and prove the corresponding dissipation inequalities. To satisfy thermodynamic restrictions, the kinetic law of an elementary process cannot have an arbitrary form. For the general kinetic law (the generalized Mass Action Law), additional conditions are proved. The cell-jump formalism gives an intuitively clear representation of the elementary transport processes and, at the same time, produces kinetic finite elements, a tool for numerical simulation.
\end{abstract}

Key words: diffusion, reaction mechanism, entropy production, detailed balance, complex balance, transport equation

AMS subject classification: $35 \mathrm{~K} 57,35 \mathrm{Q} 82,80 \mathrm{~A} 20,80 \mathrm{~A} 30$

\footnotetext{
${ }^{*}$ Corresponding author. E-mail: ag153@le.ac.uk
} 


\section{Introduction}

\subsection{Linear Diffusion: from Graham and Fick to Einstein, Onsager and Teorell}

\subsubsection{Fick's Law}

The first prominent equation of diffusion is Fick's Law. According to this law, the diffusion flux $J$ is proportional to the antigradient of the concentration $c$ :

$$
J=-D \operatorname{grad} c .
$$

The time derivative of the concentration is the negative of the divergence of the flux:

$$
\frac{\partial c}{\partial t}=-\operatorname{div} J=D \Delta c
$$

where $\Delta$ is the Laplace operator.

This statement is closely related to the Gauss-Ostrogradskii theorem

$$
\iiint_{V}(\operatorname{div} J) \mathrm{d} V=\oiint_{S} J \cdot n \mathrm{~d} S .
$$

The left side is an integral over the volume $V$, the right side is the surface integral over the boundary $S$ of the volume $V, S=\partial V$, and $n$ is the outward pointing unit normal field of $S$. The right-hand side represents the total flow across the boundary "out of the volume $V$ ". The theorem was first discovered by J. L. Lagrange in 1762, then later independently rediscovered by C. F. Gauss in 1813, by G. Green in 1825 and in 1831 by M. V. Ostrogradsky. According to this theorem, the diffusion equation $\partial_{t} c=-\operatorname{div} J$ is just a conservation law: all changes of concentration are caused by the flux only.

In his work on diffusion law, A. Fick used the conservation of matter and the analogy between diffusion and Fouriers law for heat conduction (1822), or Ohms law for electricity (1827). Development of the fundamental law of diffusion was inspired by the Graham's investigations on the diffusion of salts in water [55], in which he studied and compared the diffusibility of different salts.

Before his study of diffusion in liquids, Graham studied diffusion in gases (1833). In 1863, J. C. Maxwell calculated the diffusion coefficients in gases from the Graham data. The results are amazing: "His coefficient of diffusion of $\mathrm{CO}_{2}$ in air is accurate at $\pm 5 \%$. Isn't it extraordinary?" [90].

Maxwell's theory of diffusion was based on gas kinetics and mean fee path estimates.

\subsubsection{Einstein's Mobility}

In his theory of Brownian motion, A. Einstein [24] developed the microscopic theory of the diffusion coefficient for diluted particles in a liquid and compared two processes: the motion of particles in a liquid under a constant external force $K$, and diffusion. For a given $K$, each particle has the 
average velocity $\mathfrak{m} K$ where the coefficient $\mathfrak{m}$ characterizes mobility of particles. (We use $\mathfrak{m}$ for mobility and reserve $\mu$ for chemical potential.) For spherical particles in liquid,

$$
\mathfrak{m}=\frac{1}{6 \pi \eta r},
$$

where $\eta$ is the viscosity of the liquid, $r$ is the radius of particles, and $6 \pi \eta r$ is the Stokes friction force.

This approach results in a very useful relation for the diffusion coefficient:

$$
D=\mathfrak{m} \frac{R T}{N_{A}}=\mathfrak{m} k_{B} T
$$

where $R$ is the gas constant, $N_{A}$ is the Avogadro constant, $k_{B}$ is the Boltzmann constant. The coefficient $\mathfrak{m}$ is called mobility or the Einstein mobility.

Graham's experimental research was extended to solids by W. C. Roberts-Austen [91]. He used Fick's equation to determine the diffusion coefficient [79]. In 1922, S. Dushman and I. Langmuir [22] proposed to use the Arrhenius law for diffusion coefficient:

$$
D=D_{0} \exp (-Q / k T)
$$

where $Q$ is a constant, which we now recognize as the activation Gibbs energy of diffusion $\Delta G$. More precisely, $\Delta G$ includes two terms: $\Delta G=\Delta H-T \Delta S$, where $\Delta H$ is the activation enthalpy and $\Delta S$ is the activation entropy.

They checked this law by their own experiments with the diffusion of thorium through tungsten and found satisfactory agreement. Even better agreement was found with the published results of W. C. Roberts-Austen's experiments.

\subsubsection{Teorell Formula}

The mobility-based approach was further applied by T. Teorell [101]. In 1935, he studied the diffusion of ions through a membrane (Fig. 1). He considered a system of an ideally dilute solution of binary univalent strong electrolysis at the same temperature in water. The boundary is considered to be a membrane with strong a electrolyte in the presence of water. The solutions are assumed to be kept homogeneous on both sides of the membrane up to the boundary by some form of convection. The ionic mobilities within the membrane are assumed constant and may be different, and the membrane is not permeable for water. Heat effects, special membrane effects and chemical reactions are ignored.

He formulated the essence of his approach in the formula:

the flux is equal to mobility $\times$ concentration $\times$ force per gram ion.

This is the so-called Teorell formula.

The force consists of two parts:

1. Diffusion force caused by concentration gradient: $-R T \frac{1}{c} \mathrm{~d} c$. 


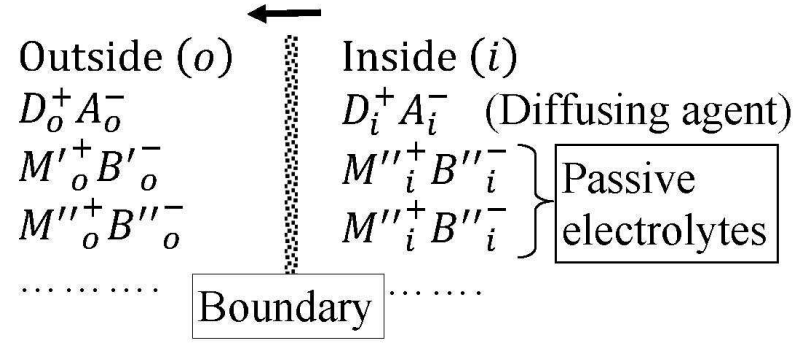

Figure 1: Teorell's model [101]. A system of ideally dilute solutions in water of binary univalent strong electrolytes $D A, M^{\prime} B^{\prime}, M^{\prime \prime} B^{\prime \prime}, \ldots$ is considered. The boundary membrane is permeable for all ions (cations $D, M, \ldots$ and anions $A, B, \ldots$ ) but the movement of water is prevented. The ionic concentrations outside are kept constant. Also $D_{i}^{+}$is maintained constant. Accordingly, $D A$ is a steadily diffusing electrolyte. No other electric field is present besides that due to diffusion potential. No chemical reaction takes place. The steady state of a system of this nature with a steady diffusion is characterized by a constant ratio series: $\frac{M_{i}^{\prime}}{M_{o}^{\prime}}=\frac{M_{i}^{\prime \prime}}{M_{o}^{\prime \prime}}=\cdots=\frac{B_{i}^{\prime}}{B_{o}^{\prime}}=\frac{B_{i}^{\prime \prime}}{M_{o}^{\prime \prime}}=\cdots$.

2. Electrostatic force caused by electric potential gradient: $q \frac{\mathrm{d} \varphi}{\mathrm{d} x}$.

Here $R$ is the gas constant, $T$ is the absolute temperature, $c$ is the concentration, $q$ is the charge and $\varphi$ is the electric potential.

In these notations, the Teorell formula for the flux $J$ is

$$
J=\mathfrak{m} c\left(-\frac{R T}{c} \frac{\mathrm{d} c}{\mathrm{~d} x}+q \frac{\mathrm{d} \varphi}{\mathrm{d} x}\right)
$$

( $\mathfrak{m}$ denotes mobility; here we slightly modernize notations). It may be worthwhile to introduce the reference equilibrium concentrations vector $c^{*}$ and write the diffusion force in the form

$$
-\frac{R T}{c} \frac{\mathrm{d} c}{\mathrm{~d} x}=-R T \frac{\mathrm{d} \ln \left(c / c^{*}\right)}{\mathrm{d} x} .
$$

This expression allowed Teorell to find the concentration jump and the electric potential across the membrane caused by the joint action of diffusion and the electric field, when mobilities of various components are different.

\subsubsection{Onsager's Linear Phenomenology}

In 1931, L. Onsager [83, 84] included diffusion in the general context of linear non-equilibrium thermodynamics. For multi-component diffusion,

$$
J_{i}=\sum_{j} L_{i j} X_{j}
$$

where $J_{i}$ is the flux of the $i$ th component and $X_{j}$ is the $j$ th thermodynamic force (for pure diffusion, this is the space antigradient of the $j$ th chemical potential divided by $T$ ). After linearization near equilibrium, this approach gives for perfect systems (for which the chemical potential is 
$\left.R T \ln \left(c / c^{*}\right)\right)$ :

$$
\begin{aligned}
& X_{j}=-\frac{1}{T} \operatorname{grad} \mu_{j}=-\frac{R}{c_{j}^{*}} \operatorname{grad} c_{j} ; \\
& J_{i}=-\sum_{j} L_{i j} \frac{R}{c_{j}^{*}} \operatorname{grad} c_{j} ; \\
& \frac{\partial c_{i}}{\partial t}=-\operatorname{div} J_{i}=R \sum_{j} L_{i j} \frac{\Delta c_{j}}{c_{j}^{*}},
\end{aligned}
$$

where $c_{j}^{*}$ are equilibrium constants ( $c^{*}$ is the point of linearization), deviations of $c_{j}$ from $c_{j}^{*}$ are assumed to be small, $\Delta$ is the Laplace operator, and $L_{i j}=L_{j i}$ is the matrix of the coefficients. Its symmetry follows from microreversibility.

The system (1.10) has one attractive property. Let us consider this system in a bounded domain $V$ with smooth boundary and with zero fluxes through its boundary: $\left(n, \operatorname{grad} c_{j}\right)=0$ at any point of $\partial V$ at any time ( $n$ is the vector of the outer normal). The positive quadratic functional

$$
S_{2}=\frac{1}{2} \sum_{i} \int_{V} \frac{\left(c_{i}(x)-c_{i}^{*}\right)^{2}}{c_{i}^{*}} \mathrm{~d} x
$$

is the second-order approximation to the relative entropy (or the so-called Kulback-Leubler divergence, see the review paper [47])

$$
S_{K L}=\sum_{i} \int_{V} c(x) \ln \left(\frac{c_{i}(x)}{c_{i}^{*}}\right) \mathrm{d} x .
$$

Let us calculate the time derivative of $S_{2}$ due to the system (1.10). Using the Gauss-Ostrogradskii formula (1.3) we get for the positive semidefinite matrix $L$ :

$$
\frac{\mathrm{d} S_{2}}{\mathrm{~d} t}=-R \int_{V} \sum_{i j} L_{i j}\left(\frac{\nabla c_{i}}{c_{i}^{*}}, \frac{\nabla c_{j}}{c_{j}^{*}}\right) \mathrm{d} x \leq 0,
$$

where $\left(\frac{\nabla c_{i}}{c_{i}^{*}}, \frac{\nabla c_{j}}{c_{j}^{*}}\right)$ is the inner product of the space vectors.

Therefore, $\frac{\mathrm{d} S_{2}}{\mathrm{~d} t} \leq 0$ if the symmetric coefficient matrix $L_{i j}$ is positive semidefinite (this means that for any vector $\xi$ the following inequality holds: $\sum_{i j} L_{i j} \xi_{i} \xi_{j} \geq 0$ ).

The Onsager form of the diffusion equations is correct near the equilibrium but violates the obvious physical requirement: the diffusion flux of the $i$ th component is zero if its concentration has zero value: the flux vanishes with the concentration. The Teorell formula satisfies this requirement. Fick's law also satisfies this requirement in the following sense: if for nonnegative smooth $c(x)$ the concentration vanishes at some points, then at these points the flux vanishes too (because these points are minimizers of concentration and the gradient vanishes there).

For isotropic non-perfect systems we have to use the generalized thermodynamic forces in Onsager's form of the diffusion law:

$$
X_{j}=-\left.\frac{\partial f}{\partial c_{j}}\right|_{c=c^{*}} \operatorname{grad} c_{j},
$$


where $T f(c, T)$ is the free energy density.

Let us denote $\Phi_{i j}=\left(\partial^{2} f / \partial c_{i} \partial c_{j}\right)_{c=c^{*}}$. In this notation,

$$
\begin{aligned}
& X_{j}=-\sum_{k} \Phi_{j k} \operatorname{grad} c_{k} ; J_{i}=\sum_{j} L_{i j} X_{j}=-\sum_{k}\left(\sum_{j} L_{i j} \Phi_{j k}\right) \operatorname{grad} c_{k} ; \\
& \frac{\partial c_{i}}{\partial t}=-\operatorname{div} J_{i}=\sum_{k}\left(\sum_{j} L_{i j} \Phi_{j k}\right) \Delta c_{k} .
\end{aligned}
$$

The quadratic form

$$
F_{2}=\frac{1}{2} \int \sum_{j k} \Phi_{j k}\left(c_{j}-c_{j}^{*}\right)\left(c_{k}-c_{k}^{*}\right) \mathrm{d} x
$$

is positive definite because $F$ is strictly convex. For positive definite $L, F_{2}$ decreases in time due to diffusion. Indeed, in a bounded domain $V$ with a smooth boundary and without fluxes through the boundary we get analogously to (1.13):

$$
\frac{\mathrm{d} F_{2}}{\mathrm{~d} t}=-\int_{V} \sum_{i j}\left(\sum_{k} \Phi_{i k} \nabla c_{k}\right) L_{i j}\left(\sum_{l} \Phi_{j l} \nabla c_{l}\right) \mathrm{d} x \leq 0 .
$$

For non-isotropic diffusion (for example, in crystals), the coefficients $L$ have two pairs of indexes: $L_{i \alpha j \beta}$, where $i, j$ correspond to components and $\alpha, \beta$ correspond to the space coordinates. The forces and fluxes also have these two indexes and

$$
J_{i \alpha}=\sum_{j \beta} L_{i \alpha j \beta} X_{j \beta} .
$$

In all cases, the diffusion equations in Onsager's form do not describe the non-diagonal terms (the influence of gradients $c_{i}$ on fluxes of $c_{j}$ for $i \neq j$ ) properly near zeros of concentrations. These equations are applicable near a reference point $c^{*}>0$ only.

Non-diagonal diffusion must be non-linear. This simple remark is so important that we will explain it in detail. Let diffusion be non-diagonal and linear:

$$
\partial_{t} c_{i}=\sum_{j} D_{i j} \Delta c_{j}
$$

Assume that $D_{12} \neq 0$ and consider the state with $c_{2}=\ldots=c_{n}=0$. At this state,

$$
\partial_{t} c_{2}=D_{12} \Delta c_{1}
$$

If $D_{12} \Delta c_{1}(x)<0$ at some points then $c_{2}(x)$ becomes negative at these point in a short time. Therefore, linear non-diagonal diffusion does not preserve positivity of concentrations. 


\subsection{Mechanisms of Nonlinear Diffusion}

\subsubsection{Jumps on the Surface}

In 1980, A.N, Gorban, V.I. Bykov and G.S. Yablonskii [45] proposed a model for diffusion in monolayers of reagents on the surface of a catalyst, which is based on the jumps of the reagents on the nearest free places. This model was used for $\mathrm{CO}$ on Pt oxidation under low gas pressure.

The system includes several reagents $A_{1}, A_{2}, \ldots A_{n}$ on the surface. Their surface concentrations are $c_{1}, c_{2}, \ldots c_{n}$. The surface is a lattice of the adsorbtion places. Each reagent molecule fills a place on the surface. Some of the places are free. We use $Z=A_{0}$ for a free place and the concentration is $z=c_{0}$. The sum of all $c_{i}$ (including free places) is constant:

$$
\sum_{i=0}^{n} c_{i}=b=\mathrm{const} .
$$

The jump model gives for the diffusion flux of $A_{i}(i=1, \ldots, n)$ :

$$
J_{i}=-D_{i}\left[z \nabla c_{i}-c_{i} \nabla z\right]
$$

Therefore, the corresponding diffusion equation is:

$$
\frac{\partial c_{i}}{\partial t}=-\operatorname{div} J_{i}=D_{i}\left[z \Delta c_{i}-c_{i} \Delta z\right]
$$

Due to the conservation law,

$$
z=b-\sum_{i=1}^{n} c_{i},
$$

and we have the system of $n$ diffusion equations:

$$
\begin{aligned}
& J_{i}=-D_{i}\left[\left(b-\sum_{i=1}^{n} c_{i}\right) \nabla c_{i}+c_{i} \nabla\left(\sum_{i=1}^{n} c_{i}\right)\right] \\
& \frac{\partial c_{i}}{\partial t}=D_{i}\left[\left(b-\sum_{i=1}^{n} c_{i}\right) \Delta c_{i}+c_{i} \Delta\left(\sum_{i=1}^{n} c_{i}\right)\right] .
\end{aligned}
$$

It is straightforward to check that when $c_{i} \geq 0$ for all $x$ then $\partial_{t} c_{i} \geq 0$ for $c_{i}=0$. This is a necessary condition for preservation of positivity.

If we assume that all particles can exchange their positions with their closest neighbors then a simple generalization of (1.17), (1.19) appears:

$$
\begin{aligned}
& J_{i}=-\sum_{j} D_{i j}\left[c_{j} \nabla c_{i}-c_{i} \nabla c_{j}\right] \\
& \frac{\partial c_{i}}{\partial t}=\sum_{j} D_{i j}\left[c_{j} \Delta c_{i}-c_{i} \Delta c_{j}\right],
\end{aligned}
$$


where $D_{i j}=D_{j i} \geq 0$ is a symmetric matrix of coefficients which characterize the intensities of jumps.

The entropic Lyapunov functional for (1.19), (1.20) has a simple traditional form of perfect relative entropy: for any reference vector of concentrations $c^{*}\left(c_{i}^{*} \geq 0\right)$

$$
S_{K L}=\int \sum_{i} c_{i} \ln \left(\frac{c_{i}}{c_{i}^{*}}\right) \mathrm{d} x
$$

Remark: the free place entropy should be obligatorily included into $S_{K L}$.

Simple algebra gives that in a bounded domain $V$ with a smooth boundary and without fluxes through boundaries

$$
\frac{\mathrm{d} S_{K L}}{\mathrm{~d} t}=-\sum_{i j} D_{i j} \int_{V}\left(\frac{c_{i}}{c_{j}} \nabla c_{j}-\frac{c_{j}}{c_{i}} \nabla c_{i}\right)^{2} \mathrm{~d} x \leq 0 .
$$

This inequality provides the Lyapunov stability of diffusion.

It is worth mentioning that the thermodynamic inequality (1.22) requires only the non-negativity of coefficients $D_{i j}$ and does not imply any requirements on the matrix $D$ as a whole (like positive definiteness). Another form of the thermodynamic inequality makes the formula for the entropy production more transparent:

$$
\frac{\mathrm{d} S_{K L}}{\mathrm{~d} t}=-\sum_{i j} D_{i j} \int_{V}\left(c_{i} \nabla \ln \left(\frac{c_{j}}{c_{j}^{*}}\right)-c_{j} \nabla \ln \left(\frac{c_{i}}{c_{i}^{*}}\right)\right)^{2} \mathrm{~d} x \leq 0 .
$$

This system of models was further developed by A.N. Gorban and H.P. Sargsyan [53] and published in a book [46].

\subsubsection{Diffusion in Solids as Reaction: from Frenkel to Eyring}

The physical idea of the quasi-chemical representation of diffusion in solids belongs to Yakov Frenkel $[36,37]$. He introduced both the vacancy and the interstitial mechanisms of diffusion and found some rate constants from experimental data.

Thirty years later, F. C. Frank and D. Turnbull developed the Frenkel theory further [34]. They studied the diffusion of copper in germanium. This diffusivity is very rapid. They proposed that the copper could be dissolved in two states, interstitial and substitutional. For the interstitial state the solubility of copper is two orders of magnitude less and the diffusivity many orders of magnitude greater than in the substitutional state. The conversion of these states is effected by lattice vacancies.

The quasi-chemical theory of diffusion and viscosity was developed also by H. Eyring with co-authors [66]. Eyring developed the theory of absolute reaction rates for chemical reactions in gases [28] and in condensed phase [116] and then applied these ideas to transport phenomena.

In this theory, the transport process is represented by an ensemble of elementary events. Each elementary event is represented by the creation or disintegration of an activated complex. The rate 
of the elementary process is given by the concentration of activated complexes, multiplied by the rate at which they decompose.

The main constructive hypothesis is that it is possible to calculate the concentration of activated complexes by equilibrium statistical thermodynamics: the complex concentration is in quasiequilibrium with the stable components. Each complex has its "internal translational" degree of freedom. On the surface of potential energy this corresponds to the "reaction path". Complexes move along this path. The velocity of this motion is assumed to be just a thermal velocity and is proportional to $\sqrt{T}$.

The additional reaction path degree of freedom has its own kinetic energy and, therefore, increases the complex heat capacity. We have to take this into account in the calculation of the equilibrium constant.

Collective models of diffusion were proposed too. One of the earliest collective model is the Z. Jeffries "ring mechanism" with 4 or more atoms. More on the history of solid-state diffusion is presented in the review [104] and in a modern textbook [78].

On the surface, there are various mechanisms for collective diffusion [88] as well. Elementary events for these mechanisms involve many atoms simultaneously. A dynamic description of nonlinear multicomponent diffusion requires a unified framework that should satisfy basic physical principles.

\subsubsection{Ginzburg-Landau Free energy and Cahn-Hilliard equation}

The processes of phase separation has remained for a long time an important source of problems and ideas for the theory of nonlinear diffusion. The analogue for Fick's equation is the CahnHilliard equation [12].

The Cahn-Hilliard equation in its simplest form has the standard Onsager form, the flux is proportional to the force, the force is the gradient of the chemical potential:

$$
J=-D \nabla \mu, \frac{\partial c}{\partial t}=-\operatorname{div} J=D \Delta \mu .
$$

If we compare this equation to the Teorell formula then we immediately find the missed factor $c$ (concentration). We will return to the problem of the proper prefactor in the Cahn-Hilliard equations later. The main specificity of the Cahn-Hilliard equations is the form of the free energy and the chemical potential $[12,11]$ (the Ginzburg-Landau form):

$$
f=f^{c}(c)+\gamma(\nabla c)^{2}, F=\int f \mathrm{~d} x, \mu=\frac{\partial f^{c}(c)}{\partial c}-\gamma \Delta c .
$$

The term $\gamma(\nabla c)^{2}$ in the free energy penalizes ovr sharp gradients and, in particular, models the interface energy.

According to (1.24) and (1.25), the Cahn-Hilliard equation reads

$$
J=-D \nabla\left(\frac{\partial f^{c}(c)}{\partial c}-\gamma \Delta c\right), \frac{\partial c}{\partial t}=D \Delta\left(\frac{\partial f^{c}(c)}{\partial c}-\gamma \Delta c\right)
$$


If the chemical part of the free energy, $f^{c}(c)$ is not convex then phase separation is possible. If at the point $c$ this function is concave (spinodal) then without the term with $\Delta^{2}$ the constant solution $c(x)=c$ for the diffusion equation becomes unstable to any perturbation (negative diffusion coefficient). The term $-\gamma \Delta^{2} c$ in the right hand part of the Cahn-Hilliard equation regularizes solutions and the existence theorem was proved for the initial-boundary value problem given smooth initial data [26]. The proof relies essentially on the existence of a Lyapunov functional $F$ (1.25).

The time derivative of $F$ in a domain $V$ with a smooth boundary and without external fluxes $((J, n)=0$ on the boundary, where $n$ is the vector of outer normal to the boundary) is

$$
\begin{aligned}
\frac{\mathrm{d} F}{\mathrm{~d} t} & =\int_{V} \mu \partial_{t} c \mathrm{~d} x=\int_{V} \mu \operatorname{div} J \mathrm{~d} x \\
& =-\int_{V}(\nabla \mu, J) \mathrm{d} x=-D \int_{V}(\nabla \mu)^{2} \mathrm{~d} x \leq 0 .
\end{aligned}
$$

If we correct the Cahn-Hilliard equation by the "Teorell" factor $c$ then the dissipation inequality $\dot{F} \leq 0$ (1.27) persists: Due to the Teorell formula

$$
\begin{aligned}
& J=-\mathfrak{m} c \nabla \mu=-\mathfrak{m} c \nabla\left(\frac{\partial f^{c}(c)}{\partial c}-\gamma \Delta c\right), \\
& \frac{\partial c}{\partial t}=-\operatorname{div} J=\mathfrak{m} \operatorname{div}\left(c \operatorname{grad}\left(\frac{\partial f^{c}(c)}{\partial c}-\gamma \Delta c\right)\right) .
\end{aligned}
$$

Here, $\mathfrak{m}$ is the Einstein mobility. This equation should be called "The Cahn-Hilliard-Teorell" equation. In accordance with (1.28),

$$
\begin{aligned}
\frac{\mathrm{d} F}{\mathrm{~d} t} & =\int_{V} \mu \partial_{t} c \mathrm{~d} x=\int_{V} \mu \operatorname{div} J \mathrm{~d} x \\
& =-\int_{V}(\nabla \mu, J) \mathrm{d} x=-\mathfrak{m} \int_{V} c(\nabla \mu)^{2} \mathrm{~d} x \leq 0 .
\end{aligned}
$$

This dissipation inequality allows us to transfer all the results about solutions of the Cahn-Hilliard equation to the Cahn-Hilliard-Teorell equation.

\subsubsection{Teorell Formula for Non-perfect Systems}

It seems very natural that the flux is proportional to the concentration of particles: the average velocity is proportional to the force and the total flux is the product of the average velocity and the amount of moving particles. This could be proved in the framework of non-equilibrium thermodynamics and the theory of absolute reaction rates when the concentration of moving particles is small. In perfect gases or in dilute solutions the chemical potential is

$$
\mu=R T \ln c+\mu_{0},
$$

where $\mu_{0}$ does not depend on $c$ (it is a function of $T$ and the state of the environment). In this case, we neglect the interaction between moving particles and use the Teorell formula (exactly as Einstein did in his theory of Brownian motion 30 years before Teorell [24]). 
When the concentration of moving particles $c$ is not small enough then the formula for perfect chemical potential is no longer valid and in front of $c$ in the flux a special activity coefficient $\alpha$ appears.

Such coefficients were introduced for diffusion by Eyring at al in 1941 [66] and were used systematically for the theory of nonlinear diffusion by Gorban at al in the 1980-1986 [46]. Roughly speaking, the activity

$$
a=\exp \left(\frac{\mu}{R T}\right)
$$

should substitute the concentration $c$ in the Teorell formula with the proper renormalization of the mobility coefficients:

$$
J=\mathfrak{m}^{\prime} a(-\nabla \mu+(\text { external force per gram particle })) .
$$

The renormalized coefficient $\mathfrak{m}^{\prime}$ is defined by the condition: $\left(\mathfrak{m}^{\prime} a\right) /(\mathfrak{m} c) \rightarrow 1$ for $c \rightarrow 0, \nabla c \rightarrow 0$. This means that

$$
\mathfrak{m}^{\prime}=\mathfrak{m} \exp \left(-\frac{\mu_{0}}{R T}\right) \text { where } \mu_{0}=\lim _{c \rightarrow 0}(\mu-R T \ln c),
$$

or we can write the Teorell formula for non-perfect systems using the usual Einstein mobility $\mathfrak{m}$ defined for small concentrations and this standard value of chemical potential, $\mu_{0}$ :

$$
J=\mathfrak{m} \exp \left(\frac{\mu-\mu_{0}}{R T}\right)(-\nabla \mu+(\text { external force per gram particle })) .
$$

This formula is the main analogue of Fick's law for monomolecular diffusion in non-perfect media.

For the Cahn-Hilliard-Teorell equation (1.28), the Teorell formula for non-perfect systems (1.30) significantly changes the diffusion coefficient: the regularizing gradient term should appear in the activity coefficient.

More details about activity coefficients in thermodynamics can be found, for example, in Chapter 9 of the classical book [21].

In the phase separation problem, the components are definitely non-perfect and the further correction of the Cahn-Hilliard-Teorell equation by the activity coefficients is necessary.

The problem of the extension of the Cahn-Hilliard approach to multicomponent diffusion was discussed by various authors [76, 4]. Elastic forces and plasticity are also taken into account $[4,35]$. Nevertheless, the problem of the proper equations of multicomponent nonlinear diffusion in highly non-homogeneous condensed phases is still open. From our point of view, there is no single "proper model" and the variety of possible models is very rich. In our work, we attempt to formulate the proper language for the description of the universe of these models similarly to chemical kinetics models.

\subsection{Main Ideas}

\subsubsection{Mechanisms as Collections of Elementary Processes}

A complex process can be disassembled into several elementary processes. The dependence of the process rate (the flux) on the state (concentrations, chemical potentials and their gradients) 
is simple for elementary processes. The model of the whole process is assembled from these elementary "details".

This idea was developed in chemical kinetics. In 1862-1867, Guldberg and Waage proposed the mass action law for equilibrium. In 1879 they developed the mass action law for dynamics. This idea was developed further by many researchers and after several dozen years it was transformed into a technology for the representation of complex processes: A complex reaction is represented as an ensemble of elementary reactions. The reaction rate has a simple monomial dependence on concentration.

Van't Hoff [107] called the reactions that satisfy the mass action law "normal transformations" and found that "normal transformations take place very rarely". Now we can say that most reactions are complex and the interaction of several elementary reactions causes non-trivial complex ("abnormal") behavior.

Van't Hoff did not study complex reactions by disassembling them into several elementary reactions. Therefore, he was disappointed with the mass action law and finally wrote: "As a theoretical foundation I did not accept the concept of mass action, I had to abandon this concept in the course of my experiments...". ("J'ai adopté pour la théorie, non la notion des masses actives, notion que j' ai dû abandonner dans le cours de mes expériences, ...” [107], p. 7.)

The set of elementary reactions which constitute a complex reactions is called the reaction mechanism. A mechanic analogue is obvious: the elementary reactions are the details of the mechanism that represents the complex reaction. This notion was, finally, introduced into chemistry in the 20th century due to the efforts of M. Bodenstein.

M. Boudart described the "century of Bodenstein" in his paper [5]: "First came the data, then the rate equation, and finally the fitting of the data into the rate equation by means of a hypothesized mechanism with rate constants chosen for the best fit."

The great success of this approach was the theory of chain reactions. N.N. Semenov was awarded the Nobel prize for this theory [94]. The modern theory of complex chemical reactions is based on the idea of the detailed reaction mechanism and the simple kinetic law of elementary reactions [117].

Many authors proposed various particular mechanisms of nonlinear diffusion. One of our goals in this work is to repeat the way of chemical kinetics in application to multicomponent diffusion and to create a comprehensive theory of the mechanisms of diffusion.

\subsubsection{Discrete Kinetic Models and Lattice Automata}

In the 1940s, S. Ulam and J. von Neumann proposed networks of interconnected finite-state automata for the modeling of complex systems. In the first period of study, research was focused on the abilities of these networks and, in particular, on the ability of self-reproduction [111].

The behavior of these cellular automata is so variable and surprising and its complexity is so high that Ulam proposed the idea of the computational experiment: we should regard our invention as a new sort of reality and study it by the experimental approach, as physics or chemistry does.

Cellular automata were invented as an intellectual journey but soon were recognized as efficient tools for modeling [103]. 
Feynman's attention to automata with local interactions as a tool for simulating physics, attracted much attention to this area: "Therefore my question is, can physics be simulated by a universal computer? I would like to have the elements of this computer locally interconnected, and therefore sort of think about cellular automata as an example (but I don't want to force it). But I do want something involved with the locality of interaction. I would not like to think of a very enormous computer with arbitrary interconnections throughout the entire thing" [31].

The idea of modeling the natural world in terms of the behavior of sets of rules that can be embodied in simple automata with local interactions is now an important part of science. Sometimes it is called "the new science" to distinguish this approach from classical modeling by equations [115].

For the modeling of transport processes the lattice gas automata $[114,16]$ were invented and the lattice Boltzmann methods [98] became very popular: they are flexible and efficient. At the same time, the lattice Boltzmann methods are very simple for programming and parallelization.

The essence of the lattice Boltzmann methods was formulated by S. Succi in the following maxim: "Nonlinearity is local, non-locality is linear" [100]. We should even strengthen this statement. Non-locality (a) is linear; (b) is exactly and explicitly solvable for all time steps; (c) space discretization is an exact operation.

The lattice Boltzmann method is a discrete velocity method. The finite set of velocity vectors $\left\{v_{i}\right\}(i=1, \ldots m)$ is selected, and a fluid is described by associating, with each velocity $v_{i}$, a single-particle distribution function $f_{i}=f_{i}(x, t)$ which is evolved by advection and interaction (collision) on a fixed computational lattice. The values $f_{i}$ are named populations. If we look at all lattice Boltzmann models, one finds that there are two steps: free flight for time $\delta t$ and a local collision operation.

The free flight transformation for continuous space is

$$
f_{i}(x, t+\delta t)=f_{i}\left(x-v_{i} \delta t, t\right) .
$$

After the free flight step the collision step follows:

$$
f_{i}(x) \mapsto F_{i}\left(\left\{f_{j}(x)\right\}\right)
$$

or in the vector form

$$
f(x) \mapsto F(f(x)) .
$$

Here, the collision operator $F$ is the set of functions $F_{i}\left(\left\{f_{j}\right\}\right)(i=1, \ldots m)$. Each function $F_{i}$ depends on all $f_{j}(j=1, \ldots m)$ : new values of the populations $f_{i}$ at a point $x$ are known functions of all previous population values at the same point.

The lattice Boltzmann chain "free flight $\rightarrow$ collision $\rightarrow$ free flight $\rightarrow$ collision.. " can be exactly restricted onto any space lattice which is invariant with respect to space shifts of the vectors $v_{i} \delta t(i=1, \ldots, m)$. Indeed, free flight transforms the population values at sites of the lattice into the population values at sites of the same lattice. The collision operator (1.31) acts pointwise at each lattice site separately. Much effort has been applied to answer the questions: "how does the lattice Boltzmann chain approximate the transport equation for the moments $M$ ?", and "how does 
one construct the lattice Boltzmann model for a given macroscopic transport phenomenon?" (a review is presented in the book [98]).

The lattice Boltzmann models should describe the macroscopic dynamic, i.e., the dynamic of macroscopic variables. The macroscopic variables $M_{\ell}(x)$ are some linear functions of the population values at the same point: $M_{\ell}(x)=\sum_{i} m_{\ell i} f_{i}(x)$, or in the vector form, $M(x)=m(f(x))$. The macroscopic variables are invariants of collisions:

$$
\sum_{i} m_{\ell i} f_{i}=\sum_{i} m_{\ell i} F_{i}\left(\left\{f_{j}\right\}\right) \quad(\text { or } m(f)=m(F(f))) .
$$

The standard example of the macroscopic variables are hydrodynamic fields (density-velocityenergy density): $\{n, u, E\}(x):=\sum_{i}\left\{1, v_{i}, v_{i}^{2} / 2\right\} f_{i}(x)$. But this is not an obligatory choice. On the other hand, the athermal lattice Boltzmann models with a shortened list of macroscopic variables $\{n, n u\}$ are very popular.

The quasiequilibrium is the positive fixed point of the collision operator for given macroscopic variables $M$. We assume that this point exists, is unique and depends smoothly on $M$. For the quasiequilibrium population vector for given $M$ we use the notation $f_{M}^{*}$, or simply $f^{*}$, if the correspondent value of $M$ is obvious. We use $\Pi^{*}$ to denote the equilibration projection operation of a distribution $f$ into the corresponding quasiequilibrium state:

$$
\Pi^{*}(f)=f_{m(f)}^{*} .
$$

Usually, collision operators are taken in the form:

$$
F(f):=\Pi^{*}(f)+A\left(\Pi^{*}(f)-f\right),
$$

where $A$ is a linear operator, whose spectrum belongs to the interior of the unit circle. A special case of (1.32) is very popular, the lattice Bhatnagar-Gross-Krook (LBGK) model:

$$
F(f):=f+\omega\left(\Pi^{*}(f)-f\right) .
$$

In this brief introduction of LBM we follow the paper [8].

The simplest LBGK realization of Fick's law (in 1D) gives the following system. The discrete velocity set includes two elements only, $v$ and $-v$. The time step is $\tau$ the correspondent grid step is $h=v \tau$. The microscopic variables, the populations, are: $f^{-}$for velocity $-v$ and $f^{+}$for $v$. The macroscopic variable, the density, is $\rho=f^{-}+f^{+}$. The corresponding equilibrium is $\Pi^{*}(f)=f^{*}$ :

$$
f^{*+}=f^{*-}=\frac{f^{-}+f^{+}}{2}
$$

For the non-negative populations, the equilibrium distribution is the maximizer of the entropy $S=-f^{-} \ln f^{-}+f^{+} \ln f^{+}$under a given value of the macroscopic variable $\rho$.

Let us take the LBGK collisions (1.33) with $\omega=1$, i.e.

$$
F(f)^{ \pm}=\Pi^{*}(f)^{ \pm}=\frac{f^{-}+f^{+}}{2} .
$$


This particular case of the LBGK collision integral is an example of the so-called Ehrenfests' coarse-graining. The idea of artificial partial equilibration steps was proposed by T. and P. Ehrenfest for the foundation of statistical physics [23] and further developed to a general formalism of nonequlibrium thermodynamics [48, 18, 49].

A review and comparative analysis of different approaches to coarse-graining was published in [44].

The LBGK chain for the collision integral (1.34) has a very simple form:

$$
\begin{aligned}
& f^{+}(n h,(m+1) \tau)=f^{-}(n h,(m+1) \tau) \\
& =\frac{f^{+}((n-1) h, m \tau)+f^{-}((n+1) h, m \tau)}{2} .
\end{aligned}
$$

Therefore, for the density $\rho$ we get

$$
\rho(n h,(m+1) \tau)=\frac{\rho((n-1) h, m \tau)+\rho((n+1) h, m \tau)}{2} .
$$

This appears to be the one of the most common explicit finite difference methods for Fick's diffusion equation. The diffusion coefficient is $D=h^{2} /(2 \tau)=v^{2} \tau / 2$ and depends explicitly on the lattice parameters. We can decouple $D$ and the lattice parameters if we use $\omega \in[1,2]$ in the LBGK collision integral (1.33). This lattice-gas scheme does not coincide with any of the finite difference schemes. Nevertheless, it also models diffusion and, to the first order in $\tau, D=v^{2} \tau \frac{2-\omega}{2 \omega}[98,44]$.

Now, the area of applications of the cellular and lattice Boltzmann automata is very wide and, in addition to classical fluid dynamics, includes many areas of chemistry [65], models of phase separation [92], dynamics of macromolecules and many other topics.

We use cellular automata and lattice models of nonlinear multicomponent diffusion for two purposes:

- As a tool for model creation (after that, this model could be translated into other languages, such as partial differential equations (PDE);

- As a tool for numerical simulation without the stage of PDE model.

Elliott and Stuart [27] used the cell model of diffusion to study semilinear parabolic equations. They proved the existence of absorbing sets, bounded independently of the mesh size for discrete models. Discrete Lyapunov functions were constructed. We use the special quasichemical approach for the generation of the the cell models [46] that allowed us to construct the Lyapunov functions for semi-discrete systems and to prove stabilization of the solution in space and time under proper conditions.

Let us consider our space divided into cells, a system represented as a chain of cells of homogeneous composition and elementary transfer processes between them. It is sufficient for our purposes to discuss two sells (Fig 2). Let us numerate these cells by the Roman numbers I and II and mark all the components and quantities related to them by the upper index I or II, correspondingly. The lists of the components for cells are different just by the upper index: $A_{1}^{\mathrm{I}}, \ldots A_{n}^{\mathrm{I}}$, $A_{1}^{\mathrm{II}}, \ldots A_{n}^{\mathrm{II}}$. 


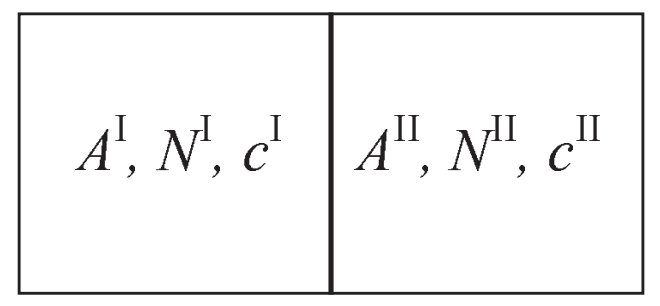

Figure 2: Cell Jump Model

The mechanism of diffusion is defined as a list of elementary transitions between cells described by their stoichiometric equation. Since diffusion is a sort of jumping reaction on the border, for these jumps the stoichiometric equation is written as,

$$
\sum_{i} \alpha_{r i}^{\mathrm{I}} A_{i}^{\mathrm{I}}+\sum_{i} \alpha_{r i}^{\mathrm{II}} A_{i}^{\mathrm{II}} \rightarrow \sum_{i} \beta_{r i}^{\mathrm{I}} A_{i}^{\mathrm{I}}+\sum_{i} \beta_{r i}^{\mathrm{II}} A_{i}^{\mathrm{II}},
$$

where $r$ is the number of processes, $\alpha_{r i}^{\mathrm{I}, \mathrm{II}}$, and $\beta_{r i}^{\mathrm{I}, \mathrm{II}}$, are the stoichiometric coefficients which indicate the number of particles in cells involved in the process. The direction of changes in the elementary event (1.37) is defined by two stoichiometric vectors

$$
\gamma_{r i}^{\mathrm{I}}=\beta_{r i}^{\mathrm{I}}-\alpha_{r i}^{\mathrm{I}} ; \gamma_{r i}^{\mathrm{II}}=\beta_{r i}^{\mathrm{II}}-\alpha_{r i}^{\mathrm{II}}
$$

Examples of elementary acts are presented in Fig. 3.

Elementary events (1.37) should not include reactions. Therefore, for each $i$, the amount of $A_{i}$ in the system $\left(A_{i}^{\mathrm{I}}+A_{i}^{\mathrm{II}}\right)$ should not change. This means exactly that for all $i, r$

$$
\gamma_{r i}^{\mathrm{I}}=-\gamma_{r i}^{\mathrm{II}}
$$

Let us use the notation

$$
\gamma_{r i}=\gamma_{r i}^{\mathrm{I}}=-\gamma_{r i}^{\mathrm{II}} .
$$

The composition of each cell is a vector $N^{\mathrm{I}, \mathrm{II}}$. The components of this vector, $N_{i}^{\mathrm{I}, \mathrm{II}}$ are the amounts of $A_{i}$ in the correspondent cell. We describe the dynamics of the compositions of two cells by the equations:

$$
\frac{d N^{\mathrm{I}}}{d t}=-\frac{d N^{\mathrm{II}}}{d t}=S \sum_{r} \gamma_{r} w_{r},
$$

where $S$ is the area of the boundary between two cells and $w_{r}$ is the rate of the process. For many cells the equations are the same, but with more pairs of cells interacting, and therefore there are more terms.

The rates are intensive variables and should be defined as functions of concentrations or chemical potentials. The crucial question is: how to describe function $w_{r}\left(c^{\mathrm{I}}, c^{\mathrm{II}}\right)$, where $c^{\mathrm{I}, \mathrm{II}}$ are concentrations components in cells.

The real physics of diffusion may be more complicated. For example, the intensity of jumps and the reaction rate $w_{r}\left(c^{\mathrm{I}}, c^{\mathrm{II}}\right)$ may depend not only on $\left(c^{\mathrm{I}}, c^{\mathrm{II}}\right)$ but on the surrounding. For 

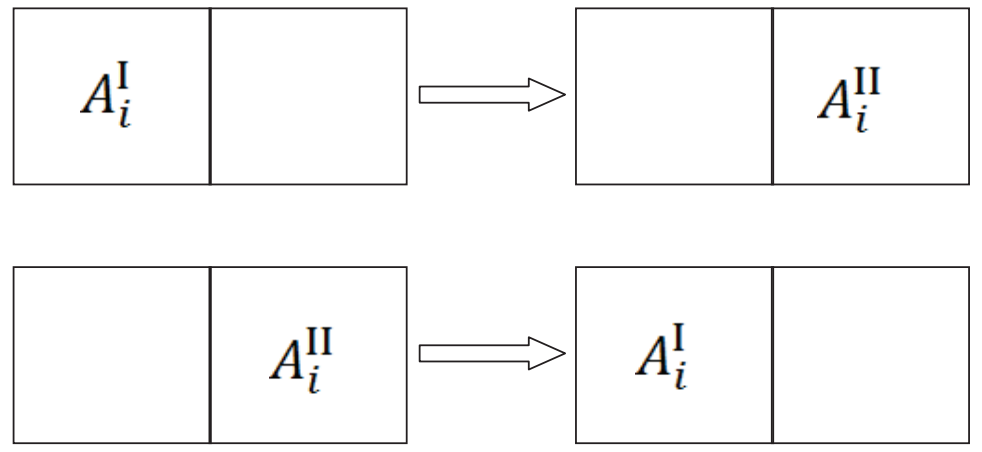

(a) Simple diffusion: a particle from the cell I jumps into the cell II and inverse
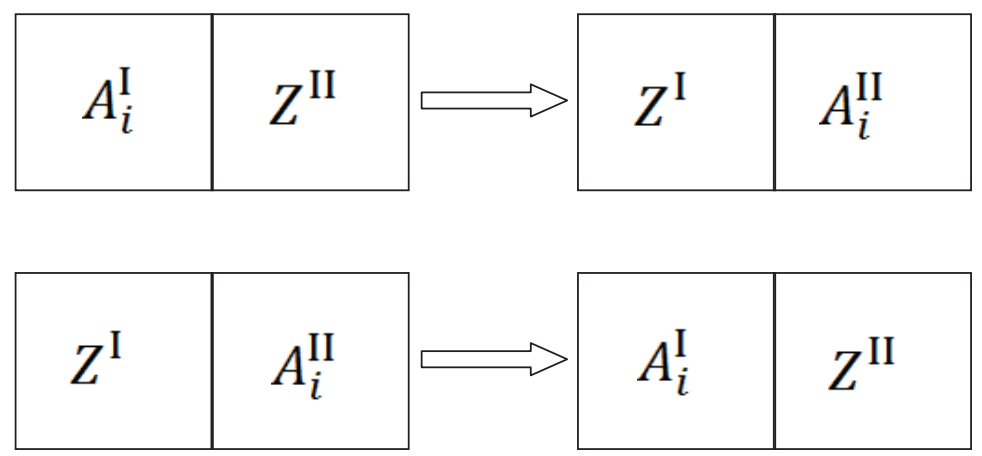

(b) Jumps to free places: a particle from the cell I jumps to the free place in cell II and inverse
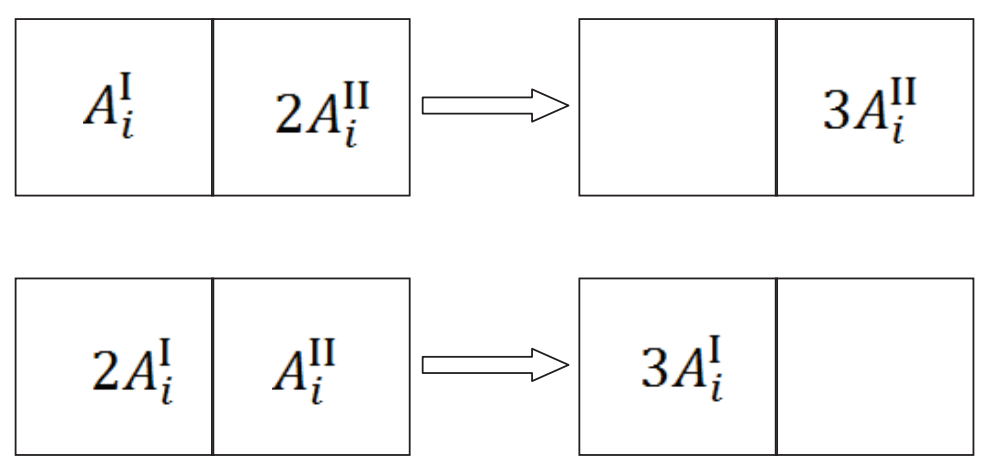

(c) Jumps with clustering: two particle attract the third one

Figure 3: Elementary acts of diffusion, examples. 


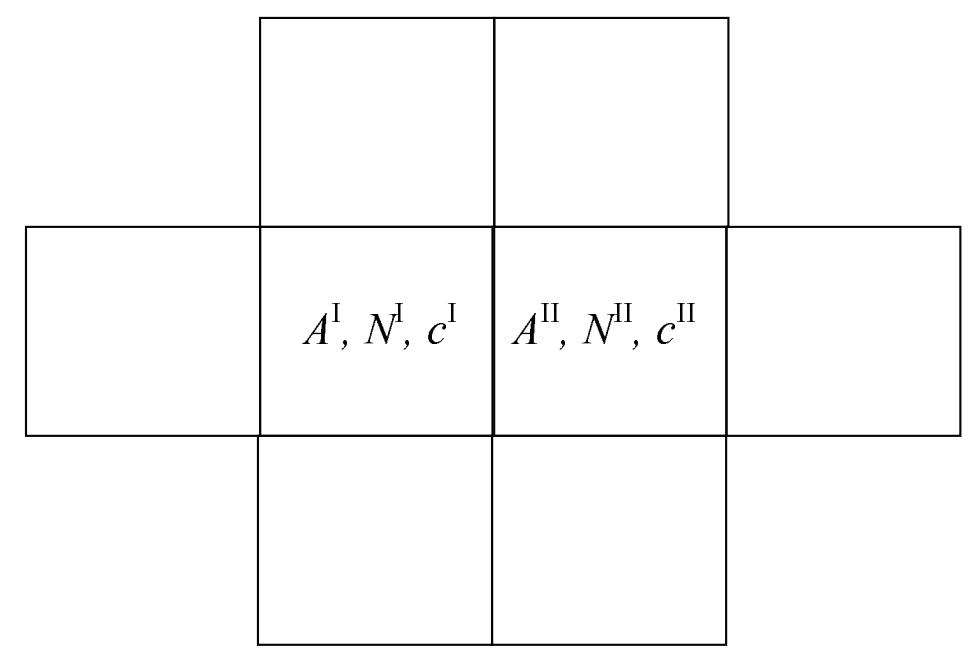

Figure 4: Cell Jump Model with first surroundings.

example, direct simulation of the jumps on the surface [9] demonstrates that the influence of the surrounding is crucial for structures and critical effects on the surface.

For each process (1.37) there is the space-inverted process that is defined just by changing I to II and vice versa. We mark the quantities for the space-inverted processes by '. For example, $\gamma^{\prime}=-\gamma$. The detailed space-inversion symmetry requires that the rate functions for them should differ just by the transposition of the vectors of variables, $c^{\mathrm{I}}, c^{\mathrm{II}}$ :

$$
w_{r}^{\prime}\left(c^{\mathrm{I}}, c^{\mathrm{II}}\right)=w_{r}\left(c^{\mathrm{II}}, c^{\mathrm{I}}\right) .
$$

This requirement of detailed space symmetry allows us, in particular, to exclude various types of advection and transport driven by external force. Diffusion, by its definition, is driven by the gradients of the concentrations (or, in the thermodynamical approach, by the gradients of the chemical potentials). This is not the only way to formulate of pure diffusion equations without advection. Another possibility gives us, for example, the diffusion systems with complex balance (Section 2.5.).

There are three ways to define the rate functions: from a phenomenological law (like the mass action law), from thermodynamics (like the generalized mass action law) or by direct stochastic simulation of particles jumps in cells (like in the Gillespie approach [40, 41]).

In our research, we focus on the first two approaches. Therefore, we consider our lattice model as a semi-discrete model (discrete in space and continuous in time). For this semi-discrete model, the system of kinetic equations (1.38) describes diffusion. The continuous limit of these equations gives us the diffusion PDE. The discrete scheme by itself can serve as a computational model.

A couple of simple examples can clarify our approach:

- Simple diffusion (Fig. 3(a)), $A_{i}^{\mathrm{I}} \rightarrow A_{i}^{\mathrm{II}}$ and $A_{i}^{\mathrm{II}} \rightarrow A_{i}^{\mathrm{I}}$ with the same rate constants. Particles jump into the neighbor cells. For perfect mixtures, $w_{r}=k c_{i}^{\mathrm{I}}, w_{r}^{\prime}=k c_{i}^{\mathrm{II}}$ and in the contin- 
uous limit we get Fick's law (1.2) as the first Taylor approximation. In this approximation, $D=k l$ where $l$ is the cell size.

- Jumps to free places (Fig. 3(b)), $A_{i}^{\mathrm{I}}+Z^{\mathrm{II}} \rightarrow A_{i}^{\mathrm{II}}+Z^{\mathrm{I}}$ and $A_{i}^{\mathrm{II}}+Z^{\mathrm{I}} \rightarrow A_{i}^{\mathrm{I}}+Z^{\mathrm{II}}$. According to the mass action law, $w_{r}\left(c^{\mathrm{I}}, c^{\mathrm{II}}\right)=k c_{i}^{\mathrm{I}} z^{\mathrm{II}}, w_{r}^{\prime}\left(c^{\mathrm{I}}, c^{\mathrm{II}}\right)=k c_{i}^{\mathrm{II}} z^{\mathrm{I}}$, where $z$ is the concentration of free places. In the first Taylor approximation, $J=-k l\left(z \nabla c_{i}-c_{i} \nabla z\right)$ and we get the model (1.17), (1.19).

To get the continuous limit, we take $c^{\mathrm{I}}=c(x), c^{\mathrm{II}}=c(x+l)$ and use the Taylor expansion: $c(x+l)=c(x)+l \partial_{x} c+o(l)$. If we the consider a sequence of cell representations of diffusion with various $l$ then, for the invariance of the first order, the scaling rule should be implemented: $D=k l$ does not change with the change of size, therefore, the rate constant $k$ depends on $l$ : $k=D / l$.

It is not always possible to keep to first order only. If this approach gives a negative diffusion coefficient then for regularity we have to keep the higher derivatives. For example, let us take the diffusion mechanism with attraction:

$$
A_{i}^{\mathrm{I}}+2 A_{i}^{\mathrm{II}} \rightarrow 3 A_{i}^{\mathrm{II}} .
$$

The space-inverted process in this case does not coincide with the inverse one. If we change the upper indexes (I to II and II to I) then we obtain

$$
2 A_{i}^{\mathrm{I}}+A_{i}^{\mathrm{II}} \rightarrow 3 A_{i}^{\mathrm{I}} .
$$

This mechanism means that 2 particles attract the third one. This mechanism is represented in Fig. 3(c).

The reaction rates are:

$$
w_{r}=k_{r} c_{i}^{\mathrm{I}}\left(c_{i}^{\mathrm{II}}\right)^{2}, w_{r}^{\prime}=k_{r}\left(c_{i}^{\mathrm{I}}\right)^{2} c_{i}^{\mathrm{II}} .
$$

The flux of $A_{i}$ from the first cell to the second one is

$$
J=w_{r}-w_{r}^{\prime}=k_{r} c_{i}^{\mathrm{I}} c_{i}^{\mathrm{II}}\left(c_{i}^{\mathrm{II}}-c_{i}^{\mathrm{I}}\right) .
$$

Therefore, to first order we have

$$
J=k l c^{2} \nabla c=\frac{1}{3} k l \nabla c^{3}
$$

the sign is opposite to standard diffusion. This flux goes in the direction of gradients. The diffusion equation is

$$
\frac{\partial c}{\partial t}=-k l \operatorname{div}\left(c^{2} \nabla c\right)=-k l \frac{1}{3} \Delta c^{3}
$$

Of course, if we take the mechanism $(n>1)$

$$
A_{i}^{\mathrm{I}}+n A_{i}^{\mathrm{II}} \rightarrow(n+1) A_{i}^{\mathrm{I}}, A_{i}^{\mathrm{I}}+A_{i}^{\mathrm{II}} \rightarrow(n+1) A_{i}^{\mathrm{I}},
$$

then we get the equation 


$$
\frac{\partial c}{\partial t}=-k l(n-1) \operatorname{div}\left(c^{n} \nabla c\right)=-k l \frac{n-1}{n+1} \Delta c^{n+1} .
$$

This diffusion process has two properties: first, it goes along gradients and all deviations from the uniform state will increase. Second, this diffusion is slow for small concentrations (the diffusion coefficient goes to 0 when $c$ approaches 0 ) and accelerates with the concentration growth.

The equation $\partial_{t} c=-D \Delta c^{n}(n>1)$ admits a family of self-similar solutions with bounded support, which collapse in finite time. These solutions have the form

$$
c(\tau)=\frac{A}{\rho^{q}} \phi\left(\frac{r}{\rho}\right),
$$

where

- $\tau$ is the time till collapse;

- $q$ is the dimension of space (usually, $q=1,2$ or 3 );

- $\rho$ is the radius of the sphere, outside of which the solution is zero

$$
\rho=B(D \tau)^{\frac{1}{q(n-1)+2}}
$$

- $\phi(\vartheta)=\left(1-\vartheta^{2}\right)^{\frac{1}{n-1}}$ for $\vartheta<1$ and $\phi(\vartheta)=0$ if $\vartheta \geq 1$;

- The constants $A, B$ depend on $q, n$ and the total amount $N=\int c(x) \mathrm{d} x$.

This is the so-called Barenblatt solution [1] for the equation of porous media $\partial_{\tau} c=+D \Delta c^{n}$. Such solutions were used in the analysis of an explosion which starts from a singularity for equations $\partial_{t} c=+D \Delta c^{n}$ (the classical review of self-similar solutions was published by Barenblatt and Zeldovich [2]).

The cell model of diffusion with attraction (1.40) for a finite number of cells of a given size $l$ is a rather regular system of nonlinear ODE, but to first order of the Taylor expansion in $l$ the PDE (1.42) produces a singularity in an arbitrarily short time from smooth initial data. The second order Taylor approximation adds nothing because the even terms in $l$ cancel out if we take into account both the left and right neighbors of the cell. The third order Taylor expansion gives a regularized equation:

$$
\begin{gathered}
J=J=w_{r}-w_{r}^{\prime}=k l c^{2} \frac{\partial}{\partial x}\left(c+\frac{l^{2}}{3} \frac{\partial^{2} c}{\partial x^{2}}\right)+o\left(l^{3}\right) ; \\
\frac{\partial c}{\partial t}=-k l \frac{\partial}{\partial x} c^{2} \frac{\partial}{\partial x}\left(c+\frac{l^{2}}{3} \frac{\partial^{2} c}{\partial x^{2}}\right) .
\end{gathered}
$$

This is an example of the Cahn-Hilliard type equation for spinodal decomposition with the regularizing term $-\operatorname{div}\left(c^{2} \operatorname{grad} \Delta c\right)$. In this equation, the cell size cannot be eliminated by scaling. The length $l$ is the "regularization length". All inhomogeneities of size smaller than $l$ are smoothed by the biharmonic term. 
As we can see, the mass action law and the cell representation of the elementary acts of diffusion give the opportunity to model the Cahn-Hilliard type phase separation. Nevertheless, the approach based on the non-perfect thermodynamic potential (1.25) gives a better representation of the basic physics and does not require complicated elementary processes. Just the simplest Fick scheme,

$$
A_{i}^{\mathrm{I}} \rightarrow A_{i}^{\mathrm{II}}, A_{i}^{\mathrm{II}} \rightarrow A_{i}^{\mathrm{I}}
$$

with the non-perfect Ginzburg-Landau free energy gives the Cahn-Hilliard equation (Sec. 3.2.).

The diffusion mechanism with attraction (1.40) (Fig. 3(c)) differs from the elementary Fick mechanism (Fig. 3(c)) and from the mechanism of jumps to free places (Fig. 3(b)). The dynamical difference is obvious, the diffusion mechanism with attraction generates instabilities of the homogeneous state, clustering and singularities. On the other hand, Fick's law and the mechanism of jumps to free places (Fig. 3(b)) allow a global Lyapunov functional and, in the systems without external fluxes, lead to homogeneous equilibrium.

These mechanisms have also a very important structural difference. If we look at the direct and the space-inverted processes (Fig. 3) then we find that for the first two mechanisms, the spaceinverted processes coincide with the inverse processes, which we get just by inversion of the arrow (or by the exchange $\alpha$ and $\beta$ coefficients in the stoichiometric equations (1.37)). For the elementary processes with attractions (Fig. 3(c)) the inverse processes are processes with repulsion:

$$
3 A_{i}^{\mathrm{II}} \rightarrow A_{i}^{\mathrm{I}}+2 A_{i}^{\mathrm{II}}, 3 A_{i}^{\mathrm{I}} \rightarrow 2 A_{i}^{\mathrm{I}}+A_{i}^{\mathrm{II}} .
$$

The diffusion processes for which space-inverted elementary processes coincide with the inverse processes, have a fundamental property: The entropy production is positive for the corresponding mass action law diffusion equations.

Let us consider a complex diffusion process in a bounded domain with smooth boundary and without external fluxes. We prove the following theorem in Section 2.2.3.

Theorem 2. Let a complex diffusion process consist of elementary processes, which satisfy the following property: the space-inverted elementary process coincides with the inverse process. Then, for the mass action law equation of diffusion (2.25), the principle of detailed balance is valid, the global convex Lyapunov functional exists and the uniform distribution is asymptotically stable.

This global Lyapunov functional may be selected in the form of the (minus) classical entropy, the sum of terms $c \ln c$ for all cells and components, or, for the continuous limit,

$$
\sum_{i} \int c_{i} \ln c_{i} \mathrm{~d} x
$$

A particular case of the dissipation inequality for such processes is inequality (1.22) for the diffusion equations (1.20) that describe diffusion by exchange of positions. It is valid because the exchange mechanism satisfies this fundamental property: the space-inverted elementary processes coincide with the inverse processes. 


\subsubsection{Thermodynamics and Intermediate Complexes}

Thermodynamics is not always a good leader, but it is always a good judge. We cannot create nonlinear equations directly from thermodynamic principles, but we must always check whether our equations satisfy thermodynamics. They should satisfy the thermodynamic restrictions if we do not want to produce a perpetuum mobile in our theory.

We also include some other fundamental restrictions like micro-reversibility in the thermodynamic requirements.

It is not always simple to coordinate the lattice models with thermodynamics, nevertheless it is possible [64, 99].

There are two main approaches for the introduction of thermodynamics into kinetic models. First, we can start from general kinetic equations based on the representation of a complex process as an ensemble of elementary processes with a given simple kinetic law of elementary processes (for example, the mass action law). After that, we will find that the rate constants of the elementary processes are not independent. They must be coordinated to meet the thermodynamic requirements. Therefore, not all the possible kinetic systems are allowed thermodynamically.

Another approach starts from the thermodynamic description of the system. We should find thermodynamic potentials which describe the system under given conditions. We have to know entropy, free energy (Helmholtz energy), or free enthalpy (Gibbs energy) for the proper set of independent variables [14]. After that, we define the rate of elementary process through the thermodynamic functions but with some arbitrariness: some constants remain free of thermodynamic restrictions. These constants are independent for different elementary processes.

Which way is better? It is not a proper question: both are good for their purposes. The first approach (we start from kinetics and then add thermodynamics) is very flexible. In particular, it can be used when thermodynamic restrictions are not needed. For example, when we consider subsystems of open systems like the system of surface components in heterogeneous catalysis, then the constants of elementary processes include additional dependencies on some additional concentrations and are not the "proper" rate constants. Therefore, they do not satisfy the thermodynamic restrictions, and a subsystem may demonstrate non-thermodynamic behavior like non-decaying oscillations or bifurcations.

The second approach is unavoidable for non-perfect systems. The kinetic law of elementary processes depends on the thermodynamic potential. For all perfect systems it is the same mass action law, but any deviation from the perfect thermodynamic function requires its own deviation of the kinetic law from the mass action law [68]. This deviation may be reformulated as the generalized mass action law with activities instead of concentrations but the activities are defined through thermodynamic potentials.

In our work we follow both approaches: First, we formulate the mass action law for diffusion and study this with and without the thermodynamic restrictions. Secondly, we introduce the thermodynamic formalism for diffusion in non-perfect systems. The ideas for both approaches for diffusion were formulated in the early 1980s [53,46]. The detailed analysis of the thermodynamic restrictions on chemical kinetics was performed by Gorban in 1982 [42].

Our work was influenced by the works of N.G. Van Kampen [105], M. Feinberg [29, 30] and 
Horn and Jackson [62]. In 1973, N.G. Van Kampen proposed a general formulation for the rates of irreversible processes as a combination of "unilateral transfer flows". Each unilateral flow transfers energy and particles in one direction. Van Kampen decomposed the total in partial systems, each of which is in equilibrium and therefore possesses a well-defined temperature, entropy, and other thermodynamic quantities. Although the total system $\mathrm{Y}$ is not in equilibrium, it is still possible to attribute an entropy to it. Then Van Kampen studied the unilateral fluxes between subsystems.

We decompose the Van Kampen unilateral processes further and represent them as a collection of essentially one-dimensional elementary processes with the simple kinetic mechanism, the mass action law or the generalized mass action law.

We start from a similar representation of the total system and supplement it with the system of stoichiometric equations of elementary unilateral processes. To find the rate of the elementary processes we use an idea of intermediate complex (compound). This approach is borrowed from the theory of absolute reaction rates but we do not use the special idealization of the reaction pass and postulate the more general microscopic Markov kinetics instead.

If the concentrations of compounds are small and the equilibrium between intermediates and other components is fast (both assumptions are important) then we approach the generalized mass action law, which is very similar to the Marselin-de Donder kinetics and the generalized mass action law studied by Feinberg, Horm and Jackson and other authors [29, 30, 62, 10].

This formalism is very convenient for implementation of the microreversibility consequences in the form of detailed balance conditions [75]. In addition, if there is no microreversibility then the thermodynamic behavior is also guaranteed by the special more general relations between kinetic constants, which follow from the Markov kinetics of intermediate complexes. First, the idea of such relations was proposed by Boltzmann as an answer to the Lorentz objections against Boltzmann's proof of the $H$-theorem. Lorentz stated nonexistence of inverse collisions for polyatomic molecules. Boltzmann did not object to this argument but proposed the "cyclic balance" condition, that means balancing in cycles of transitions between states $S_{1} \rightarrow S_{2} \rightarrow \ldots \rightarrow S_{n} \rightarrow S_{1}$. Almost 100 years later, Cercignani and Lampis [15] demonstrated that the Lorenz arguments are wrong and this Boltzmann new relations are not needed for the polyatomic molecules under the microreversibility conditions. The detailed balance conditions should hold.

Nevertheless, this Boltzmann's idea is very seminal. It was studied further by Heitler [60] and Coester [19] and the results are sometimes cited as the "Heitler-Coestler theorem of semidetailed balance". In 1952 [97] proved these conditions for the Boltzmann equation. For the micro-description he used the $S$-matrix representation, which is in this case equivalent for the Markov microkinetics (see also [112]). Later, this sort of relation was rediscovered for chemical kinetics [30, 62]. The general proof for nonlinear nonequlibrium processes was presented recently [54]. In our analysis of these Boltzmann-...-Stueckelberg relations we follow the later.

We extend the usual stoichiometric equations by additional reactions: an input linear combination of reagents forms a corresponding compound; this compound transforms into another compound that disintegrates into the corresponding output linear combination of reagents:

$$
\sum_{i} \alpha_{\rho i} A_{i} \rightleftharpoons B_{\rho}^{-} \rightarrow B_{\rho}^{+} \rightleftharpoons \sum_{i} \beta_{\rho i} A_{i}
$$

Here $\rho$ is the elementary reaction number. 


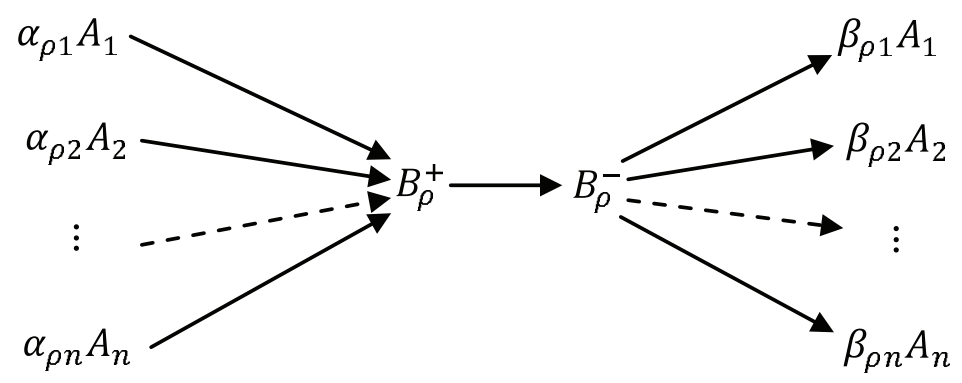

Figure 5: A $2 n$-tail scheme of an extended elementary process (1.44).

It is useful to visualize the reaction scheme. In Fig. 5 we represent the $2 n$-tail scheme of an elementary reaction sequence (1.44). This scheme was proposed in [54].

We assume that the amount of each compound $B_{\rho}$ is small enough to apply the perfect entropy formula, and that the equilibrium between each compound and the corresponding linear combinations of reagents is fast enough to apply the quasiequilibrium approximation [54] (the detailed analysis of this approximation was given in $[52,50,51])$.

The main difference from the Eyring approach [66] is in the model of the hidden reaction of the "activated complexes":

- Eyring used for each reaction one complex with a continuum of energetic states along the "reaction path", whereas we use two compounds (or two states);

- Eyring modeled the reaction of the intermediate complex as a classical motion along the additional coordinate and even added this degree of freedom with classical kinetic energy of this motion to the free energy calculation, whereas we follow the Stueckelberg approach and model this reaction as a first order Markov kinetics, a Markov process with two states.

The difference in the macroscopic consequences of these approaches seems to be not very large because the result of the Eyring approach is one relaxation time approximation for each reaction. From the dynamical point of view, this result coincides with the two-state Markov model.

The main differences may arise in the hints which these approaches give to the microscopic calculation of the macroscopic quantities. In our work, we concentrate on the macroscopic dynamics.

\section{Mass Action Law for Diffusion}

\subsection{Mass Action Law}

\subsubsection{Mass Action Law Kinetic Equations}

This is an auxiliary subsection where we collect main definitions and results about the Mass Action Law (MAL). 
To construct a system of kinetic equations by MAL, one needs the following inputs:

1. A list of components;

2. A list of elementary reactions represented by their stoichiometric equations;

3. A set of reaction rate constants.

The list of components is just a set of symbols (the component names). We usually assume that this set is finite, $A_{1}, A_{2}, \ldots, A_{n}$.

Elementary reactions are given by their stoichiometric equations,

$$
\sum_{i} \alpha_{r i} A_{i} \rightarrow \sum_{i} \beta_{r i} A_{i}
$$

where $r$ is a reaction number, $\alpha_{r i}$ and $\beta_{r i}$ are nonnegative numbers, the stoichiometric coefficients. By default, they are assumed to be integer but, sometimes, there occurs a need in nonnegative real coefficients.

For each elementary reaction (2.1), a stoichiometric vector is defined,

$$
\gamma_{r}: \gamma_{r i}=\beta_{r i}-\alpha_{r i}
$$

This is a "bookkeeping" vector, whose components are "gain minus loss" (or "income minus outcome").

We will also use the loss and gain vectors of elementary reactions: $\alpha_{r}$ (loss) with coordinates $\alpha_{r i}$ and $\beta_{r}$ (gain) with coordinates $\beta_{r i}$. Of course,

$$
\gamma_{r}=\beta_{r}-\alpha_{r}
$$

The stoichiometric matrix $\Gamma$ is the matrix with columns $\gamma_{i}: \Gamma_{i j}=\gamma_{j i}$, the first index in $\Gamma_{i j}$ corresponds to component and the second index corresponds to reaction. Reaction rate constants $k_{r}$ are non-negative numbers. They should be defined for all elementary reactions. For each component $A_{i}$, a real variable, concentration $c_{i}$ is defined. Vector of concentrations $c$ has coordinates $c_{i}$.

The reaction rate for the elementary reaction (2.1) is the following function of $c$

$$
w_{r}=k_{r} \prod_{i=1}^{n} c_{i}^{\alpha_{r i}} .
$$

The MAL kinetic equations are

$$
\frac{\mathrm{d} c}{\mathrm{~d} t}=\sum_{r} \gamma_{r} w_{r}
$$

From the physical point of view, these equations describe isochoric isothermal processes for perfect systems. For non-isochoric or non-isothermal processes it is necessary to introduce the volume (together with pressure) and the enthalpy (together with temperature) explicitly and describe their dynamics. 
For a given reaction mechanism, a linear stoichiometric conservation law is a linear functional $b(c)=\sum_{i} b_{i} c_{i}$ that annihilates all stoichiometric vectors:

$$
b\left(\gamma_{r}\right)=0 \text { for all } r .
$$

The stoichiometric conservation law is strictly positive, if all $b_{i}>0$. The assumption about existence of a positive stoichiometric conservation law plays an important role in the MAL kinetics.

\subsubsection{Existence and Uniqueness of Solutions}

Let in the reaction mechanism all nonzero coordinates of the loss vectors be not less than 1 :

$$
\alpha_{r i} \geq 1 \text { or } \alpha_{r i}=0 .
$$

This assumption is valid, for example, if all the stoichiometric coefficients are nonnegative integers. Let us assume also that there exists a strictly positive stoichiometric conservation law $b$. Then the following existence and uniqueness theorem for the MAL equation holds.

Theorem 1. For any nonnegative initial data $c(0)\left(c_{i}(0) \geq 0\right)$ there exists a unique solution of (2.3) $c(t)$ for all $t>0$. This solution is nonnegative $\left(c_{i}(t) \geq 0\right)$ and satisfies the conservation law: $b(c(t))=b(c(0))$.

This is a well known result (see, for example, [109]). The proof is quite simple. First, of all, let us consider a bounded neighborhood $U$ of the simplex $\Sigma_{0}: c_{i} \geq 0, b(c)=b(c(0))$. The right hand site of the MAL kinetic equations (2.3) has continuous first derivatives and these derivatives are bounded in $U$. Therefore, according to a standard existence and uniqueness theorem its solution exists on some time interval $t \in[0, T]$ and this $T$ is the same for a compact set of initial data $c(0) \in \Sigma_{0} \Subset U$. Secondly, let us mention that if $\gamma_{r i}<0$ then $\alpha_{r i} \geq 1$ and the reaction rate $w_{r}(2.2)$ includes the factor $c_{i}^{\alpha_{r i}}$. Therefore,

$$
\sum_{\gamma_{r i}<0} \gamma_{r i} w_{r}=c_{i} g(c)
$$

where $g(c)$ is continuous function.

If $c_{i} \rightarrow 0$ then $\sum_{\gamma_{r i}<0} \gamma_{r i} w_{r} \rightarrow 0$. If $c_{i}=0$ then $\dot{c}_{i}=\sum_{r} \gamma_{r i} w_{r} \geq 0$. Therefore, the simplex $\Sigma_{0}$ is positively invariant with respect to equations (2.3): the existent solutions $c(t)$ do not leave this simplex for $t>0$ if $c(0) \in \Sigma_{0}$. Finally, this implies global existence of solutions in $\Sigma_{0}$.

Both conditions of existence of a strictly positive stoichiometric conservation law $b$ and of coordinates of the loss vectors, $\alpha_{i}=0$ or $\alpha_{i} \geq 1$ are significant. Just for example, we can consider mechanisms that violate these conditions: $2 A \rightarrow 3 A$ and $\frac{1}{2} A \rightarrow A$. For the first mechanism, $\dot{c}=k c^{2}$ and there is no global existence, for the second system, $\dot{c}=k \sqrt{c}$ and there is no uniqueness of solution. 


\subsubsection{Detailed Balance}

The expected behavior of a system of physical or chemical kinetics is simple in the absence of external fluxes: everything goes to equilibrium. A Lyapunov function for this relaxation is the corresponding thermodynamic potential.

The MAL kinetics (2.3) do not assume any thermodynamic properties "from scratch". Moreover, this class of kinetic equations is so rich that it is dense in the class of all smooth semidynamical systems in $\Sigma_{0}$ for a given conservation law $b$ [46]. Additional assumptions are needed to guarantee the thermodynamic behavior.

The most celebrated sufficient condition for the thermodynamic behavior of the MAL kinetics is the principle of detailed balance. This principle, as a realization of microreversibility was known for the Boltzmann equation [6] (since his proof of the $H$-theorem in 1872) long before Onsager's reciprocal relations $[83,84]$. A. Einstein used this principle for the linear kinetics of emission and absorption of radiation [25]. In 1901, R. Wegscheider published analysis of detailed balance for chemical kinetics [113].

To formulate the principle of detailed balance, it is convenient to join pairs of direct and inverse elementary reactions in (2.1) and write

$$
\sum_{i} \alpha_{r i} A_{i} \rightleftharpoons \sum_{i} \beta_{r i} A_{i}
$$

If the inverse reaction does not exist in the original mechanism, we formally add it but assume that its rate constant is zero. We mark quantities for the direct and inverse reactions by the upper indexes $^{+}$and $^{-}$and write the MAL reaction rate:

$$
\begin{aligned}
& w_{r}=w_{r}^{+}-w_{r}^{-}, \\
& w_{r}^{+}=k_{r}^{+} \prod_{i=1}^{n} c_{i}^{\alpha_{r i}}, w_{r}^{-}=k_{r}^{-} \prod_{i=1}^{n} c_{i}^{\beta_{r i}} .
\end{aligned}
$$

For the MAL kinetics the principle of detailed balance is: there exists a strictly positive point of detailed balance that is such vector of concentration $c^{*}$ that $c_{i}^{*}>0$ and

$$
w_{r}^{+}\left(c^{*}\right)=w_{r}^{-}\left(c^{*}\right)\left(=w_{r}^{*}>0\right) .
$$

This means that at least at one positive point the direct elementary processes are equilibrated by the inverse elementary processes.

Existence of one such point implies that all equilibria are also points of detailed balance (2.6) and, moreover, there exists a global Lyapunov function that has the form of relative entropy. This is, precisely, the analogue of the Boltzmann $H$-theorem for the MAL kinetics.

For the formulation, use and proof of this theorem, it is convenient to rewrite the formulas for the direct and inverse reaction rates (2.5) using $c^{*}, w_{r}^{*}$ and the detailed balance relations (2.6):

$$
\begin{aligned}
& w_{r}=w_{r}^{+}-w_{r}^{-}, \\
& w_{r}^{+}=w_{r}^{*} \prod_{i=1}^{n}\left(\frac{c_{i}}{c_{i}^{*}}\right)^{\alpha_{r i}}, w_{r}^{-}=w_{r}^{*} \prod_{i=1}^{n}\left(\frac{c_{i}}{c_{i}^{*}}\right)^{\beta_{r i}} .
\end{aligned}
$$


The Lyapunov function is:

$$
G=\sum_{i} c_{i}\left(\ln \left(\frac{c_{i}}{c_{i}^{*}}\right)-1\right)+\sum_{i} c_{i}^{*}
$$

Here, the last constant term is added to satisfy $G\left(c^{*}\right)=0$.

The partial derivatives of $G$ (the analogs of chemical potentials) are

$$
\frac{\partial G}{\partial c_{i}}=\ln \left(\frac{c_{i}}{c_{i}^{*}}\right)
$$

Therefore, we have one more form for the MAL kinetic law with detailed balance (2.7):

$$
\begin{aligned}
& w_{r}=w_{r}^{+}-w_{r}^{-} \\
& w_{r}^{+}=w_{r}^{*} \exp \left(\sum_{i} \alpha_{r i} \frac{\partial G}{\partial c_{i}}\right)=w_{r}^{*} \exp \left(\alpha_{r}, \nabla_{c} G\right), \\
& w_{r}^{-}=w_{r}^{*} \exp \left(\sum_{i} \beta_{r i} \frac{\partial G}{\partial c_{i}}\right)=w_{r}^{*} \exp \left(\beta_{r}, \nabla_{c} G\right),
\end{aligned}
$$

It is worth mentioning that

$$
\frac{w_{r}^{+}}{w_{r}^{-}}=\exp \left[-\left(\gamma_{r}, \nabla_{c} G\right)\right] .
$$

For the time derivative of $G$ due to the MAL kinetics (2.3) with the detailed balance, simple algebra gives the dissipation inequality:

$$
\frac{\mathrm{d} G}{\mathrm{~d} t}=\sum_{r} w_{r}\left(\gamma_{r}, \nabla_{c} G\right)=-\sum_{r}\left(w_{r}^{+}-w_{r}^{-}\right) \ln \left(\frac{w_{r}^{+}}{w_{r}^{-}}\right) \leq 0 .
$$

The last inequality holds because $\ln x$ is a strictly monotone function and $\ln x-\ln y$ has the same sign as $x-y$ has. Obviously, $\left.\dot{G}\right|_{c}=0$ if and only if $c$ is a point of detailed balance. This equilibrium point may be different from the point $c^{*}$, which was used for the definition. All the positive points of detailed balance for the MAL system (2.7) form a smooth manifold with dimension

$$
n-\operatorname{rank}\left\{\gamma_{1}, \gamma_{2}, \ldots\right\},
$$

where $n$ is the number of components and $\operatorname{rank}\left\{\gamma_{1}, \gamma_{2}, \ldots\right\}$ is the rank of the system of the stoichiometric vectors for the given reaction mechanism.

If we fix values of all stoichiometric linear conservation laws then the strictly positive point of detailed balance is unique for the given values. Indeed, the dissipation inequality is valid for every pair of mutually inverse reactions and all the terms $w_{r}\left(\gamma_{r}, \nabla_{c} G\right)$ in $\dot{G}(2.11)$ are non-positive. Therefore, at any strictly positive equilibrium point $c^{\mathrm{eq}},\left(\gamma_{r}, \nabla_{c} G\right)=0$ for all $r$. This means that 
$c^{\text {eq }}$ is a critical point of $G$ in $c^{\mathrm{eq}}+\operatorname{span}\left\{\gamma_{1}, \gamma_{2}, \ldots\right\}$. The function $G$ is strictly convex at any positive point: its Hessian is positive definite,

$$
\frac{\partial^{2} G}{\partial c_{i} \partial c_{j}}=\frac{1}{c_{i}} \delta_{i j},
$$

where $\delta_{i j}$ is the Kronecker delta. Therefore, there may exist only one positive critical point of $G$ on a linear manifold.

Due to the logarithmic singularity of the gradients at the boundary of $\mathbb{R}_{+}$(where some of $\left.c_{i}=0\right), G$ achieves its global minimum in

$$
\left(c^{\mathrm{eq}}+\operatorname{span}\left\{\gamma_{1}, \gamma_{2}, \ldots\right\}\right) \bigcap \mathbb{R}_{+}^{n}
$$

at a positive point. This point is a positive point of detailed balance.

For any positive vector, $c^{0}$, the polyhedron

$$
\mathcal{V}=\left(c^{0}+\operatorname{span}\left\{\gamma_{1}, \gamma_{2}, \ldots\right\}\right) \bigcap \mathbb{R}_{+}^{n},
$$

is positively invariant with respect to (2.3). For systems with detailed balance it includes one and only one positive point of detailed balance. This was first demonstrated by Zeldovich in 1938 (reprinted in 1996 [118]). In our analysis we mainly follow [109].

\subsubsection{Complex Balance}

In this subsection we consider the direct and inverse reactions separately as we did it before in (2.1), (2.2). Detailed balance is s sufficient but not necessary condition of the thermodynamic behavior. The simple example of thermodynamic behavior gives any monomolecular (linear) reaction mechanism, which consists of reactions $A_{i} \rightarrow A_{j}$. Let us use notation $k_{j i}$ for this reaction rate constant.

The MAL equations for a monomolecular reaction mechanism are

$$
\frac{\mathrm{d} c_{i}}{\mathrm{~d} t}=\sum_{j, j \neq i}\left(k_{i j} c_{j}-k_{j i} c_{i}\right) .
$$

Let $c^{0}$ be a strictly positive steady state for these equations (not necessarily a point of detailed balance):

$$
\sum_{j, j \neq i} k_{i j} c_{j}^{0}=c_{i}^{0} \sum_{j, j \neq i} k_{j i}
$$

With this $c^{0}$ we can rewrite the second term in (2.12):

$$
\sum_{j, j \neq i} k_{j i}=\sum_{j, j \neq i} k_{i j} \frac{c_{j}^{0}}{c_{i}^{0}}
$$




$$
\sum_{j, j \neq i} k_{j i} c_{i}=\sum_{j, j \neq i} k_{i j} c_{j}^{0} \frac{c_{i}}{c_{i}^{0}} .
$$

Therefore, the kinetic equations (2.12) have the equivalent form for given $c^{0}$ :

$$
\frac{\mathrm{d} c_{i}}{\mathrm{~d} t}=\sum_{j, j \neq i} k_{i j} c_{j}^{0}\left(\frac{c_{j}}{c_{j}^{0}}-\frac{c_{i}}{c_{i}^{0}}\right) .
$$

Then we can define

$$
G=\sum_{i} c_{i}\left(\ln \left(\frac{c_{i}}{c_{i}^{0}}\right)-1\right) .
$$

After simple transformations, we find that due to (2.13)

$$
\begin{gathered}
\frac{\mathrm{d} G}{\mathrm{~d} t}=\sum_{i j i \neq j} k_{i j} c_{j}^{0}\left[\frac{c_{i}}{c_{i}^{0}}\left(\ln \left(\frac{c_{i}}{c_{i}^{0}}\right)-1\right)-\frac{c_{j}}{c_{j}^{0}}\left(\ln \left(\frac{c_{j}}{c_{j}^{0}}\right)-1\right)\right. \\
\left.+\ln \left(\frac{c_{i}}{c_{i}^{0}}\right)\left(\frac{c_{j}}{c_{j}^{0}}-\frac{c_{i}}{c_{i}^{0}}\right)\right] \leq 0 .
\end{gathered}
$$

To prove this formula, it is worth mentioning that for any $n$ numbers $f_{i}$,

$$
\sum_{i j i \neq j} k_{i j} c_{j}^{0}\left(f_{i}-f_{j}\right)=0 .
$$

This gives us the first two terms in the square brackets with

$$
f_{i}=\frac{c_{i}}{c_{i}^{0}}\left(\ln \left(\frac{c_{i}}{c_{i}^{0}}\right)-1\right) .
$$

The last term,

$$
\ln \left(\frac{c_{i}}{c_{i}^{0}}\right)\left(\frac{c_{j}}{c_{j}^{0}}-\frac{c_{i}}{c_{i}^{0}}\right)
$$

appears in the straightforward computation of the time derivative of $G$ due to kinetic equations (2.13).

The expressions in square brackets in (2.15) have the form

$$
f(a)-f(b)+f^{\prime}(a)(b-a)
$$

for the convex function $f(x)=x(\ln x-1)$. This expression is always non-positive because of Jensen's inequality.

Linear MAL kinetics can obviously violate the principle of detailed balance. For example, an irreversible cycle

$$
A_{1} \rightarrow A_{2} \rightarrow \ldots \rightarrow A_{n} \rightarrow A_{1}
$$

has always a positive steady state but never has a positive point of detailed balance if $n>2$. 
There is a nice generalization of the dissipation inequality (2.15) for the nonlinear MAL equations under some algebraic conditions on the kinetic constants. These conditions are strictly weaker than the principle of detailed balance. They were discovered for the Boltzmann equation by Stueckelberg [97] in 1952 and called later the "complex balancing condition" for the general MAL $[62,30]$.

To formulate this condition for the MAL kinetics, let us start from the function $G$ (2.14) and look for conditions that guarantee the inequality $\dot{G} \leq 0$.

For a given $c^{0}$, we can rewrite the MAL reaction rate in the form

$$
w_{r}=\varphi_{r} \exp \left(\alpha_{r}, \nabla_{c} G\right)=\varphi_{r} \prod_{i}\left(\frac{c_{i}}{c_{i}^{0}}\right)^{\alpha_{r i}},
$$

where $\varphi_{r}=k_{r} \prod_{i}\left(c_{i}^{0}\right)^{\alpha_{r i}}=w_{r}\left(c^{0}\right)$.

It is convenient to express $\dot{G}$ using an auxiliary function $\theta$ of an auxiliary variable $\lambda$ : for any concentration vector,

$$
\theta(\lambda)=\sum_{r} \varphi_{r} \exp \left[\lambda\left(\alpha_{r}, \nabla_{c} G\right)+(1-\lambda)\left(\beta_{r}, \nabla_{c} G\right)\right] .
$$

This function is convenient because

$$
\frac{\mathrm{d} \theta(\lambda)}{\mathrm{d} \lambda}=-\sum_{r} \varphi_{r}\left(\mu,\left(\beta_{r}-\alpha_{r}\right)\right) \exp \left[\lambda\left(\alpha_{r}, \nabla_{c} G\right)+(1-\lambda)\left(\beta_{r}, \nabla_{c} G\right)\right] .
$$

In this notation,

$$
\dot{G}=-\theta^{\prime}(1) .
$$

The function $\theta(\lambda)$ is a sum of exponents. It is convex $\left(\theta^{\prime \prime}(\lambda) \geq 0\right)$. Therefore, if $\theta(0)=\theta(1)$ then $\theta^{\prime}(1) \geq 0$.

This condition, $\theta(0)=\theta(1)$ (for all positive $c$ ) is called the complex balancing condition and it is sufficient for the dissipation inequality:

$$
\dot{G}=-\theta^{\prime}(1) \leq 0 \text {. }
$$

The principle of detailed balance for the MAL equations has a form of existence of a special equilibrium point, a point of detailed balance. This existence implies important dynamical properties in the whole of $\mathbb{R}_{+}^{n}$ because of the very "rigid" monomial structure of MAL.

The complex balancing condition also can be formulated as existence of a special "point of complex balance". Let us reformulate it in this way.

Some vectors $\alpha_{r}, \beta_{r}$ for a given reaction mechanism may coincide. Let us denote by $\left\{y_{1}, \ldots, y_{q}\right\}$ the set of all different vectors $\alpha_{r}, \beta_{r}$. For each $y_{i}$ we define $R_{i}^{+}=\left\{r \mid \alpha_{r}=y_{i}\right\}, R_{i}^{-}=\left\{r \mid \beta_{r}=\right.$ $\left.y_{i}\right\}$. In this notation, 


$$
\begin{aligned}
& \theta(1)=\sum_{i}\left(\sum_{r \in R_{i}^{+}} w_{r}\left(c^{0}\right)\right) \exp \left(y_{i}, \nabla_{c} G\right), \\
& \theta(0)=\sum_{i}\left(\sum_{r \in R_{i}^{-}} w_{r}\left(c^{0}\right)\right) \exp \left(y_{i}, \nabla_{c} G\right) .
\end{aligned}
$$

For any finite set of (different) vectors $\left\{y_{1}, \ldots, y_{q}\right\}$ the correspondent functions $\exp \left(y_{i}, \nabla_{c} G\right)$ of $c \in \mathbb{R}_{+}^{n}$ are linearly independent because the Hessian of $G$ is strictly positive definite. Therefore, the condition $\theta(0)=\theta(1)$ (for all positive $c$ ) is equivalent to

$$
\sum_{r \in R_{i}^{+}} w_{r}\left(c^{0}\right)=\sum_{r \in R_{i}^{-}} w_{r}\left(c^{0}\right) .
$$

The point $c^{0}$ that satisfies (2.20) is called the point of complex balance and the complex balancing condition means that there exists such a strictly positive point of complex balance. In this case, the dissipation inequality, $\dot{G} \leq 0$, with $G$ defined by (2.14) holds for all positive points.

Of course, a point of detailed balance is a point of complex balance as well. The reverse statement is not valid: for example, all the positive steady states of linear MAL kinetics (2.12) are the points of complex balance but they are not necessarily the points of detailed balance.

The microscopic background for detailed balance is microreversibility, i.e. invariance of the microscopic classical or quantum equations with respect to the time inversion.

The microscopic backgrounds for complex balance were formulated by Stueckelberg as unitarity of the $S$-matrix [97]. It is necessary to add that the validity of the scattering (or Markov) model of elementary reactions is also needed: see [54] and discussion in Section 3.

\subsection{Mass Action Cell-Jump Formalism}

\subsubsection{Stoichiometry of Diffusion Jumps}

We represent the physical space as a network of compartments. Each compartment is modeled as a cubic cell with an edge size $l$. The stoichiometric equations of diffusion describe interaction of two neighboring cells. To distinguish the quantities related to these two cells we use the upper indexes I and II (Fig. 2).

The general stoichiometric equation for an elementary event of diffusion is (1.37)

$$
\sum_{i} \alpha_{r i}^{\mathrm{I}} A_{i}^{\mathrm{I}}+\sum_{i} \alpha_{r i}^{\mathrm{II}} A_{i}^{\mathrm{II}} \rightarrow \sum_{i} \beta_{r i}^{\mathrm{I}} A_{i}^{\mathrm{I}}+\sum_{i} \beta_{r i}^{\mathrm{II}} A_{i}^{\mathrm{II}} .
$$

Coefficients $\alpha_{r i}^{\mathrm{I}, \mathrm{II}}, \beta_{r i}^{\mathrm{I}, \mathrm{II}}$ are nonnegative. Usually, we assume that they are integers but in some situations real numbers are needed. 
Elementary events (2.21) describe diffusion and do not include the transformation of components (reactions). Therefore, the total amounts of each component $A_{i}$ coincide in the left and the right hand sides of (2.21):

$$
\alpha_{r i}^{\mathrm{I}}+\alpha_{r i}^{\mathrm{I}}=\beta_{r i}^{\mathrm{I}}+\beta_{r i}^{\mathrm{II}} .
$$

Each elementary process (2.21) has two loss vectors, $\alpha_{r}^{\mathrm{I}, \mathrm{II}}$ with coordinates $\alpha_{r i}^{\mathrm{I}, \mathrm{II}}$ and two output vectors, $\beta_{r}^{\mathrm{I}, \mathrm{II}}$ with coordinates $\beta_{r i}^{\mathrm{I}, \mathrm{II}}$. Because of the conservation of particles of all types (2.22), the stoichiometric vectors of processes for the cells differ just by the sign of coordinates:

$$
\gamma_{r}=\gamma_{r}^{\mathrm{I}}=\gamma_{r}^{\mathrm{II}}=\beta_{r}^{\mathrm{I}}-\alpha_{r}^{\mathrm{I}} .
$$

We define here a mechanism of diffusion as a system of stoichiometric equations for elementary events. The simple and basic examples are:

- Fick's diffusion, $A_{i}^{\mathrm{I}} \rightarrow A_{i}^{\mathrm{II}}, A_{i}^{\mathrm{II}} \rightarrow A_{i}^{\mathrm{I}}$;

- The exchange of particles, $A_{i}^{\mathrm{I}}+A_{j}^{\mathrm{II}} \rightarrow A_{i}^{\mathrm{II}}+A_{j}^{\mathrm{I}}$;

- Clustering (diffusion with attraction), $A_{i}^{\mathrm{I}}+s A_{i}^{\mathrm{II}} \rightarrow(s+1) A_{i}^{\mathrm{II}}, s A_{i}^{\mathrm{I}}+A_{i}^{\mathrm{II}} \rightarrow(s+1) A_{i}^{\mathrm{I}}$, $s>1$

- Diffusion with repulsion, $(s+1) A_{i}^{\mathrm{I}} \rightarrow s A_{i}^{\mathrm{I}}+A_{i}^{\mathrm{II}},(s+1) A_{i}^{\mathrm{I}} \rightarrow A_{i}^{\mathrm{I}}+s A_{i}^{\mathrm{II}},(s>0)$.

Formally, diffusion with repulsion is the time-inverted process of diffusion with attraction (the porous medium model) but for $s=1$ diffusion with attraction has no sense (the exactly uniform state cannot produce the nonuniform distribution). Therefore, the restrictions on $s$ are different.

\subsubsection{MAL Equations for Diffusion}

Let us consider the system of stoichiometric equations (2.21) as a reaction mechanism for MAL (2.1). If we apply MAL then the rate of the elementary diffusion process is

$$
w_{r}\left(c^{\mathrm{I}}, c^{\mathrm{II}}\right)=k_{r} \prod_{i}\left(c_{i}^{\mathrm{I}}\right)^{\alpha_{r i}^{\mathrm{I}}} \prod_{i}\left(c_{i}^{\mathrm{II}}\right)^{\alpha_{r i}^{\mathrm{II}}} .
$$

For example, for Fick's diffusion, we have two elementary processes, $A_{i}^{\mathrm{I}} \rightarrow A_{i}^{\mathrm{II}}$ and $A_{i}^{\mathrm{II}} \rightarrow A_{i}^{\mathrm{I}}$. The corresponding reaction rates are $k_{1} c_{i}^{\mathrm{I}}$ and $k_{2} c_{i}^{\mathrm{II}}$.

The composition of each cell is vector $N^{\mathrm{I}, \mathrm{II}}$. Components of this vector, $N_{i}^{\mathrm{I}, \mathrm{II}}=V^{\mathrm{I}, \mathrm{II}} c_{i}^{\mathrm{I}, \mathrm{II}}$ are amounts of $A_{i}$ in the corresponding cell and $V^{\mathrm{I} \text {,II }}$ are volumes of cells. We describe the dynamics of the compositions of two cells by the equations:

$$
\frac{d N^{\mathrm{I}}}{d t}=-\frac{d N^{\mathrm{II}}}{d t}=S^{\mathrm{I}, \mathrm{II}} \sum_{r} \gamma_{r} w_{r}\left(c^{\mathrm{I}}, c^{\mathrm{II}}\right),
$$

where $S^{\mathrm{I}, \mathrm{II}}$ is the area of the boundary between cells I and II. If there are many cells then

$$
\frac{d N^{\mathrm{I}}}{d t}=\sum_{\mathrm{J}} S^{\mathrm{I}, \mathrm{J}} \sum_{r} \gamma_{r} w_{r}\left(c^{\mathrm{I}}, c^{\mathrm{J}}\right),
$$


with summation through all interacting pairs $(\mathrm{I}, \mathrm{J})$.

For example, for Fick's diffusion, we have two elementary processes, $A_{i}^{\mathrm{I}} \rightarrow A_{i}^{\mathrm{II}}$ and $A_{i}^{\mathrm{II}} \rightarrow A_{i}^{\mathrm{I}}$. The corresponding reaction rates are $k_{1} c_{i}^{\mathrm{I}}$ and $k_{2} c_{i}^{\mathrm{II}}$. Equations (2.24) give

$$
\frac{d N_{i}^{\mathrm{I}}}{d t}=-S^{\mathrm{I}, \mathrm{II}} k_{1} c_{i}^{\mathrm{I}}+S^{\mathrm{I}, \mathrm{II}} k_{2} c_{i}^{\mathrm{II}} .
$$

For several pairs, let us mention the symmetry in pairs: $k_{1}=k_{2}=k$ (we discuss this symmetry in more detail in the next subsection):

$$
\frac{d N_{i}^{\mathrm{I}}}{d t}=\sum_{J} k S^{\mathrm{I}, \mathrm{J}}\left(c_{i}^{\mathrm{J}}-c_{i}^{\mathrm{I}}\right) .
$$

For example, on a straight line (two neighbors), this equation gives

$$
\frac{d N_{i}^{\mathrm{I}}}{d t}=k S^{\mathrm{I}, \mathrm{J}}\left(c_{i}^{\mathrm{I}+1}+c_{i}^{\mathrm{I}-1}-2 c_{i}^{\mathrm{I}}\right) .
$$

From this expression, the proper scaling of $k$ with the cell size is obvious:

$$
\frac{d c_{i}^{\mathrm{I}}}{d t}=k \frac{S^{\mathrm{I}, \mathrm{J}} l^{2}}{V} \frac{c_{i}^{\mathrm{I}+1}+c_{i}^{\mathrm{I}-1}-2 c_{i}^{\mathrm{I}}}{l^{2}}
$$

the fraction $c_{i}^{\mathrm{I}+1}+c_{i}^{\mathrm{I}-1}-2 c_{i}^{\mathrm{I}} / l^{2}$ approximates the second derivative and, hence, $k S^{\mathrm{I}, \mathrm{J}} l^{2} / V=$ const.

For a cubic cell, $V=S l$ and $k l=$ const.

\subsubsection{Space Symmetry and Time Symmetry}

The system of elementary events should be symmetric with respect to space-inversion. For each elementary process (2.21) a space-inverted process is defined just by changing I to II and vice versa. We mark the quantities for the space-inverted processes by '. For example,

$$
\gamma^{\prime}=-\gamma
$$

Space inversion is an involution: if we apply it two times then we return to the original process.

The key condition is: the rate functions for the space-inverted processes should differ just by transposition by vectors of variables, $c^{\mathrm{I}}, c^{\mathrm{II}}(1.39)$ :

$$
w_{r}^{\prime}\left(c^{\mathrm{I}}, c^{\mathrm{II}}\right)=w_{r}\left(c^{\mathrm{II}}, c^{\mathrm{I}}\right) .
$$

For MAL this means that $k_{r}=k_{r}^{\prime}$.

The requirement of space symmetry distinguishes diffusion from various types of advection and transport driven by external force. This condition is necessary for existence of diffusion equations when the cell size $l \rightarrow 0$ (see the next subsection). 
Inversion in time differs, in general, from the inversion in space. For example, for the elementary process $3 A^{\mathrm{I}} \rightarrow 2 A^{\mathrm{I}}+A^{\mathrm{II}}$ the space inversion gives $2 A^{\mathrm{II}} \rightarrow A^{\mathrm{I}}+A^{\mathrm{II}}$ (we exchange the upper indexes, $\mathrm{I} \rightarrow \mathrm{II}$ and $\mathrm{II} \rightarrow \mathrm{I}$ ), and the inversion of elementary events ( $T$-transformation) gives $2 A^{\mathrm{I}}+A^{\mathrm{II}} \rightarrow 3 A^{\mathrm{I}}$ (here, we change direction of arrow). We have to stress that inversion in time assumes micro-reversion. At the macroscopic level it does not mean change $t$ to $-t$ in the kinetic equations but the transformation of the direct processes into reverse ones (inversion of collisions, for example).

Time symmetry (microreversibility) means that the principle of detailed balance is valid. In this case, all the consequences of the principle of detailed balance are applicable (Section 2.1.3.), a global Lyapunov functional exists, and every positive equilibrium is a point of detailed balance.

Microreversibility and symmetry of space are independent properties of the system. Nevertheless, for some elementary processes, the space-inverted process coincides with the reverse (the time-inverse) process. If a diffusion mechanism is constructed from such processes then the symmetry in space is equivalent to symmetry in time (to the principle of detailed balance).

This specific class of diffusion mechanisms includes such mechanisms as Fick's diffusion or the diffusion by exchange of particle positions.

Indeed, for Fick's diffusion, when we exchange the upper indexes in an elementary process $A^{\mathrm{I}} \rightarrow A^{\mathrm{II}}$ (inversion in space) then we get the reverse process as well, $A^{\mathrm{II}} \rightarrow A^{\mathrm{I}}$ (inversion of arrows). Analogously, for $A^{\mathrm{I}}+B^{\mathrm{II}} \rightarrow A^{\mathrm{II}}+B^{\mathrm{I}}$ inversion of space gives the reverse process as well: $A^{\mathrm{II}}+B^{\mathrm{I}} \rightarrow A^{\mathrm{I}}+B^{\mathrm{II}}$.

This fundamental property is formulated in the following theorem.

Theorem 2. Let a complex diffusion process consist of elementary processes, which satisfy the following property: the space-inverted elementary process coincides with the inverse process. Then, for the mass action law equation of diffusion (2.25), the principle of detailed balance is valid, the global convex Lyapunov functional exists and the uniform distribution is asymptotically stable.

Indeed, due to space symmetry, a uniform distribution is an equilibrium and each process is equilibrated at this state by its space-inverted process. At the same time, this distribution is a point of detailed balance. Therefore, the results about the principle of detailed balance are applicable.

\subsubsection{Arrested Diffusion and Boundary Equilibria}

Existence of a uniform distribution, which is a point of detailed balance, existence of the global Lyapunov function and asymptotic stability of the uniform equilibrium distributions do not mean that there exist no nonuniform equilibria.

The phenomenon of boundary equilibria is well known. For example, an autocatalytic reversible reaction $A+B \rightleftharpoons 2 A$ has two equilibria for a given value of a stoichiometric conservation law, $b=c_{A}+c_{B}=$ const. One equilibrium is strictly positive, with positive concentrations of $A$ and $B$. Another is a boundary equilibrium, $c_{A}=0, c_{B}=b$. The positive equilibrium is asymptotically stable, the boundary equilibrium is unstable but if the initial state is near the boundary $\left(c_{A}\right.$ is close to zero) then slow relaxation occurs [43], and the motion may be arrested for a long time near this state. 
There are well known effects of arrested diffusion caused by changing of temperature. The solid solutions show the effects of diffusion, which has been arrested by chilling below a threshold temperature in a very short time [74].

Kinetic effects of arrested diffusion are also possible. For example, let us consider the diffusion mechanism by jumps to free places (Fig. 3(b)): any distribution of components $A_{i}$ for zero concentrations of free places $Z$ is stationary, and for a small concentration of free places diffusion is slow.

For effects of arrested diffusion, the average concentration of free places should not be small. There may be, for example, a layer of particles with low mobility between a dense island of particles with high mobility and an island of free places. Formally, it is possible to construct many such situations. All of them may be characterized as follows: either a small change of concentrations or a small change of constants (or both) lead the system to a nonuniform equilibrium. At this nonuniform equilibrium some of the concentrations in some cells take zero values and, therefore, some of fluxes are also zero. Because of the appearance of these zero concentrations, such an equilibrium is called a boundary equilibrium.

\subsection{Continuous Diffusion Equation}

\subsubsection{MAL Diffusion Flux}

Let us consider an elementary process together with its space-inverted process

$$
\begin{aligned}
& \sum_{i} \alpha_{r i}^{\mathrm{I}} A_{i}^{\mathrm{I}}+\sum_{i} \alpha_{r i}^{\mathrm{II}} A_{i}^{\mathrm{II}} \rightarrow \sum_{i} \beta_{r i}^{\mathrm{I}} A_{i}^{\mathrm{I}}+\sum_{i} \beta_{r i}^{\mathrm{II}} A_{i}^{\mathrm{II}}, \\
& \sum_{i} \alpha_{r i}^{\mathrm{I}} A_{i}^{\mathrm{II}}+\sum_{i} \alpha_{r i}^{\mathrm{II}} A_{i}^{\mathrm{I}} \rightarrow \sum_{i} \beta_{r i}^{\mathrm{I}} A_{i}^{\mathrm{II}}+\sum_{i} \beta_{r i}^{\mathrm{II}} A_{i}^{\mathrm{I}} .
\end{aligned}
$$

The reaction rates are

$$
\begin{aligned}
& w_{r}\left(c^{\mathrm{I}}, c^{\mathrm{II}}\right)=k_{r} \prod_{i}\left(c_{i}^{\mathrm{I}}\right)^{\alpha_{r i}^{\mathrm{I}}} \prod_{i}\left(c_{i}^{\mathrm{II}}\right)^{\alpha_{r i}^{\mathrm{II}}}, \\
& w_{r}^{\prime}\left(c^{\mathrm{I}}, c^{\mathrm{II}}\right)=w_{r}\left(c^{\mathrm{II}}, c^{\mathrm{I}}\right)=k_{r} \prod_{i}\left(c_{i}^{\mathrm{II}}\right)^{\alpha_{r i}^{\mathrm{I}}} \prod_{i}\left(c_{i}^{\mathrm{I}}\right)^{\alpha_{r i}^{\mathrm{II}}},
\end{aligned}
$$

where we take $k_{r}^{\prime}=k_{r}$ due to the symmetry in space.

To first order in $l$, the flux vector for $A_{i}$ in this process is

$$
\begin{aligned}
J_{r i} & =-\gamma_{r i}\left[w_{r}(c(x), c(x+l))-w_{r}(c(x+l), c(x))\right] \\
& =-l \gamma_{r i} \sum_{j}\left(\left.\frac{\partial w_{r}\left(c^{\mathrm{I}}, c^{\mathrm{II}}\right)}{\partial c_{j}^{\mathrm{II}}}\right|_{c^{\mathrm{I}}=c^{\mathrm{II}}=c(x)}-\left.\frac{\partial w_{r}\left(c^{\mathrm{I}}, c^{\mathrm{II}}\right)}{\partial c_{j}^{\mathrm{I}}}\right|_{c^{\mathrm{I}}=c^{\mathrm{II}}=c(x)}\right) \nabla c_{j}(x) \\
& =-l \gamma_{r i} w_{r}(c(x), c(x)) \sum_{j} \frac{\alpha_{r j}^{\mathrm{II}}-\alpha_{r j}^{\mathrm{I}}}{c_{j}} \nabla c_{j}(x) \\
& =-l k \gamma_{r i}\left(\prod_{q} c_{q}^{\alpha_{r q}^{\mathrm{I}}+\alpha_{r q}^{\mathrm{II}}}\right) \sum_{j} \frac{\alpha_{r j}^{\mathrm{II}}-\alpha_{r j}^{\mathrm{I}}}{c_{j}} \nabla c_{j}(x) .
\end{aligned}
$$


Here, $\gamma_{r i}=\beta_{r i}^{\mathrm{I}}-\alpha_{r i}^{\mathrm{I}}$ (input minus output in the first cell); the minus in front of the formula appears because the direction of flux from cell I to cell II (from $x$ to $(x+l)$ ) is positive.

The factor $1 / c_{j}$ never leads to a singularity in the flux because $c_{j}$ enters in the monomial $\prod_{q} c_{q}^{\alpha_{r q}^{\mathrm{I}}+\alpha_{r q}^{\mathrm{II}}}$ with the power $\alpha_{r j}^{\mathrm{I}}+\alpha_{r j}^{\mathrm{II}}$. This power is strictly positive if the coefficient $\left(\alpha_{r j}^{\mathrm{II}}-\alpha_{r j}^{\mathrm{I}}\right)$ is not zero.

The proper scaling of $k$ for grid refinement or coarsening is $k l=d=$ const in order not to change the first order expression for flux (2.29).

According to (2.29), the matrix of diffusion coefficients for the elementary process (2.27) (together with its space-inverted process) is

$$
D_{r i j}(c)=d\left(\prod_{q} c_{q}^{\alpha_{r q}^{\mathrm{I}}+\alpha_{r q}^{\mathrm{II}}}\right) \frac{\gamma_{r i}\left(\alpha_{r j}^{\mathrm{II}}-\alpha_{r j}^{\mathrm{I}}\right)}{c_{j}},
$$

where $d=\operatorname{const}(=k l)$.

The corresponding diffusion equations have the divergent form:

$$
\frac{\partial c}{\partial t}=\operatorname{div}(D(c) \nabla c)
$$

where $c$ is the vector of concentrations and $D$ is the matrix of diffusion coefficients (2.30).

It might be useful to represent the flux (2.29) similarly to the Teorell formula (1.7). For this purpose, let us collect under $\nabla$ the terms which represent the chemical potential in perfect media: $\mu=R T \ln c+\mu_{0}$. We assume that $T$ and $\mu_{0}$ are constant in space. With these conditions,

$$
J_{r i}=-\frac{l k}{R T} \gamma_{r i}\left(\prod_{q} c_{q}^{\alpha_{r q}^{\mathrm{I}}+\alpha_{r q}^{\mathrm{II}}}\right) \sum_{j}\left(\alpha_{r j}^{\mathrm{II}}-\alpha_{r j}^{\mathrm{I}}\right) \nabla \mu_{j}(x) \text {. }
$$

\subsubsection{Examples}

Let us illustrate application of formula (2.29) by several elementary examples.

First of all, Fick's law: $A^{\mathrm{I}} \rightarrow A^{\mathrm{II}}$ and $A^{\mathrm{II}} \rightarrow A^{\mathrm{I}}$. For this system, $\alpha^{\mathrm{I}}=1, \alpha^{\mathrm{II}}=0, \beta^{\mathrm{I}}=0$, $\beta^{\mathrm{II}}=1$ and $\gamma=-1$.

Formula (2.29) gives

$$
J=l k c \frac{-1}{c} \nabla c=-l k \nabla c .
$$

This is exactly the standard Fick law. The diffusion equation is $\partial_{t} c=d \Delta c$. Here and further in this subsection we use $d$ for $l k$.

Exchange of positions: $A^{\mathrm{I}}+B^{\mathrm{II}} \rightarrow A^{\mathrm{II}}+B^{\mathrm{I}}$ together with the space-inverted process $A^{\mathrm{II}}+$ $B^{\mathrm{I}} \rightarrow A^{\mathrm{I}}+B^{\mathrm{II}}$, which is the same as the reverse process. For this case, $\alpha_{A}^{\mathrm{I}}=1, \alpha_{B}^{\mathrm{I}}=0, \alpha_{A}^{\mathrm{II}}=0$, $\alpha_{B}^{\mathrm{II}}=1, \beta_{A}^{\mathrm{I}}=0, \beta_{B}^{\mathrm{I}}=1, \beta_{A}^{\mathrm{II}}=1, \beta_{B}^{\mathrm{II}}=0, \gamma_{A}=\beta_{A}^{\mathrm{I}}-\alpha_{A}^{\mathrm{I}}=-1$, and $\gamma_{B}=\beta_{B}^{\mathrm{I}}-\alpha_{B}^{\mathrm{I}}=1$. Due to (2.29),

$$
\begin{aligned}
& J_{A}=-d(-1) c_{A} c_{B}\left[\frac{-1}{c_{A}} \nabla c_{A}+\frac{1}{c_{B}} \nabla c_{B}\right]=-d\left(c_{B} \nabla c_{A}-c_{A} \nabla c_{B}\right), \\
& J_{B}=-d c_{A} c_{B}\left[\frac{-1}{c_{A}} \nabla c_{A}+\frac{1}{c_{B}} \nabla c_{B}\right]=d\left(c_{B} \nabla c_{A}-c_{A} \nabla c_{B}\right) .
\end{aligned}
$$


The diffusion equations are

$$
\partial_{t} c_{A}=d\left(c_{B} \Delta c_{A}-c_{A} \Delta c_{B}\right), \partial_{t} c_{B}=d\left(c_{A} \Delta c_{B}-c_{B} \Delta c_{A}\right) .
$$

Repulsion of components $A$ and $B$ ( $A$ is mobile). The mechanism is $A^{\mathrm{I}}+B^{\mathrm{I}} \rightarrow A^{\mathrm{II}}+B^{\mathrm{I}}$ together with the space-inverted process $A^{\mathrm{II}}+B^{\mathrm{II}} \rightarrow A^{\mathrm{I}}+B^{\mathrm{II}}$, which does not coincide with the reverse process. For this mechanism, $\alpha_{A}^{\mathrm{I}}=1, \alpha_{B}^{\mathrm{I}}=1, \alpha_{A}^{\mathrm{II}}=0, \alpha_{B}^{\mathrm{II}}=0, \beta_{A}^{\mathrm{I}}=0, \beta_{B}^{\mathrm{I}}=1, \beta_{A}^{\mathrm{II}}=1$, $\beta_{B}^{\mathrm{II}}=0, \gamma_{A}=\beta_{A}^{\mathrm{I}}-\alpha_{A}^{\mathrm{I}}=-1$, and $\gamma_{B}=\beta_{B}^{\mathrm{I}}-\alpha_{B}^{\mathrm{I}}=0$. Formula (2.29) gives:

$$
\begin{aligned}
J_{A} & =-d(-1) c_{A} c_{B}\left[\frac{-1}{c_{A}} \nabla c_{A}+\frac{-1}{c_{B}} \nabla c_{B}\right] \\
& =-d\left(c_{B} \nabla c_{A}+c_{A} \nabla c_{B}\right)=-d \nabla\left(c_{A} c_{B}\right), \\
J_{B} & =0 .
\end{aligned}
$$

We can see that $B$ activates diffusion of $A$ (the term $c_{B} \nabla c_{A}$ ) and, at the same time, pushes $A$ in the area with lower concentration of $B$ (the term $c_{A} \nabla c_{B}$ ).

The diffusion equation is

$$
\partial_{t} c_{A}=d \Delta\left(c_{A} c_{B}\right)
$$

The no-flux steady states of these diffusion equations are given by the condition: $c_{A} c_{B}=$ const.

If we assume that $B$ is also mobile by a similar mechanism then we get a system of equations (with two different diffusion coefficients):

$$
\begin{aligned}
& J_{A}=-d_{A} \nabla\left(c_{A} c_{B}\right), J_{B}=-d_{B} \nabla\left(c_{A} c_{B}\right), \\
& \partial_{t} c_{A}=d_{A} \Delta\left(c_{A} c_{B}\right), \partial_{t} c_{B}=d_{B} \Delta\left(c_{A} c_{B}\right) .
\end{aligned}
$$

Let us change variables:

$$
c_{+}=\frac{c_{A}}{d_{A}}+\frac{c_{B}}{d_{B}}, c_{-}=\frac{c_{A}}{d_{A}}-\frac{c_{B}}{d_{B}} .
$$

In these variables, $c_{A} c_{B}=\frac{d_{A} d_{B}}{4}\left(c_{+}^{2}-c_{-}^{2}\right), \partial_{t} c_{-}=0$ and for $c_{+}$we have the porous media equation:

$$
\partial_{t} c_{+}=\frac{d_{A} d_{B}}{2} \Delta c_{+}^{2}
$$

\subsection{Principle of Detailed Balance and Dissipation Inequality}

\subsubsection{Detailed balance and Coupling of Direct and Reverse Processes}

In this subsection, we formulate the principle of detailed balance for MAL diffusion. Physically, it follows from microreversibility.

For every elementary process,

$$
\sum_{i} \alpha_{r i}^{\mathrm{I}} A_{i}^{\mathrm{I}}+\sum_{i} \alpha_{r i}^{\mathrm{II}} A_{i}^{\mathrm{II}} \rightarrow \sum_{i} \beta_{r i}^{\mathrm{I}} A_{i}^{\mathrm{I}}+\sum_{i} \beta_{r i}^{\mathrm{II}} A_{i}^{\mathrm{II}},
$$


the reverse process is (just invert arrows):

$$
\sum_{i} \beta_{r i}^{\mathrm{I}} A_{i}^{\mathrm{I}}+\sum_{i} \beta_{r i}^{\mathrm{II}} A_{i}^{\mathrm{II}} \rightarrow \sum_{i} \alpha_{r i}^{\mathrm{I}} A_{i}^{\mathrm{I}}+\sum_{i} \alpha_{r i}^{\mathrm{II}} A_{i}^{\mathrm{II}} .
$$

Let us distinguish the quantities for the reverse and direct processes by the upper indices \pm . The simple algebraic relations hold:

$$
\alpha_{r i}^{\mathrm{I}, \mathrm{II} \mp}=\beta_{r i}^{\mathrm{I}, \mathrm{II} \pm} \text { and } \gamma_{r i}^{\mathrm{I}, \mathrm{II} \mp}=-\gamma_{r i}^{\mathrm{I}, \mathrm{II} \pm} .
$$

Therefore,

$$
\alpha_{r i}^{\mathrm{I}+}+\alpha_{r i}^{\mathrm{II}+}=\beta_{r i}^{\mathrm{I}-}+\beta_{r i}^{\mathrm{II}-}=\alpha_{r i}^{\mathrm{I}-}+\alpha_{r i}^{\mathrm{II}-}=\beta_{r i}^{\mathrm{I}+}+\beta_{r i}^{\mathrm{II}+} .
$$

The reaction rates are

$$
\begin{aligned}
& w_{r}^{+}\left(c^{\mathrm{I}}, c^{\mathrm{II}}\right)=k_{r}^{+} \prod_{i}\left(c_{i}^{\mathrm{I}}\right)^{\alpha_{r i}^{\mathrm{I}}} \prod_{i}\left(c_{i}^{\mathrm{II}}\right)^{\alpha_{r i}^{\mathrm{II}}}, \\
& w_{r}^{-}\left(c^{\mathrm{I}}, c^{\mathrm{II}}\right)=k_{r}^{-} \prod_{i}\left(c_{i}^{\mathrm{I}}\right)^{\beta_{r i}^{\mathrm{I}}} \prod_{i}\left(c_{i}^{\mathrm{II}}\right)^{\beta_{r i}^{\mathrm{II}}},
\end{aligned}
$$

Let there exist a uniform strictly positive point of detailed balance: such a strictly positive vector $c^{*}$ that for all $r$

$$
w_{r}^{+}\left(c^{*}, c^{*}\right)=w_{r}^{-}\left(c^{*}, c^{*}\right) .
$$

This means that

$$
k_{r}^{+} \prod_{i}\left(c_{i}^{*}\right)^{\alpha_{r i}^{\mathrm{I}}+\alpha_{r i}^{\mathrm{II}}}=k_{r}^{-} \prod_{i}\left(c_{i}^{*}\right)^{\beta_{r i}^{\mathrm{I}}+\beta_{r i}^{\mathrm{II}}} .
$$

Let us use the relations $\alpha_{r i}^{\mathrm{I}}+\alpha_{r i}^{\mathrm{II}}=\beta_{r i}^{\mathrm{I}}+\beta_{r i}^{\mathrm{II}}$. Therefore, the principle of detailed balance for MAL diffusion can be reformulated: for all $r$

$$
k_{r}^{+}=k_{r}^{-} .
$$

Let us join processes (2.37), (2.38) and write for them the diffusion flux analogously to (2.29):

$$
\begin{aligned}
J_{r i} & =-\gamma_{r i}\left[w_{r}^{+}(c(x), c(x+l))-w_{r}^{-}(c(x+l), c(x))\right] \\
& =-l \gamma_{r i} \sum_{j}\left(\left.\frac{\partial w_{r}^{+}\left(c^{\mathrm{I}}, c^{\mathrm{II}}\right)}{\partial c_{j}^{\mathrm{II}}}\right|_{c^{\mathrm{I}}=c^{\mathrm{II}}=c(x)}-\left.\frac{\partial w_{r}^{-}\left(c^{\mathrm{I}}, c^{\mathrm{II}}\right)}{\partial c_{j}^{\mathrm{I}}}\right|_{c^{\mathrm{I}}=c^{\mathrm{II}}=c(x)}\right) \nabla c_{j}(x) \\
& =-l \gamma_{r i} w_{r}(c(x), c(x)) \sum_{j} \frac{\gamma_{r j}}{c_{j}} \nabla c_{j}(x) \\
& =-l k \gamma_{r i}\left(\prod_{q} c_{q}^{\alpha_{r q}^{\mathrm{I}}+\alpha_{r q}^{\mathrm{II}}}\right) \sum_{j} \frac{\gamma_{r j}}{c_{j}} \nabla c_{j}(x) .
\end{aligned}
$$

According to this expression for the diffusion flux, (2.41), the matrix of diffusion coefficients for the elementary process (2.27) (together with its space-inverted process) is

$$
D_{i j}^{r}(c)=d_{r}\left(\prod_{q} c_{q}^{\alpha_{r q}^{\mathrm{I}}+\alpha_{r q}^{\mathrm{II}}}\right) \frac{\gamma_{r i} \gamma_{r j}}{c_{j}}
$$


where $d=\operatorname{const}\left(=k_{r} l\right)$.

This matrix is symmetric with respect to the inner product

$$
\langle a, b\rangle_{c}=\sum_{i} \frac{a_{i} b_{i}}{c_{i}} .
$$

This means that

$$
\langle D a, b\rangle_{c}=\langle a, D b\rangle_{c}
$$

or in coordinates

$$
\sum_{i j} \frac{D_{i j} a_{j} b_{i}}{c_{i}}=\sum_{i j} \frac{a_{i} D_{i j} b_{j}}{c_{i}} .
$$

Indeed, let $\widetilde{w}_{r}=d_{r}\left(\prod_{q} c_{q}^{\alpha_{r q}^{\mathrm{I}}+\alpha_{r q}^{\mathrm{II}}}\right)$. For two scalar products, $\langle D a, b\rangle_{c}$ and $\langle a, D b\rangle_{c}$ we get

$$
\langle D a, b\rangle_{c}=\sum_{i j} \frac{D_{i j} a_{j} b_{i}}{c_{i}}=\widetilde{w}_{r} \sum_{i j} \frac{\gamma_{r i} \gamma_{r j} a_{j} b_{i}}{c_{j} c_{i}}
$$

and

$$
\langle a, D b\rangle_{c}=\sum_{i j} \frac{a_{i} D_{i j} b_{j}}{c_{i}}=\widetilde{w}_{r} \sum_{i j} \frac{a_{i} \gamma_{r i} \gamma_{r j} b_{j}}{c_{i} c_{j}},
$$

These two expressions differ only in the notation of dummy indexes, $i$ and $j$. Therefore, $D$ is symmetric with respect to the inner product (2.43).

$D$ is also positive semi-definite. Indeed, for any vector $\xi$,

$$
\left.\langle\xi, D \xi\rangle=\sum_{i} \frac{\xi_{i} D_{i j}^{r} \xi_{j}}{c_{i}}=\widetilde{w}_{r}\langle\xi, \gamma)^{2} \geq 0\right\rangle .
$$

This expression may be zero at a positive state $\left(c_{i}>0\right)$ if and only if the vector $\xi$ is orthogonal to the vector $\gamma_{r}$ in the inner product (2.43). If we summarize diffusion coefficients for all pairs of mutually inverse elementary processes then we get

$$
D_{i j}=\sum_{r} D_{i j}^{r}(c)=\sum_{r} \widetilde{w}_{r} \frac{\gamma_{r i} \gamma_{r j}}{c_{j}}
$$

For this $D_{i j}$,

$$
\sum \frac{\xi_{i} D_{i j} \xi_{j}}{c_{i}} \geq 0
$$

and is zero if and only if vector $\xi$ is orthogonal to all vectors $\gamma_{r}$ in the inner product (2.43).

The corresponding diffusion equations have the divergent form:

$$
\frac{\partial c}{\partial t}=\operatorname{div}(D(c) \nabla c) .
$$


It might be useful to represent the flux (2.41) similarly to the Teorell formula (1.7). For this purpose, let us collect under $\nabla$ the terms which represent the chemical potential in perfect media: $\mu=R T \ln c+\mu_{0}$. We assume that $T$ and $\mu_{0}$ are constant in space. With these conditions,

$$
J_{r i}=-\frac{l k}{R T} \gamma_{r i}\left(\prod_{q} c_{q}^{\alpha_{r q}^{\mathrm{I}}+\alpha_{r q}^{\mathrm{II}}}\right) \sum_{j} \gamma_{r j} \nabla \mu_{j}(x) .
$$

The difference from the MAL Teorell formula (2.32) is obvious: for the systems with detailed balance (2.46) the matrix of the coefficients in the Teorell formula is symmetric for each elementary process together with its reverse process because it is a product of a number (in square brackets) and the symmetric matrix $\gamma_{r i} \gamma_{r j}$ :

$$
\left[\frac{l k}{R T}\left(\prod_{q} c_{q}^{\alpha_{r q}^{\mathrm{I}}+\alpha_{r q}^{\mathrm{II}}}\right)\right] \gamma_{r i} \gamma_{r j}
$$

For the general MAL diffusion (with detailed symmetry in space) the matrix these coefficients for the elementary process together with its space-inverted process is not symmetric in general: it is a scalar multiple of the matrix

$$
\gamma_{r i}\left(\alpha_{r j}^{\mathrm{II}}-\alpha_{r j}^{\mathrm{I}}\right) \text {. }
$$

Its symmetry may be guaranteed if the space inversion of the elementary process coincides with its reversion in time (i.e. $\alpha_{r}^{\mathrm{II}}$ coincides with $\beta_{r}^{\mathrm{I}}$ ).

For MAL chemical kinetics, there is a sufficient algebraic condition for detailed balance that is independent of the microreversibility and follows just from the stoichiometric equations. Indeed, let us assume that all the reactions are reversible and all the stoichiometric vectors $\gamma_{r}$ in (2.7) are linearly independent. Then, at the equilibrium, from the condition $\dot{c}=\sum_{r} w_{r} \gamma_{r}=0$ we get $w_{r}=0$ for all $r$. That is the detailed balance condition.

For MAL diffusion we have also a specific ("diffusion") algebraic condition: if for all elementary processes the space-inverted reaction is the reverse reaction then the principle of detailed balance follows from the space symmetry condition. For such systems, the coupling "process-space inverted process" coincides with the coupling "process-reverse process" and equations (2.41) coincide with (2.29).

For an elementary process (2.37), let the space-inverted process not coincide with the reverse process. With the condition of detailed balance, it is straightforward to check that for the pair of elementary processes $(2.37),(2.38)$, the space-inverted process to (2.37) and the reverse process to this space-inverted one generate the same diffusion equations and the diffusion coefficient as the original pair does. These two couples together produce the flux that is twice as large as (2.41).

\subsubsection{The Dissipation Inequality and Detailed Balance}

In this subsection, we construct the Lyapunov functional for the general MAL diffusion models and calculate its time derivative due to the diffusion equations. 
Let us select any strictly positive reference concentration vector $c^{*}$ and take (2.8)

$$
G=\sum_{i} c_{i}\left(\ln \left(\frac{c_{i}}{c_{i}^{*}}\right)-1\right)+\sum_{i} c_{i}^{*} .
$$

Let us consider this system in a bounded domain $V$ with smooth boundary and with zero fluxes through its boundary: $(n, J)=0$ at any point of $\partial V$ at any time ( $n$ is the vector of the outer normal). Due to the definition of flux (2.29), it is sufficient that $\left(n, \operatorname{grad} c_{j}\right)=0$ on $\partial V$ for all $j$ (but it is not necessary).

The Lyapunov functional is

$$
\mathbf{G}=\sum_{i} \int_{V}\left[c_{i}\left(\ln \left(\frac{c_{i}}{c_{i}^{*}}\right)-1\right)+\sum_{i} c_{i}^{*}\right] \mathrm{d} x .
$$

Due to the boundary conditions and the Gauss-Ostrogradskii theorem

$$
\frac{\mathrm{d} \mathbf{G}}{\mathrm{d} t}=-\sum_{i} \int_{V} \ln \left(\frac{c_{i}}{c_{i}^{*}}\right) \operatorname{div} J_{i} \mathrm{~d} x=\sum_{i} \int_{V}\left(\nabla_{x}\left(\ln \frac{c_{i}}{c_{i}^{*}}\right), J_{i}\right) \mathrm{d} x .
$$

Let us assume the principle of detailed balance and calculate $\dot{\mathbf{G}}(2.49)$ due to diffusion equation with the matrix of diffusion coefficients (2.44):

$$
\begin{aligned}
\frac{\mathrm{d} \mathbf{G}}{\mathrm{d} t} & =\sum_{i} \int_{V}\left(\nabla_{x}\left(\ln \frac{c_{i}}{c_{i}^{*}}\right), J_{i}\right) \mathrm{d} x \\
& =-\sum_{r} \int_{V} \widetilde{w}_{r} \sum_{i j}\left(\nabla_{x}\left(\ln c_{i}\right), \gamma_{r i} \gamma_{r j} \nabla_{x}\left(\ln c_{j}\right)\right) \mathrm{d} x \\
& =-\sum_{r} \int_{V} \widetilde{w}_{r}\left(\nabla_{x}\left(\sum_{i} \ln \left(\gamma_{r i} c_{i}\right)\right), \nabla_{x}\left(\sum_{j} \ln \left(\gamma_{r j} c_{j}\right)\right)\right) \mathrm{d} x \\
& =-\sum_{r} \int_{V} \widetilde{w}_{r}\left(\nabla_{x}\left(\gamma_{r}, \nabla_{c} G\right)\right)^{2} \mathrm{~d} x \leq 0 .
\end{aligned}
$$

Here, $($,$) is the standard Euclidean inner product both in space and in the concentration space.$

As we can see from this dissipation inequality, the only positive equilibria for diffusion equations with detailed balance conditions satisfy the conditions $\left(\gamma_{r}, \nabla_{c} G\right)=$ const for all $r$. Another form of these conditions is

$$
\prod_{i} c_{i}^{\gamma_{r i}}=\text { const }
$$

Inequality (2.50) demonstrates a significant difference between the two classes of diffusion mechanism. If the stoichiometric vectors $\gamma_{r}$ form a basis in the concentration space then all the equilibria are uniform because in this case the condition $\left(\gamma_{r}, \nabla_{c} G\right)=$ const (for all $r$ ) implies $\nabla_{c} G=$ const, that is, $\ln c_{i}=$ const for all $i$, hence, $c_{i}=$ const for all $i$. Let us call this mechanism 
the mechanisms with mixing. The first example is Fick's mechanism: if the diffusion constant is not zero for all components then all the equilibria of the system are uniform.

If $\operatorname{span}\left\{\gamma_{r}\right\}$ does not coincide with the concentration space then there exist the invariants of diffusion. They are given by the linear functionals that annihilate all the vectors $\gamma_{r}$. For example, in diffusion by jumps on the free places (Fig. 3(b)) the value of the sum $c_{Z}(x)+\sum_{i} c_{i}(x)$ is locally conserved. In the mechanism, $A^{\mathrm{I}}+B^{\mathrm{I}} \rightarrow A^{\mathrm{II}}+B^{\mathrm{I}}, A^{\mathrm{II}}+B^{\mathrm{II}} \rightarrow A^{\mathrm{I}}+B^{\mathrm{II}}$, concentration of $B$ is conserved. These locally conserved quantities together with the condition of positivity $c_{i} \geq 0$ define a convex body where the vector of concentrations may be situated at a given point. This body depends on the values of the conserved quantities, differs for different points, but does not change in time.

\subsection{Complex Balance in MAL Diffusion}

\subsubsection{Complex Balance Conditions for MAL Diffusion}

The complex balance condition does not assume any space or time symmetry. The only microscopic assumption is the Markov fast microscopic kinetic with relatively small amount of active intermediate complexes [97, 54].

We discussed this condition for the MAL kinetics in Section 2.1.4., and now let us transform it into a condition for the MAL diffusion equation. First of all, we should abandon the symmetry conditions $k^{\prime}=k$ (space symmetry) and $k^{+}=k^{-}$(microreversibility). Without these conditions, the zero-order terms in the expression for fluxes will not be annihilated by balance between the elementary processes with given pair of vectors $\left(\alpha_{r}^{\mathrm{I}}, \alpha_{r}^{\mathrm{II}}\right)$ and the processes with the same pair $\left(\beta_{r}^{\mathrm{I}}, \beta_{r}^{\mathrm{II}}\right)(2.20)$.

Let the stoichiometric mechanism of the diffusion be given:

$$
\sum_{i} \alpha_{r i}^{\mathrm{I}} A_{i}^{\mathrm{I}}+\sum_{i} \alpha_{r i}^{\mathrm{II}} A_{i}^{\mathrm{II}} \rightarrow \sum_{i} \beta_{r i}^{\mathrm{I}} A_{i}^{\mathrm{I}}+\sum_{i} \beta_{r i}^{\mathrm{II}} A_{i}^{\mathrm{II}}
$$

where all the elementary processes have different numbers $r$ and the space-inverted and reverse processes are represented separately. Let us consider all pairs of vectors, $\left(\alpha_{r}^{\mathrm{I}}, \alpha_{r}^{\mathrm{II}}\right)$ and $\left(\beta_{r}^{\mathrm{I}}, \beta_{r}^{\mathrm{II}}\right)$. Let us enumerate all the different pairs: $y_{1}, y_{2}, \ldots, y_{q}=\left(y_{q}^{\mathrm{I}}, y_{q}^{\mathrm{II}}\right)$. For each $y_{q}$ there are two sets of reactions, $R_{q}^{+}, R_{q}^{-}$:

$$
R_{q}^{+}=\left\{r \mid\left(\alpha_{r}^{\mathrm{I}}, \alpha_{r}^{\mathrm{II}}\right)=y_{q}\right\}, R_{q}^{-}=\left\{r \mid\left(\beta_{r}^{\mathrm{I}}, \beta_{r}^{\mathrm{II}}\right)=y_{q}\right\} .
$$

The complex balance condition is: there exists a strictly positive vector $c^{*}$ such that

$$
\sum_{r \in R_{q}^{+}} w_{r}\left(c^{*}, c^{*}\right)=\sum_{r \in R_{q}^{-}} w_{r}\left(c^{*}, c^{*}\right) \text { for all } q .
$$

For MAL this means

$$
\sum_{r \in R_{q}^{+}} k_{r} \prod_{i}\left(c_{i}^{*}\right)^{\alpha_{r}^{\mathrm{I}}+\alpha_{r}^{\mathrm{II}}}=\sum_{r \in R_{l}^{-}} k_{q} \prod_{i}\left(c_{i}^{*}\right)^{\alpha_{q}^{\mathrm{I}}+\alpha_{q}^{\mathrm{II}}} \text { for all } l .
$$


For $r \in R_{q}^{+}$,

$$
\alpha_{r}^{\mathrm{I}}+\alpha_{r}^{\mathrm{II}}=y_{q}^{\mathrm{I}}+y_{q}^{\mathrm{II}},
$$

and for $r \in R_{l}^{-}$

$$
\beta_{r}^{\mathrm{I}}+\beta_{r}^{\mathrm{II}}=y_{q}^{\mathrm{I}}+y_{q}^{\mathrm{II}} .
$$

Therefore, all the monomials coincide in the right and the left hand sides of (2.53) for the given $q$ and we can write the complex balance condition for diffusion in the following form:

$$
\sum_{r \in R_{q}^{+}} k_{r}=\sum_{r \in R_{q}^{-}} k_{r} \text { for all } q .
$$

Let us calculate the flux

$$
J=\sum_{r} \gamma_{r} w_{r}(c(x), c(x+l))
$$

at first order in $l$. We group terms in this sum and each group will corresponds to a pair $y_{q}$. For each elementary process with number $r$ there are two $q, q_{\alpha}(r)$ and $q_{\beta}(r): r \in R_{q_{\alpha}(r)}^{+}$and $r \in R_{q_{\beta}(r)}^{-}$. We split the term $\gamma_{r} w_{r}(c(x), c(x+l))$ in two terms:

$$
\gamma_{r} w_{r}(c(x), c(x+l))=\beta_{r} w_{r}(c(x), c(x+l))-\alpha_{r} w_{r}(c(x), c(x+l))
$$

We associate the first of them with $y_{q_{\beta}(r)}$ and the second with $y_{q_{\alpha}(r)}$. The result is represented below:

$$
\begin{aligned}
J= & -\sum_{r} \gamma_{r} w_{r}(c(x), c(x+l)) \\
= & \sum_{q}\left(\sum_{r \in R_{q}^{+}} \alpha_{r}^{\mathrm{I}} w_{r}(c(x), c(x+l))-\sum_{r \in R_{q}^{-}} \beta_{r}^{\mathrm{I}} w_{r}(c(x), c(x+l))\right) \\
= & \sum_{q} y_{q}^{\mathrm{I}}\left(\sum_{r \in R_{q}^{+}} w_{r}(c(x), c(x+l))-\sum_{r \in R_{q}^{-}} w_{r}(c(x), c(x+l))\right) \\
= & \sum_{q} y_{q}^{\mathrm{I}} \prod_{i} c_{i}^{y_{q}^{\mathrm{I}}+y_{q}^{\mathrm{II}}}\left(\sum_{r \in R_{q}^{+}} k_{r}-\sum_{r \in R_{q}^{-}} k_{r}\right) \\
& +l \sum_{q} y_{q}^{\mathrm{I}} \prod_{i} c_{i}^{y_{q}^{\mathrm{I}}+y_{q}^{\mathrm{II}}}\left(\sum_{r \in R_{q}^{+}} k_{r} \sum_{j} \frac{\alpha_{r j}^{\mathrm{II}}}{c_{j}} \nabla c_{j}-\sum_{r \in R_{q}^{-}} k_{r} \sum_{j} \frac{\alpha_{r j}^{\mathrm{II}}}{c_{j}} \nabla c_{j}\right)+o(l) .
\end{aligned}
$$

The zero-order term is zero because of the complex balance condition (2.54). Let us take into account that both for $r \in R_{q}^{+}$and $r \in R_{q}^{-}$

$$
\alpha^{\mathrm{I}}+\alpha^{\mathrm{II}}=y_{q}^{\mathrm{I}}+y_{q}^{\mathrm{II}} .
$$


Therefore, if for two $y_{q}, y_{s}$

$$
R_{q}^{+} \bigcap R_{s}^{-} \neq \emptyset
$$

then

$$
y_{q}^{\mathrm{I}}+y_{q}^{\mathrm{II}}=y_{q}^{\mathrm{I}}+y_{q}^{\mathrm{II}} .
$$

For the set of all vectors $y_{q}^{\mathrm{I}}+y_{q}^{\mathrm{II}}$ we use notation $Z$. For each $z \in Z$

$$
Y_{z}=\left\{y_{q} \mid y_{q}^{\mathrm{I}}+y_{q}^{\mathrm{II}}=z\right\}
$$

Let us group the terms with the same vectors $z=y_{q}^{\mathrm{I}}+y_{q}^{\mathrm{II}} \in Z$ together and write the expression for the flux for the first order:

$$
\begin{aligned}
& J=l \sum_{z \in Z} \prod_{i} c_{i}^{z_{i}} J^{z}, \\
& J^{z}=\sum_{y_{q} \in Y_{z}} y_{q}^{\mathrm{I}} \sum_{j}\left(\sum_{r \in R_{q}^{+}} k_{r} \frac{\alpha_{r j}^{\mathrm{II}}}{c_{j}}-\sum_{r \in R_{q}^{-}} k_{r} \frac{\alpha_{r j}^{\mathrm{II}}}{c_{j}}\right) \nabla c_{j} .
\end{aligned}
$$

Let us study the expression for $J^{z}$ for given $z$. First of all,

$$
\sum_{y_{q} \in Y_{z}} \sum_{j}\left(\sum_{r \in R_{q}^{+}} k_{r} \frac{\alpha_{r j}^{\mathrm{II}}}{c_{j}}-\sum_{r \in R_{q}^{-}} k_{r} \frac{\alpha_{r j}^{\mathrm{II}}}{c_{j}}\right) \nabla c_{j}=0
$$

because each $k_{r}$ from this sum enters it twice, with the opposite signs, one time as an element of $R_{q}^{+}$for some $y_{q} \in Y_{z}$ (with + ) and the second time (with - ) as an element of $R_{s}^{-}$for another $y_{s} \in Y_{z}$ with the same sum

$$
y_{s}^{\mathrm{I}}+y_{s}^{\mathrm{II}}=y_{q}^{\mathrm{I}}+y_{q}^{\mathrm{II}}=z .
$$
for $J^{z}$ :

We note that $y_{q}^{\mathrm{I}}=z-y_{q}^{\mathrm{II}}$ for $y_{q} \in Y_{z}$ in the sum (2.56). This allows us to rewrite the expression

$$
J^{z}=-\sum_{y_{q} \in Y_{z}} y_{q}^{\mathrm{II}} \sum_{j}\left(\sum_{r \in R_{q}^{+}} k_{r} \frac{\alpha_{r j}^{\mathrm{II}}}{c_{j}}-\sum_{r \in R_{q}^{-}} k_{r} \frac{\alpha_{r j}^{\mathrm{II}}}{c_{j}}\right) \nabla c_{j} .
$$

\subsubsection{The Dissipation Inequality and Complex Balance for MAL Diffusion}

We would like to demonstrate some similarity of the expression for $J^{z}$ and some formulas from the theory of Markov chains. Vectors $y_{q} \in Y_{z}$ numerate the states. Elementary processes correspond to transitions between states. Each nonzero constant $k_{r}$ corresponds to two vectors $y_{q}, y_{s} \in Y_{z}$ : $r \in R_{q}^{+}$and $r \in R_{s}^{-}$. We substitute the index $r$ by two indexes $q, s$ and use notation $k_{s q}$ (or even $k_{s \leftarrow q}$ ). If there is no nonzero constant for this pair $q, s$ then we take $k_{s \leftarrow q}=0$. In particular, $k_{q q}=0$. The complex balance condition $(2.54)$ reads:

$$
\sum_{q} k_{s q}=\sum_{q} k_{q s}
$$


This means that the constants $k_{s q}$ describe a continuous time Markov chain with the Master equation

$$
\dot{\pi}_{q}=\sum_{s}\left(k_{q s} \pi_{s}-k_{s q} \pi_{q}\right)
$$

and equidistribution in equilibrium. Here $\pi_{q}$ is the probability to find the system in the state $q$ and $k_{q s} \pi_{s}$ is the probability flux from the state $s$ to the state $q$. We can use the steady state condition (2.58) and rewrite the Master equation (2.59):

$$
\dot{\pi}_{q}=\sum_{s} k_{q s}\left(\pi_{s}-\pi_{q}\right) .
$$

From this form, it is easy to see that the functional

$$
H=\frac{1}{2} \sum_{q} \pi_{q}^{2}
$$

monotonically decreases due to the system dynamics:

$$
\frac{\mathrm{d} H}{\mathrm{~d} t}=\sum_{s q} \pi_{q} k_{q s}\left(\pi_{s}-\pi_{q}\right) \leq 0 .
$$

To prove (2.61), let us use the identity

$$
\sum_{q} \dot{\pi}_{q}=\sum_{s}\left(k_{q s} \pi_{s}-k_{s q} \pi_{q}\right)=\sum_{s} k_{q s}\left(\pi_{s}-\pi_{q}\right)=0 .
$$

This condition holds for all values of numbers $\pi_{q}$ (this is obvious for the Master equation in the form (2.59)).

In particular,

$$
\sum_{s} k_{q s}\left(\frac{1}{2} \pi_{q}^{2}-\frac{1}{2} \pi_{s}^{2}\right)=0 .
$$

Let us add this expression to the right hand side of (2.61):

$$
\frac{\mathrm{d} H}{\mathrm{~d} t}=\sum_{s q} k_{q s}\left(\frac{1}{2} \pi_{q}^{2}-\frac{1}{2} \pi_{s}^{2}+\pi_{q}\left(\pi_{s}-\pi_{q}\right)\right) \leq 0
$$

because $k_{q s} \geq 0$ and the expression in the parentheses is non-positive:

$$
\frac{1}{2} \pi_{q}^{2}-\frac{1}{2} \pi_{s}^{2}+\pi_{q}\left(\pi_{s}-\pi_{q}\right)=-\frac{1}{2}\left(\pi_{q}-\pi_{s}\right)^{2} \leq 0
$$

Therefore, we have proved the inequality for our set of coefficients $k_{q s}$ :

$$
\sum_{s q} \pi_{q}\left(k_{q s} \pi_{s}-k_{s q} \pi_{q}\right)=\sum_{s q} \pi_{q} k_{q s}\left(\pi_{s}-\pi_{q}\right) \leq 0
$$


for any set of numbers $\pi_{q}$. This inequality means that $\mathrm{d} H / \mathrm{d} t \leq 0$ (2.61). It is zero if all $\pi_{q}$ coincide $\left(\pi_{s}=\pi_{q}\right.$ for all $\left.s, q\right)$.

For our purposes, it is important to know when the zero time derivative of $H(\mathrm{~d} H / \mathrm{d} t=0)$ is equivalent to the equidistribution $\left(\pi_{s}=\pi_{q}\right.$ for all $\left.s, q\right)$. They are equivalent if the Markov chain (2.59) is ergodic. The conditions of ergodicity are well known $[95,106]$ : the chain $(2.59)$ is ergodic if for any two $s, q(s \neq q)$ there exists a oriented path from $s$ to $q$ in the graph of the network, that is such a sequence

$$
r_{0}, r_{1}, \ldots, r_{g} \text { that } s=r_{0}, q=r_{g} \text { and } k_{r_{j+1} r_{j}}>0 \text { fo all } j=0, \ldots, g .
$$

This means that the graph of of transitions of the Markov chain (2.59) is strongly connected.

To apply this inequality to the proof of the dissipation inequality, we have to rewrite the expression for $J^{z}(2.57)$ using these notations, $k_{q s}$ instead of $k_{r}$ :

$$
J^{z}=\sum_{y_{q} \in Y_{z}} y_{q}^{\mathrm{II}} \sum_{j} \sum_{s}\left(k_{q s} y_{s j}^{\mathrm{II}}-k_{s q} y_{q j}^{\mathrm{II}}\right) \nabla_{x} \ln c_{j} .
$$

Now we are in the position to prove the dissipation inequality for MAL diffusion equation with complex balance. In a bounded domain $V$ with smooth boundary and without fluxes through boundary we have to estimate $\dot{\mathbf{G}}$ (2.48), (2.49).

$$
\begin{aligned}
\frac{\mathrm{d} \mathbf{G}}{\mathrm{d} t} & =-\int_{V} \sum_{j} \ln \left(\frac{c_{j}}{c_{j}^{*}}\right) \operatorname{div} J_{j} \mathrm{~d} x \\
& =\int_{V} \sum_{j}\left(\nabla_{x} \ln c_{j}, J_{j}\right) \mathrm{d} x \\
& =l \sum_{z \in Z} \int_{V} \prod_{i} c_{i}^{z_{i}} \sum_{j}\left(\nabla_{x} \ln c_{j}, J_{j}^{z}\right) \mathrm{d} x .
\end{aligned}
$$

Due to the representation of $J^{z}(2.65)$,

$$
\sum_{j}\left(\nabla_{x} \ln c_{j}, J_{j}^{z}\right)=\sum_{q s}\left(\pi_{q},\left(k_{q s} \pi_{s}-k_{s q} \pi_{q}\right)\right) \leq 0,
$$

where $\pi_{q}$ is a space vector: $\pi_{q}=\sum_{i} y_{q i}^{\mathrm{II}} \nabla_{x} \ln c_{i}$ and for $y_{q}, y_{s}$

$$
y_{q, s}^{\mathrm{I}}+y_{q, s}^{\mathrm{II}}=z
$$

This expression has exactly the form (2.64) for each space coordinate. Finally,

$$
\sum_{j}\left(\nabla_{x} \ln c_{j}, J_{j}^{z}\right) \leq 0
$$

and it is zero if all $\pi_{q}=\sum_{i} y_{q i}^{\mathrm{II}} \nabla_{x} \ln c_{i}$ coincide. 
The reverse statement,

$$
\text { all } \pi_{q}=\sum_{i} y_{q i}^{\mathrm{II}} \nabla_{x} \ln c_{i} \text { coincide if } \sum_{j}\left(\nabla_{x} \ln c_{j}, J_{j}^{z}\right)=0,
$$

is true if the auxiliary Markov chain is ergodic for given $z$ (i.e. the graph of transitions is strongly connected). Let us assume this ergodicity. For every $z$ we can define a linear subspace $E_{z}$ in the concentration space given by the system of equation

$$
E_{z}=\left\{e \mid\left(y_{q i}^{\mathrm{II}}, e\right) \text { coincide for all } y_{q} \in Y_{z}\right\}
$$

If

$$
\bigcap_{z \in Z} E_{z}=\{0\}
$$

then all the equilibria for this mechanism of diffusion are uniform. In particular, they are uniform if at least one $y_{q i}^{\mathrm{II}}=0$.

\subsection{Intermediate Summary}

We presented the formal language of the stoichiometric mechanism for description of nonlinear diffusion (2.21), (2.27), (2.37), (2.38). The general construction of the diffusion equations under various conditions is given:

- For systems with symmetry with respect to inversion in space $\left(k_{r}=k_{r}^{\prime}\right)(2.29),(2.30)$;

- For systems with microreversibility $\left(k_{r}^{+}=k_{r}-\right)$ (2.41), (2.42);

- For general systems with Markov microkinetics, which satisfy the complex balance conditions (2.54), (2.56).

For systems with detailed balance (microreversibility) and with complex balance (Markov microkinetics) the explicit formula for the free energy-type Lyapunov functional $G$ (2.48) is

$$
\mathbf{G}=\sum_{i} \int_{V}\left[c_{i}\left(\ln \left(\frac{c_{i}}{c_{i}^{*}}\right)-1\right)+\sum_{i} c_{i}^{*}\right] \mathrm{d} x .
$$

We found that $\dot{\mathrm{G}} \leq 0$ for the systems with detailed or complex balance (2.49), (2.66). These inequalities guarantee the thermodynamic behavior of diffusion. The detailed symmetry with respect to the inversion in space is insufficient for such an inequality and the diffusion collapse for them is possible.

The Mass Action Law by itself does not imply thermodynamics. In that sense, it is too flexible and needs additional requirements to respect the basic physics. This redundancy of MAL allows, at the same time, to use them in many other areas. The famous Lotka and Volterra models in Mathematical ecology [73, 110] are implementations of MAL for description of surviving and 
reproduction of animals, that is far from the initial area of MAL applications. Creation of mathematical genetics [33] and analysis of dynamical aspects of biological evolution [38] also use MAL in their backgrounds. Combination of MAL kinetics with nonlinear diffusion is very important for analysis of biological invasions and other phenomena in ecology [57, 61, 96, 89]. The MAL for diffusion can also generate equations that have no direct physical sense but may be used for modeling of some phenomena of non-physical nature.

On the other hand, in this approach, we did not take into account some basic physical properties, namely, the momentum and the center of mass conservation. The diffusion transport should be coupled with the viscous transport or elastic deformation (or both) because two reasons:

- The mass average velocity of diffusion

$$
u=\frac{\sum_{i} m_{i} J_{i}}{\sum_{i} m_{i}}
$$

where $m_{i}$ is the molecular mass of the $A_{i}$ particles, is, in general, not zero;

- The change of the mixture composition implies the change of pressure and, hence, the viscous flux or the elastic deformation (or both).

The careful analysis of these effects should give, for example a theory of the Kirkendall effect. In 1942, Kirkendall demonstrated experimentally that different atoms can migrate at different rates in an alloy, and this diffusion is accompanied by measurable local volume change and displacement of interfaces $[67,81]$. The kinetic theory of this effect is still under development and there remain open problems and new ideas are needed [82].

We return to the problem of correct description of the general transport equation in next Section.

The MAL formalism for diffusion is a flexible and effective tool for modeling. The semidiscrete MAL model may be used for numerical modeling directly as a sort of finite elements. Their coarse-graining and refinement should follow the main rule $k l=d$ where $l$ is the cell size, $k$ is a kinetic constant for the finite elements, $d$ is the invariant diffusion coefficient. For unstable processes, these provide a biharmonic regularization. These kinetic finite elements respect the basic physical properties like positivity of concentration, conservation laws and the second law of thermodynamics (under the relevant conditions of detailed or complex balance).

\section{Generalized Mass Action Law for Diffusion}

\subsection{Free Energy, Free Entropy, Chemical Potentials, Activities, and Generalized Mass Action Law}

\subsubsection{Thermodynamic Potentials}

In this Subsection, we present the thermodynamic approach to the generalized MAL. Exactly as in Section 2.1. we start from the chemical kinetic equations and then extend our approach to the transport processes. 
In the thermodynamic approach, the kinetic description of the multicomponent system requires the following inputs

1. A list of components;

2. A thermodynamic potential;

3. A list of elementary reactions represented by their stoichiometric equations;

4. A set of reaction rate constants.

Exactly as it was in Section 2.1., the list of components is just a set of symbols (the component names). We usually assume that this set is finite, $A_{1}, A_{2}, \ldots, A_{n}$. The definitions of stoichiometric equation and the corresponding vectors $\alpha_{r i}, \beta_{r i}$ are also the same.

There are many thermodynamic potentials and they form two series: energy and free energies and, on another hand, entropy and free entropies (the Massieu-Planck functions). Each of them has its own "natural variables" and if one of them is given in the natural variables then all other thermodynamic functions can be produced.

The names and standard notations of variables are:

- Internal energy, $U$;

- Entropy, $S$;

- Enthalpy, $H$;

- Temperature, $T$;

- Volume, $V$;

- Pressure, $P$;

- Number of particles (or moles) composing the $i$ th component $A_{i}, N_{i}$ ( $N$ is vector with coordinates $N_{i}$, the vector of composition);

- Chemical potential of the $i$ th component $A_{i}, \mu_{i}$.

The first potential in the energetic series is the internal energy $U(S, V, N)$ in the natural coordinates $S, V, N$ and

$$
\mathrm{d} U=T \mathrm{~d} S-P \mathrm{~d} V+\sum_{i} \mu_{i} \mathrm{~d} N_{i}
$$

The enthalpy, $H(S, P, N)=U+P V$ has the natural coordinates $S, P, N$ and

$$
\mathrm{d} H=T \mathrm{~d} S+V \mathrm{~d} P+\sum_{i} \mu_{i} \mathrm{~d} N_{i}
$$


The free energy (the Helmholtz energy), $F(T, V, N)=U-T S$ and

$$
\mathrm{d} F=-S \mathrm{~d} T-P \mathrm{~d} V+\sum_{i} \mu_{i} \mathrm{~d} N_{i}
$$

The free enthalpy (the Gibbs energy), $G(T, P, N)=H-T S$ and

$$
\mathrm{d} G=-S \mathrm{~d} T+V \mathrm{~d} P+\sum_{i} \mu_{i} \mathrm{~d} N_{i}
$$

The grand potential, $\Omega(T, V, \mu)=U-T S-\sum_{i} \mu_{i} N_{i}$ and

$$
\mathrm{d} \Omega=-S \mathrm{~d} T-P \mathrm{~d} V-\sum_{i} N_{i} \mathrm{~d} \mu_{i}
$$

The entropic series starts from the entropy $S(U, V, N)$ and

$$
\mathrm{d} S=\frac{1}{T} \mathrm{~d} U+\frac{P}{T} \mathrm{~d} V-\sum_{i} \frac{\mu_{i}}{T} \mathrm{~d} N_{i} .
$$

Therefore, the main set of the intensive variables for the entropic series is

$$
\frac{1}{T}=\frac{\partial S(U, V, N)}{\partial U}, \frac{P}{T}=\frac{\partial S(U, V, N)}{\partial V},-\frac{\mu_{i}}{T}=\frac{\partial S(U, V, N)}{\partial N_{i}} .
$$

The Massieu function, $\Phi\left(T^{-1}, V, N\right)=S-T^{-1} U(=-F / T)$ and

$$
\mathrm{d} \Phi=-U \mathrm{~d}\left(\frac{1}{T}\right)+\frac{P}{T} \mathrm{~d} V-\sum_{i} \frac{\mu_{i}}{T} \mathrm{~d} N_{i} .
$$

The Planck function, $\Xi\left(T^{-1}, T^{-1} P, N\right)=S-T^{-1} U-T^{-1} P V(=-G / T)$ and

$$
\mathrm{d} \Phi=-U \mathrm{~d}\left(\frac{1}{T}\right)-V \mathrm{~d}\left(\frac{P}{T}\right)-\sum_{i} \frac{\mu_{i}}{T} \mathrm{~d} N_{i} .
$$

All these functions are used for the definition of equilibrium. The main definition for an isolated system follows the R. Clausius two main laws formulated in 1865 [17]:

1. The energy of the Universe is constant.

2. The entropy of the Universe tends to a maximum.

More precisely, the entropy of the isolated system tends to a maximum under given $U, V$ and values of other conservation laws. Let the conservation laws be given:

$$
B_{j}=\sum_{i} b_{j i} N_{j},
$$


then the equilibrium is the maximizer of the entropy under given values of $U, V$ and $B_{j}$ :

$$
S(U, V, N) \rightarrow \max \text { subject to given } U, V \text { and }\left\{B_{j}\right\} .
$$

Gibbs [39] paid much attention to the dual formulation of this condition:

$$
U(S, V, N) \rightarrow \text { min subject to given } S, V \text { and }\left\{B_{j}\right\} .
$$

Other definitions of the same equilibrium are available through the free energies and entropies. For the free energies this is condition of minimum. Analogously to (3.2) we get

$$
\begin{aligned}
& H(S, P, N) \rightarrow \text { min subject to given } S, P \text { and }\left\{B_{j}\right\}, \\
& F(T, V, N) \rightarrow \text { min subject to given } T, V \text { and }\left\{B_{j}\right\}, \\
& G(T, P, N) \rightarrow \text { min subject to given } T, P \text { and }\left\{B_{j}\right\} .
\end{aligned}
$$

For the free entropies the equilibrium should be the maximizer: in addition to (3.1)

$$
\begin{aligned}
& \Phi\left(\frac{1}{T}, V, N\right) \rightarrow \max \text { subject to given } \frac{1}{T}, V \text { and }\left\{B_{j}\right\}, \\
& \Xi\left(\frac{1}{T}, \frac{P}{T}, N\right) \rightarrow \max \text { subject to given } \frac{1}{T}, \frac{P}{T} \text { and }\left\{B_{j}\right\} .
\end{aligned}
$$

In extensive thermodynamics, $V, U, S$, and $N$ are the extensive variables, that is they are directly proportional to the system volume if we just join several copies of the system with the proportional increase of the volume. Therefore, $U(S, V, N)$ is the homogeneous function of the first order, and the equation for $\mathrm{d} U$ can be easily integrated (this is the Euler theorem):

$$
U=T S-P V+\sum_{i} \mu_{i} N_{i}
$$

In this case,

$$
\begin{gathered}
H=U+P V=T S+\sum_{i} \mu_{i} N_{i}, \\
F=U-T S=-P V+\sum_{i} \mu_{i} N_{i}, \\
G=H-T S=\sum_{i} \mu_{i} N_{i}, \\
\Omega=U-T S-\sum_{i} \mu_{i} N_{i}=-P V,
\end{gathered}
$$

Free energies have a very important physical and technical sense. They measure the available work under given conditions.

Free entropies coincide (up to some constant additions) with the entropies of the minimal isolated system, which includes the system under consideration. This statement was analyzed in detail in [42] but is still not very well known. Therefore, let us prove it. 
Physically, when we consider a system under isothermal condition, this means that the system is in contact with a large thermal bath. The state of the thermal bath is characterized by two variables, $U_{\mathrm{T}}$ and $V_{\mathrm{T}}$. The entropy of a thermal bath is $S_{\mathrm{T}}\left(U_{\mathrm{T}}, V_{\mathrm{T}}\right)$. The total entropy of the isolated system "a system + the thermal bath" is

$$
\widetilde{S}\left(U, V, N, U_{\mathrm{T}}, V_{\mathrm{T}}\right)=S(U, V, N)+S_{\mathrm{T}}\left(U_{\mathrm{T}}, V_{\mathrm{T}}\right) .
$$

The equilibrium is the maximizer of the total entropy $\widetilde{S}$ for given total energy $\widetilde{U}=U+U_{\mathrm{T}}$, values of volumes $V, V_{\mathrm{T}}$ and linear conservation laws $\left\{B_{j}\right\}$. In particular,

$$
\begin{aligned}
& \frac{\partial\left[S(U, V, N)+S_{\mathrm{T}}\left(\widetilde{U}-U, V_{\mathrm{T}}\right)\right]}{\partial U}=0, \\
& \frac{\partial S(U, V, N)}{\partial U}=\left.\frac{\partial S_{\mathrm{T}}\left(U_{\mathrm{T}}, V_{\mathrm{T}}\right)}{\partial U}\right|_{U_{\mathrm{T}}=\widetilde{U}-U} .
\end{aligned}
$$

This means that the temperatures of the thermal bath and the system are equal, $T=T_{\mathrm{T}}$.

Let us take the conditional maximum function (under conditions $U+U_{\mathrm{T}}=\widetilde{U}, T=T_{\mathrm{T}}$ :

$$
\begin{aligned}
S_{\Phi}\left(\widetilde{U}, V, N, V_{\mathrm{T}}\right) & =S(U, V, N)+S_{\mathrm{T}}\left(\widetilde{U}-U, V_{\mathrm{T}}\right) \\
& =S(U, V, N)+V_{\mathrm{T}} S_{\mathrm{T}}\left(\frac{\widetilde{U}-U}{V_{\mathrm{T}}}, 1\right) \\
& =S(U, V, N)+V_{\mathrm{T}} S_{\mathrm{T}}\left(\frac{\widetilde{U}}{V_{\mathrm{T}}}, 1\right)-\frac{U}{T} \\
& =\Phi+V_{\mathrm{T}} S_{\mathrm{T}}\left(\frac{\widetilde{U}}{V_{\mathrm{T}}}, 1\right)+O\left(V_{\mathrm{T}}^{-1}\right)
\end{aligned}
$$

This function differs from the free entropy $\Phi$ by a constant $S_{\mathrm{T}}\left(\widetilde{U}, V_{\mathrm{T}}\right)$ and an infinitesimal $O\left(V_{\mathrm{T}}^{-1}\right)$, which goes to zero when the bath increases.

Therefore, the free entropy $\Psi$ (the Massieu function) is equal to the entropy of the minimal isolated system, which includes the system under consideration and the large thermal bath (up to a large constant and an infinitesimal additions).

For the Planck function $\Xi$ we have to consider a system under a constant pressure in the contact with the same large thermal bath. The only difference is that instead of the internal energy of our system we have to take the enthalpy. The enthalpy is the energy of the system plus the device, which keeps the pressure constant (potential energy of a heavy piston of the given weight). So, the total energy is the energy of the minimal isolated system $\widetilde{U}=H+U_{\mathrm{T}}$ and everything else is the same as for $\Phi$ : the free entropy is the entropy of the minimal isolated system, which includes the system under consideration, under condition of the partial equilibrium with auxiliary systems and up to a constant summand. 
For perfect systems, by definition,

$$
\begin{aligned}
& U=\sum_{i} N_{i} u_{i}(T), \\
& P V=R T \sum_{i} N_{i},
\end{aligned}
$$

where $u_{i}(T)$ is the energy of one mole of $A_{i}$ at the temperature $T$.

Under this assumption, the entropy $S$ is defined up to an arbitrary uniform function of first order $S_{0}(N)$ :

$$
S=R S_{0}(N)+\sum_{i} N_{i}\left[-R \ln c_{i}+\int_{T_{0}}^{T} \frac{1}{\tau} \frac{\mathrm{d} u_{i}(\tau)}{\mathrm{d} \tau} \mathrm{d} \tau\right] .
$$

If we assume that $S_{0}(N)$ is a linear function,

$$
S_{0}(N)=\sum_{i} \delta_{i} N_{i}
$$

then

$$
S=\sum_{i} N_{i}\left[-R\left(\ln c_{i}-\delta_{i}\right)+\int_{T_{0}}^{T} \frac{1}{\tau} \frac{\mathrm{d} u_{i}(\tau)}{\mathrm{d} \tau} \mathrm{d} \tau\right],
$$

where $c_{i}=N_{i} / V$ is the concentration, $T_{0}>0$ is a reference temperature (we assume that on the interval $\left[T_{0}, T\right]$ the system is perfect (3.7)).

It is necessary to stress that linearity of $S_{0}(N)$ does not follow from the assumption (3.7) and is an additional hypothesis. For a general perfect system (3.7) we can state that $S_{0}(N)$ is a uniform function of the first order only.

Formulas (3.7), (3.9) allow us to express the free energy in the proper variables, $F(T, V, N)$ :

$$
\begin{aligned}
F(T, V, N) & =U-T S \\
& =\sum_{i} N_{i} u_{i}(T)+R T \sum_{i} N_{i}\left[\ln \left(\frac{N_{i}}{V}\right)-\delta_{i}-\int_{T_{0}}^{T} \frac{1}{R \tau} \frac{\mathrm{d} u_{i}(\tau)}{\mathrm{d} \tau} \mathrm{d} \tau\right] .
\end{aligned}
$$

From this formula for $F(T, V, N)$, all other thermodynamic functions can be expressed locally:

$$
S=\frac{\partial F}{\partial T}, U=F-T \frac{\partial F}{\partial T}, P=-\frac{\partial F}{\partial V}, \mu_{i}=\frac{\partial F}{\partial N_{i}}, \ldots
$$

Generalizations of the free energy for non-perfect systems are often produced by transformations of (3.10). The first generalization describes a system of small admixtures to a general system. Let the "background" system have the extensive state variables $M$. Interaction of small admixtures is negligible and the formula $P V=R T \sum N_{i}$ describes the osmotic pressure of the admixtures.. Then we can write, analogously to (3.10):

$$
\begin{aligned}
F(T, V, N, M)= & F_{0}(T, V, M)+\sum_{i} N_{i} u_{i}\left(T, \frac{M}{V}\right) \\
& +R T \sum_{i} N_{i}\left[\ln \left(\frac{N_{i}}{V}\right)-\delta_{i}\left(\frac{M}{V}\right)-\int_{T_{0}}^{T} \frac{1}{R \tau} \frac{\mathrm{d} u_{i}\left(\tau, \frac{M}{V}\right)}{\mathrm{d} \tau} \mathrm{d} \tau\right] .
\end{aligned}
$$


Here, the energies $u_{i}$ and parameters $\delta_{i}$ are functions of densities $M / V$. For each given value of these densities, this formula coincides with (3.10).

The first model of non-perfect gases is the van der Waals gas. To write the free energy for this type of gas (or gas of admixtures) we have to take into account two effects: the excluded volume per mole of particles $A_{i}, v_{i}$, and the energy of attraction for particles $A_{i}, A_{j}$ with the energy density

$$
\epsilon_{i j}=-a_{i j} c_{i} c_{j}
$$

(minus because this is attraction).

For the free energy these effects give:

$$
\begin{aligned}
F(T, V, N)= & \sum_{i} N_{i} u_{i}(T)-V \sum_{i j} a_{i j} \frac{N_{i}}{V} \frac{N_{j}}{V} \\
& +R T \sum_{i} N_{i}\left[\ln \left(\frac{N_{i}}{V-\sum_{i} v_{i} N_{i}}\right)-\delta_{i}-\int_{T_{0}}^{T} \frac{1}{R \tau} \frac{\mathrm{d} u_{i}(\tau)}{\mathrm{d} \tau} \mathrm{d} \tau\right] .
\end{aligned}
$$

For adsorbed particles on a surface, the model of an ideal adsorbed layer implies a lattice of places, and a multicomponent gas with components $A_{0}=Z, A_{1}, \ldots, A_{n}$ where $Z$ ia a free place and $A_{i}$ are adsorbed particles on their places (each adsorbed particle occupies a place). There is a conservation law:

$$
\sum_{i=0}^{n} c_{i}=\theta=\mathrm{const},
$$

therefore, $c_{0}=\theta-\sum_{i=1}^{n} c_{i}$ and the free energy has the form of the energy of the Fermi-gas:

$$
\begin{aligned}
F(T, \sigma, N)= & F_{0}(T) \sigma+\sum_{i=1}^{n} N_{i} u_{i}(T) \\
& +R T \sum_{i=1}^{n} N_{i}\left[\ln \left(\frac{N_{i}}{\sigma}\right)-\delta_{i}-\int_{T_{0}}^{T} \frac{1}{R \tau} \frac{\mathrm{d} u_{i}(\tau)}{\mathrm{d} \tau} \mathrm{d} \tau\right] \\
& +\left(\sigma \theta-\sum_{i=1}^{n} N_{i}\right)\left(\ln \left(\theta-\sum_{i=1}^{n} \frac{N_{i}}{\sigma}\right)-\delta_{0}\right)
\end{aligned}
$$

where $\sigma$ is the surface area, $F_{0}(T) \sigma$ is the free energy of empty surface.

For the systems, distributed in space, the density of free energy may be expressed through the concentrations, for example, for the perfect system (3.10)

$$
\begin{aligned}
f(T, c) & =\frac{F(T, V, c V)}{V} \\
& =\sum_{i} c_{i} u_{i}(T)+R T \sum_{i} c_{i}\left[\ln c_{i}-\delta_{i}-\int_{T_{0}}^{T} \frac{1}{R \tau} \frac{\mathrm{d} u_{i}(\tau)}{\mathrm{d} \tau} \mathrm{d} \tau\right] .
\end{aligned}
$$


Therefore, for non-uniform system

$$
F=\int_{V} f(T(x), c(x)) \mathrm{d} x .
$$

This formula is applicable if the space gradients are not too sharp. If $f(T, c)$ is a convex function of $c$ then the minimizer of $F$ (3.15) under given $T, V$ and $N=\int_{V} c(x \mathrm{~d} x$ (i.e. the equilibrium) is a uniform distribution and we should not expect spontaneous appearance of singularities on the way to the equilibrium. If $f(T, c)$ is not a convex function of $c$ then the minimizer of $F$ may be nonuniform, non-smooth and non-unique: phase transitions are possible. In that case, the simple integral of the density $f$ should be regularized by addition terms. Now, the standard approach gives the Ginzburg-Landau free energy:

$$
\begin{aligned}
& F=\int_{V} \psi(T(x), c(x), \nabla c(x)) \mathrm{d} x \\
& \psi(T(x), c(x), \nabla c(x))=f(T(x), c(x))+\frac{1}{2} \sum_{i} \lambda_{i}\left(\nabla c_{i}\right)^{2}
\end{aligned}
$$

More general dependencies of $\nabla c$ are also under consideration [58]. The chemical potentials $\mu_{i}$ for the free energy (3.16) are defined as variational derivatives of this functional,

$$
\mu_{i}=\frac{\delta F}{\delta c_{i}}=\frac{\partial f(T, c)}{\partial c_{i}}-\lambda_{i} \Delta c_{i}
$$

Special analysis of the most general form of diffusion equations which provide the proper decrease of the Ginzburg-Landau free energy (3.16) was provided by Gurtin [58]. He introduced general nonlinear mobility matrices.

The kinetic laws should satisfy the thermodynamic restrictions. That is, the dissipation should be positive. There are two physical forms of this law: (i) the available work should decrease and (ii) the entropy of the minimal isolated system, which includes the system under consideration, should increase. These two equivalent formulations correspond to two series of thermodynamic potentials: energetic or entropic series. There are several approaches to a general formalisms, which pretend to describe all systems that satisfy these monotonicity conditions [56, 87]. Such approaches form the special discipline, nonequilibrium thermodynamics [20, 59, 72] or beyond equilibrium thermodynamics [85].

Our goal is different: we construct a method for assembling of a complex transport process from a mechanism combined by simple elementary processes. This approach for physics should satisfy the basic thermodynamic requirements.

\subsubsection{Markovian Microkinetics and Generalized Mass Action Law}

To satisfy the thermodynamic restrictions the kinetic law of the elementary reactions should have a special form, and the reaction rate constants for different elementary reactions should be harmonized. 
Let us start from a reaction mechanism given by the stoichiometric equations (2.1):

$$
\sum_{i} \alpha_{r i} A_{i} \rightarrow \sum_{i} \beta_{r i} A_{i}
$$

where $r$ is a reaction number, $\alpha_{r i}$ and $\beta_{r i}$ are nonnegative numbers, the stoichiometric coefficients.

In this Subsection, we return to some ideas of Michaelis and Menten [80] and to the Stueckelberg analysis of the Boltzmann equation [97] and represent the general kinetic law for elementary processes. Detailed analysis was provided recently in [54].

This law could be proved under the following assumptions:

1. The elementary processes go through intermediate states (complexes or compounds) 1.44 (Fig. 5):

$$
\sum_{i} \alpha_{r i} A_{i} \rightleftharpoons B_{r}^{-} \rightarrow B_{r}^{+} \rightleftharpoons \sum_{i} \beta_{r i} A_{i}
$$

where $\rho$ is the elementary process number;

2. The amount of each compound $B_{\rho}$ is small enough to apply the perfect free energy formula (3.11) for them;

3. The equilibrium between each compound and the corresponding linear combinations of reagents is fast enough to apply the quasiequilibrium approximation [54];

4. The transitions between compounds could be described by a continuous time Markov chain (the Master equation or the monomolecular kinetics).

The first three items of these assumptions correspond exactly to the celebrated MichaelisMenten work [80]. Later, Briggs and Haldane [7] abandoned the assumption of fast equilibria and produced the so-called Michaelis and Menten kinetics approximation. Original approach of Michaelis and Menten (fast equilibria with intermediate compounds + small amounts of compounds) was discovered again by Stueckelberg [97] almost 30 years later. It was applied not to the kinetics of catalytic reactions but to the collision in the gas kinetics, as an alternative background of the Boltzmann equation. Gorban and Shahzad [54] provided the detailed analysis of this approach to the general kinetic equation.

Let the concentration of the intermediate compound $B_{\rho}$ (1.44) be $\varsigma_{\rho}$. The free energy (3.11) for the small admixture of compounds $B_{\rho}$ to the components $A_{i}$ may be represented in the form

$$
F=V f(c, T)+V R T \sum_{\rho=1}^{q} \varsigma_{\rho}\left(\ln \left(\frac{\varsigma_{\rho}}{\varsigma_{\rho}^{*}(c, T)}\right)-1\right)
$$

where $\varsigma_{\rho}$ is the standard equilibrium concentrations of $B_{\rho}$.

We assume that the standard equilibrium concentrations $\varsigma_{\rho}^{*}(c, T)$ as well as the current concentrations $\varsigma_{\rho}$ are much smaller than the concentrations of $A_{i}$. 
To formulate the results of Michaelis-Menten-Stueckelberg-Gorban-Shahzad (MMSGS kinetics) we have to introduce the basic notions in more detail. First of all, some of the formal linear combinations

$$
\left(\alpha_{r}, A\right)=\sum_{i} \alpha_{r i} A_{i},\left(\beta_{r}, A\right)=\sum_{i} \beta_{r i} A_{i}
$$

may coincide. The same combination may be, simultaneously, the input combination of several reactions and the output combination of several other reactions.

We assume that a fast intermediate compound $B \ldots$ corresponds not to a reaction but to a formal complex of the form $\left(\alpha_{r}, A\right)$ or $\left(\beta_{r}, A\right)$ and this compound is the same for all reactions which include this complex.

Let us call the formal linear combinations of the form $\left(\alpha_{r}, A\right)$ or $\left(\beta_{r}, A\right)$ the complexes and enumerate them: $\Theta_{1}, \Theta_{2}, \ldots, \Theta_{q}$. For each complex $\Theta_{j}$, the corresponding vector of coefficients $\left(\alpha_{r}\right.$ or $\left.\beta_{r}\right)$ is $y_{j}: \Theta_{j}=\left(y_{j}, A\right)$.

The reaction mechanism (3.18) may be represented in the form $\Theta_{j} \rightarrow \Theta_{s}$ for some pairs $(j, s)$.

The additional component, the fast compound $B_{j}$, corresponds to each complex $\Theta_{j}$ and the reaction mechanism $\Theta_{j} \rightarrow \Theta_{s}$ (for some pairs $(j, s)$ ) (3.18) is extended to

$$
\Theta_{j} \rightleftharpoons B_{j} \rightarrow B_{s} \rightleftharpoons \Theta_{s} \text { for some pairs }(j, s) \text {. }
$$

The fast equilibrium $\Theta_{j} \rightleftharpoons B_{j}$ gives

$$
\vartheta_{j}=\sum_{i} y_{j i} \frac{\mu_{i}(c, T)}{R T},
$$

or

$$
\varsigma_{j}=\varsigma_{j}^{*}(c, T) \exp \left(\frac{\sum_{i} y_{j i} \mu_{i}(c, T)}{R T}\right)
$$

where

$$
\mu_{i}=\frac{\partial f(c, T)}{\partial c_{i}}
$$

is the chemical potential of $A_{i}$ and

$$
\vartheta_{j}=\ln \left(\frac{\varsigma_{j}}{\varsigma_{j}^{*}}\right)
$$

$\left(R T \vartheta_{j}=\frac{1}{V} \frac{\partial F}{\partial \varsigma_{j}}\right.$ is the chemical potential of $\left.B_{j}\right)$.

For the systems with fixed volume, the stoichiometric conservation laws of the monomolecular system of reaction between compounds are sums of the concentrations of $B_{j}$ which belong to various connected components of the reaction graph. Under the hypothesis of weak reversibility there is no other conservation law.

Let the graph of reactions $B_{j} \rightarrow B_{l}$ have $d$ connected components $C_{s}$ and let $V_{s}$ be the set of indexes of those $B_{j}$ which belong to $C_{s}: B_{j} \in C_{s}$ if and only if $j \in V_{s}$. For each $C_{s}$ there exists a stoichiometric conservation law

$$
\beta_{s}=\sum_{j \in V_{s}} \varsigma_{j} .
$$


For any set of positive values of $\beta_{s}(s=1, \ldots, d)$ and given $c, T$ there exists a unique conditional maximizer $\varsigma_{j}^{\text {eq }}$ of the free energy (3.20): for the compound $B_{j}$ from the $s$ th connected component $\left(j \in V_{s}\right)$ this equilibrium concentration is

$$
\varsigma_{j}^{\mathrm{eq}}=\beta_{s} \frac{\varsigma_{j}^{*}(c, T)}{\sum_{l \in V_{s}} \varsigma_{j}^{*}(c, T)}
$$

Inversely, positive values of concentrations $\varsigma_{j}$ are the equilibrium concentrations (3.25) for some values of $\beta_{s}$ if and only if for any $s=1, \ldots, d$

$$
\vartheta_{j}=\vartheta_{l} \text { if } j, l \in V_{s}
$$

$\left(\vartheta_{j}=\ln \left(\varsigma_{j} / \varsigma_{j}^{*}\right)\right)$. This system of equations together with the equilibrium conditions (3.23) constitute the equilibrium of the systems. All the equilibria form a linear subspace in the space with coordinates $\mu_{i} / R T(i=1, \ldots, n)$ and $\vartheta_{j}(j=1, \ldots, q)$.

Our expression for the free energy does not assume anything special about the free energy of the mixture of $A_{i}$. For the compounds $B_{i}$, we assume that this is a very small addition to the mixture of $A_{i}$, neglect all quadratic terms in concentrations of $B_{i}$ and use the free energy of the perfect systems for this small admixture.

The Master Equation for the concentration of $B_{j}$ gives:

$$
\frac{\mathrm{d} \varsigma_{j}}{\mathrm{~d} t}=\sum_{l, l \neq j}\left(\kappa_{j l} \varsigma_{l}-\kappa_{l j} \varsigma_{j}\right) .
$$

This kinetic equation should respect thermodynamics. For the Master equation this means the equilibrium condition

$$
\sum_{l, l \neq j} \kappa_{j l} \varsigma_{l}^{*}=\sum_{l, l \neq j} \kappa_{l j} \varsigma_{j}^{*} .
$$

Under this condition, the Master Equation (3.27) has the equivalent form:

$$
\frac{\mathrm{d} \varsigma_{j}}{\mathrm{~d} t}=\sum_{l, l \neq j} \kappa_{j l} \varsigma_{l}^{*}\left(\frac{\varsigma_{l}}{\varsigma_{l}^{*}}-\frac{\varsigma_{j}}{\varsigma_{j}^{*}}\right) .
$$

In this form, it is obvious that $\varsigma_{j}^{*}$ is equilibrium for the kinetic equation.

All these expressions for concentrations of compounds and the Markov reaction rates result in the following kinetic law:

1. The reaction rate of the reaction $\Theta_{j} \rightarrow \Theta_{s}$ is

$$
w_{s j}=\phi_{s j} \exp \left(\frac{\sum_{i} y_{j i} \mu_{i}(c, T)}{R T}\right),
$$

where the quantity $\phi_{s j} \geq 0$ is a kinetic factor, in the Markov model it corresponds to $\kappa_{s j} \varsigma_{j}^{*}$; 
2. The kinetic factors $\phi_{s j}$ satisfy the complex balance condition:

$$
\sum_{s} \phi_{j s}=\sum_{s} \phi_{s j} \text { for all } j
$$

in the Markov model this identity corresponds to the equilibrium condition (3.28).

This is the macroscopic MMSGS kinetics.

For this kinetics, the free energy decreases in time. To demonstrate this dissipation inequality, let us formulate the macroscopic MMSGS kinetic equations in the original notations for the reaction mechanism (3.18). Formula (3.30) for the reaction rate gives

$$
w_{r}=\phi_{r} \exp \left(\frac{\sum_{i} \alpha_{r i} \mu_{i}(c, T)}{R T}\right)
$$

The kinetic equation under the isochoric conditions (the constant volume) are

$$
\frac{\mathrm{d} c}{\mathrm{~d} t}=\sum_{r} \gamma_{r} w_{r}
$$

where the stoichiometric vector $\gamma_{r}=\beta_{r}-\alpha_{r}$. According to this equation, the dissipation rate is

$$
\frac{\mathrm{d} F}{\mathrm{~d} t}=V \sum_{r}\left(\mu, \gamma_{r}\right) w_{r}=V \sum_{r} \phi_{r}\left(\mu,\left(\beta_{r}-\alpha_{r}\right)\right) \exp \left(\frac{\left(\alpha_{r}, \mu\right)}{R T}\right),
$$

where $\mu_{i}=\partial F / \partial N_{i}$ is the chemical potential.

Let us introduce an auxiliary function like we did it for MAL (2.17):

$$
\theta(\lambda)=\sum_{r} \phi_{r} \exp \left[\frac{\lambda\left(\alpha_{r}, \mu\right)+(1-\lambda)\left(\beta_{r}, \mu\right)}{R T}\right] .
$$

This function is convenient because

$$
\frac{\mathrm{d} \theta(\lambda)}{\mathrm{d} \lambda}=-\sum_{r} \phi_{r}\left(\mu,\left(\beta_{r}-\alpha_{r}\right)\right) \exp \left[\frac{\lambda\left(\alpha_{r}, \mu\right)+(1-\lambda)\left(\beta_{r}, \mu\right)}{R T}\right] .
$$

Therefore, for the dissipation rate we get

$$
\frac{\mathrm{d} F}{\mathrm{~d} t}=-V \theta^{\prime}(1)
$$

The function $\theta(\lambda)$ is a sum of exponents. It is convex $\left(\theta^{\prime \prime}(\lambda) \geq 0\right)$. Therefore, if $\theta(0)=\theta(1)$ then $\theta^{\prime}(1) \geq 0$. This means that the identity

$$
\theta(0) \equiv \theta(1)
$$


is a sufficient condition for the dissipation inequality

$$
\frac{\mathrm{d} F}{\mathrm{~d} t} \leq 0
$$

Some of vectors $\alpha_{r}, \beta_{r}$ may coincide. Let there be $q$ different vectors among them. We denote them $y_{1}, \ldots, y_{q}$. For each $y_{i}$ we define $R_{i}^{+}=\left\{r \mid \alpha_{r}=y_{i}\right\}, R_{i}^{-}=\left\{r \mid \beta_{r}=y_{i}\right\}$. The sufficient condition for the identity (3.38) is

$$
\sum_{r \in R_{i}^{+}} \phi_{r}=\sum_{r \in R_{i}^{-}} \phi_{r} \text { for all } i .
$$

This condition is also necessary if we can vary $\phi_{r}$ and $\mu_{i}$ independently and the Jacobian $\left|\partial \mu_{i} / \partial c_{j}\right|$ has the full rank. This condition is the complex balance condition.

Of course, the important particular case of the complex balance conditions is the detailed balance condition:

$$
\phi_{r}^{+}=\phi_{r}^{-},
$$

where $\phi_{r}^{+}, \phi_{r}^{-}$are the kinetic factors of the mutually reverse reactions: $\phi_{r}^{+}$for $\left(\alpha_{r}, A\right) \rightarrow\left(\beta_{r}, A\right)$ and $\phi_{r}^{-}$for $\left(\beta_{r}, A\right) \rightarrow\left(\alpha_{r}, A\right)$.

\subsection{From Cell-Jump Models to Continuous Diffusion Equations for Generalized Mass Action Law}

Let us start again from the stoichiometric mechanism of the diffusion:

$$
\sum_{i} \alpha_{r i}^{\mathrm{I}} A_{i}^{\mathrm{I}}+\sum_{i} \alpha_{r i}^{\mathrm{II}} A_{i}^{\mathrm{II}} \rightarrow \sum_{i} \beta_{r i}^{\mathrm{I}} A_{i}^{\mathrm{I}}+\sum_{i} \beta_{r i}^{\mathrm{II}} A_{i}^{\mathrm{II}}
$$

All the elementary processes have different numbers $r$ and the space-inverted and reverse processes are represented separately. Let us consider all pairs of vectors, $\left(\alpha_{r}^{\mathrm{I}}, \alpha_{r}^{\mathrm{II}}\right)$ and $\left(\beta_{r}^{\mathrm{I}}, \beta_{r}^{\mathrm{II}}\right)$ and numerate all the different pairs: $y_{1}, y_{2}, \ldots$, where $y_{q}=\left(y_{q}^{\mathrm{I}}, y_{q}^{\mathrm{II}}\right)$. For each $y_{q}$ there are two sets of reactions, $R_{q}^{+}, R_{q}^{-}$:

$$
R_{q}^{+}=\left\{r \mid\left(\alpha_{r}^{\mathrm{I}}, \alpha_{r}^{\mathrm{II}}\right)=y_{q}\right\}, R_{q}^{-}=\left\{r \mid\left(\beta_{r}^{\mathrm{I}}, \beta_{r}^{\mathrm{II}}\right)=y_{q}\right\} .
$$

The reaction rate for the elementary process (3.41) is (3.32):

$$
w_{r}=\phi_{r} \exp \left(\frac{\left(\alpha_{r}^{\mathrm{I}}, \mu\left(c^{\mathrm{I}}, T\right)\right)+\left(\alpha_{r}^{\mathrm{II}}, \mu\left(c^{\mathrm{II}}, T\right)\right)}{R T}\right),
$$

The kinetic factors are functions of $c^{\mathrm{I}}, c^{\mathrm{II}}$ and $T$. They should satisfy the identity of complex balance (3.39). 
The cell-jump model gives us

$$
\begin{aligned}
J & =-\sum_{r} \gamma_{r} w_{r}(c(x), c(x+l)) \\
& =\sum_{q}\left(\sum_{r \in R_{q}^{+}} \alpha_{r}^{\mathrm{I}} w_{r}(c(x), c(x+l))-\sum_{r \in R_{q}^{-}} \beta_{r}^{\mathrm{I}} w_{r}(c(x), c(x+l))\right) \\
& =\sum_{q} y_{q}^{\mathrm{I}}\left(\sum_{r \in R_{q}^{+}} w_{r}(c(x), c(x+l))-\sum_{r \in R_{q}^{-}} w_{r}(c(x), c(x+l))\right)
\end{aligned}
$$

The zero order term in $l$ is

$$
\sum_{q} y_{q}^{\mathrm{I}} \exp \left(\frac{\left(y_{q}^{\mathrm{I}}+y_{q}^{\mathrm{II}}, \mu\right)}{R T}\right)\left(\sum_{r \in R_{q}^{+}} \phi_{r}-\sum_{r \in R_{q}^{-}} \phi_{r}\right)=0 .
$$

It vanishes because of the complex balance condition.

The first order term gives

$$
\begin{aligned}
J=l \sum_{q} y_{q}^{\mathrm{I}} \exp \left(\frac{\left(y_{q}^{\mathrm{I}}+y_{q}^{\mathrm{II}}, \mu\right)}{R T}\right) & \left(\sum_{r \in R_{q}^{+}} \phi_{r}\left(\alpha_{r}^{\mathrm{II}}, \nabla\left(\frac{\mu}{R T}\right)\right)\right. \\
& \left.-\sum_{r \in R_{q}^{-}} \phi_{r} \sum_{j}\left(\alpha_{r}^{\mathrm{II}}, \nabla\left(\frac{\mu}{R T}\right)\right)\right) .
\end{aligned}
$$

Each term in this sum consists of the positive scalar pre-factor

$$
l \exp \left(\frac{\left(y_{q}^{\mathrm{I}}+y_{q}^{\mathrm{II}}, \mu\right)}{R T}\right)
$$

and the matrix

$$
y_{q i}^{\mathrm{I}}\left(\sum_{r \in R_{q}^{+}} \phi_{r} \alpha_{r j}^{\mathrm{II}}-\sum_{r \in R_{q}^{-}} \phi_{r} \alpha_{r j}^{\mathrm{II}}\right)
$$

multiplied by

$$
\nabla\left(\frac{\mu_{j}}{R T}\right)
$$

This structure of the formula for the flux is very similar to (2.55). Let us follow the same logic as in Subsection 2.5. to find the more convenient form of the expression for the flux (3.44). First of all, let us group all terms with the same pre-factor. 
For the set of all vectors $y_{q}^{\mathrm{I}}+y_{q}^{\mathrm{II}}$ we use notation $Z$. For each $z \in Z$

$$
Y_{z}=\left\{y_{q} \mid y_{q}^{\mathrm{I}}+y_{q}^{\mathrm{II}}=z\right\} .
$$

Analogously to (2.56) we get

$$
\begin{aligned}
& J=l \sum_{z \in Z} l \exp \left(\frac{\left(y_{q}^{\mathrm{I}}+y_{q}^{\mathrm{II}}, \mu\right)}{R T}\right) J^{z}, \\
& J^{z}=\sum_{y_{q} \in Y_{z}} y_{q}^{\mathrm{I}} \sum_{j}\left(\sum_{r \in R_{q}^{+}} \phi_{r} \alpha_{r j}^{\mathrm{II}}-\sum_{r \in R_{q}^{-}} \phi_{r} \alpha_{r j}^{\mathrm{II}}\right) \nabla\left(\frac{\mu_{j}}{R T}\right) .
\end{aligned}
$$

Let us analyze $J^{z}$ for given $z$.

First of all,

$$
\sum_{y_{q} \in Y_{z}} \sum_{j}\left(\sum_{r \in R_{q}^{+}} \phi_{r} \alpha_{r j}^{\mathrm{II}}-\sum_{r \in R_{q}^{-}} \phi_{r} \alpha_{r j}^{\mathrm{II}}\right) \nabla\left(\frac{\mu_{j}}{R T}\right)=0
$$

because each $\phi_{r}$ from this sum enters it twice, with the opposite signs, one time as an element of the sum over $R_{q}^{+}$for some $y_{q} \in Y_{z}$ (with + ) and the second time (with -) as an element of the sum over $R_{s}^{-}$for another $y_{s} \in Y_{z}$ with the same sum

$$
y_{s}^{\mathrm{I}}+y_{s}^{\mathrm{II}}=y_{q}^{\mathrm{I}}+y_{q}^{\mathrm{II}}=z .
$$

(Let us recall that

$$
\alpha_{s}^{\mathrm{I}}+\alpha_{s}^{\mathrm{II}}=\beta_{s}^{\mathrm{I}}+\beta_{s}^{\mathrm{II}}
$$

for all $s$ and, hence, if $r \in R_{q}^{+}$and $\alpha_{r}^{\mathrm{I}}+\alpha_{r}^{\mathrm{II}}=z$ then $\beta_{s}^{\mathrm{I}}+\beta_{s}^{\mathrm{II}}=z$ and $\left(\beta_{s}^{\mathrm{I}}, \beta_{s}^{\mathrm{II}}\right) \in Y_{z}$.)

Let us mention that $y_{q}^{\mathrm{I}}=z-y_{q}^{\mathrm{II}}$. Therefore,

$$
J^{z}=-\sum_{y_{q} \in Y_{z}} y_{q}^{\mathrm{II}} \sum_{j}\left(\sum_{r \in R_{q}^{+}} \phi_{r} \alpha_{r j}^{\mathrm{II}}-\sum_{r \in R_{q}^{-}} \phi_{r} \alpha_{r j}^{\mathrm{II}}\right) \nabla\left(\frac{\mu_{j}}{R T}\right) .
$$

In this formula, all the stoichiometric vectors are for the same cell, for the second one.

Let us demonstrate similarity of the expression (3.46) to some formulas from the theory of Markov chains. Let us follow Subsection 2.5. and numerate the states of the auxiliary Markov chain by vectors $y_{q} \in Y_{z}$. Elementary processes correspond to transitions between states. Each nonzero kinetic factor $\phi_{r}$ corresponds to two vectors $y_{q}, y_{s} \in Y_{z}: r \in R_{q}^{+}$and $r \in R_{s}^{-}$.

Let us substitute the index $r$ by two indexes $q, s$ and use notation $\phi_{s q}$ for transitions $y_{q} \rightarrow y_{s}$ (i.e. for the case $r \in R_{q}^{+}$and $r \in R_{s}^{-}$).

The complex balance condition (3.39) reads:

$$
\sum_{q} \phi_{s q}=\sum_{q} \phi_{q s}
$$


This is precisely the steady state condition for the Markov chain.

Inequality (2.64) holds for the kinetic factors:

$$
\sum_{s q} \pi_{q}\left(\phi_{q s} \pi_{s}-\phi_{s q} \pi_{q}\right)=\sum_{s q} \pi_{q} \phi_{q s}\left(\pi_{s}-\pi_{q}\right) \leq 0
$$

for any set of numbers $\pi_{q}$.

For the proof of the dissipation inequality it is convenient to rewrite $J^{z}$ in these notations:

$$
J^{z}=\sum_{y_{q} \in Y_{z}} y_{q}^{\mathrm{II}} \sum_{j} \sum_{s}\left(\phi_{q s} y_{s j}^{\mathrm{II}}-\phi_{s q} y_{q j}^{\mathrm{II}}\right) \nabla\left(\frac{\mu_{j}}{R T}\right) .
$$

Free energy in a domain $V$ is

$$
\mathbf{F}=\int_{V} f(c, T) \mathrm{d} x .
$$

Let us estimate $\dot{\mathbf{F}}$ in a bounded domain $V$ with smooth boundary and without fluxes through boundary for isothermal conditions.

$$
\begin{aligned}
\frac{\mathrm{d} \mathbf{F}}{\mathrm{d} t} & =R T \int_{V} \sum_{j}\left(\nabla_{x}\left(\frac{\mu_{j}}{R T}\right), J_{j}\right) \mathrm{d} x \\
& =l \sum_{z \in Z} \int_{V} \exp \left(\frac{\left(y_{q}^{\mathrm{I}}+y_{q}^{\mathrm{II}}, \mu\right)}{R T}\right) \sum_{j}\left(\nabla_{x}\left(\frac{\mu_{j}}{R T}\right), J_{j}^{z}\right) \mathrm{d} x .
\end{aligned}
$$

Due to the representation of $J^{z}(3.49)$,

$$
\sum_{j}\left(\nabla_{x}\left(\frac{\mu_{j}}{R T}\right), J_{j}^{z}\right)=\sum_{q s}\left(\pi_{q},\left(\phi_{q s} \pi_{s}-\phi_{s q} \pi_{q}\right)\right) \leq 0,
$$

where $\pi_{q}$ is a space vector: $\pi_{q}=\sum_{i} y_{q i}^{\mathrm{II}} \nabla_{x}\left(\frac{\mu_{j}}{R T}\right)$ and for $y_{q}, y_{s}$

$$
y_{q, s}^{\mathrm{I}}+y_{q, s}^{\mathrm{II}}=z
$$

This expression has exactly the form (3.48) for each space coordinate. Finally,

$$
\sum_{j}\left(\nabla_{x}\left(\frac{\mu_{j}}{R T}\right), J_{j}^{z}\right) \leq 0
$$

and it is zero if all $\pi_{q}=\sum_{i} y_{q i}^{\mathrm{II}} \nabla \ln c_{i}$ coincide. (The reverse statement is correct if the auxiliary Markov chain with the transition coefficients $\phi_{q s}$ is ergodic for given $z$.)

Therefore,

$$
\dot{\mathbf{F}} \leq 0
$$

for the generalized MAL (3.30) with the complex balance conditions (3.31). 


\subsection{Detailed Balance for the Generalized Mass Action Law and the Dissipation Inequality}

\subsubsection{Isothermal conditions}

Systems with detailed balance form an important subclass of the generalized MAL systems.

Let us join each elementary process with its reverse process and represent the mechanism of diffusion by the pairs of mutually reverse processes:

$$
\sum_{i} \alpha_{r i}^{\mathrm{I}} A_{i}^{\mathrm{I}}+\sum_{i} \alpha_{r i}^{\mathrm{II}} A_{i}^{\mathrm{II}} \rightleftharpoons \sum_{i} \beta_{r i}^{\mathrm{I}} A_{i}^{\mathrm{I}}+\sum_{i} \beta_{r i}^{\mathrm{II}} A_{i}^{\mathrm{II}} .
$$

All the quantities for the direct process we mark by the upper index ${ }^{+}$and for the reverse process by the upper index ${ }^{-}$. The simple algebraic relations hold (see also (2.39):

$$
\alpha_{r i}^{\mathrm{I}, \mathrm{II} \mp}=\beta_{r i}^{\mathrm{I}, \mathrm{II} \pm} \text { and } \gamma_{r i}^{\mathrm{I}, \mathrm{II} \mp}=-\gamma_{r i}^{\mathrm{I}, \mathrm{II} \pm} .
$$

Therefore,

$$
\alpha_{r i}^{\mathrm{I}+}+\alpha_{r i}^{\mathrm{II}+}=\beta_{r i}^{\mathrm{I}-}+\beta_{r i}^{\mathrm{II}-}=\alpha_{r i}^{\mathrm{I}-}+\alpha_{r i}^{\mathrm{II}-}=\beta_{r i}^{\mathrm{I}+}+\beta_{r i}^{\mathrm{II}+} .
$$

Due to the principle of detailed balance,

$$
\phi_{r}^{+}=\phi_{r}^{-}=\phi_{r} .
$$

The reaction rates are

$$
\begin{aligned}
& w_{r}^{+}\left(c^{\mathrm{I}}, c^{\mathrm{II}}\right)=\phi_{r} \exp \left(\sum_{i} \alpha_{r i}^{\mathrm{I}} \frac{\mu_{i}^{\mathrm{I}}}{R T}+\sum_{i} \alpha_{r i}^{\mathrm{II}} \frac{\mu_{i}^{\mathrm{II}}}{R T}\right), \\
& w_{r}^{+}\left(c^{\mathrm{I}}, c^{\mathrm{II}}\right)=\phi_{r} \exp \left(\sum_{i} \beta_{r i}^{\mathrm{I}} \frac{\mu_{i}^{\mathrm{I}}}{R T}+\sum_{i} \beta_{r i}^{\mathrm{II}} \frac{\mu_{i}^{\mathrm{II}}}{R T}\right),
\end{aligned}
$$

The cell-jump model gives us (3.43)

$$
\begin{aligned}
J=\sum_{r} J_{r} & =-\sum_{r} \gamma_{r}\left(w_{r}^{+}\left(c^{\mathrm{I}}, c^{\mathrm{II}}\right)-w_{r}^{-}\left(c^{\mathrm{I}}, c^{\mathrm{II}}\right)\right) \\
=\sum_{r} \gamma_{r} \phi_{r} & \left(\exp \left(\sum_{i} \alpha_{r i}^{\mathrm{I}} \frac{\mu_{i}^{\mathrm{I}}}{R T}+\sum_{i} \alpha_{r i}^{\mathrm{II}} \frac{\mu_{i}^{\mathrm{II}}}{R T}\right)\right. \\
& \left.-\exp \left(\sum_{i} \beta_{r i}^{\mathrm{I}} \frac{\mu_{i}^{\mathrm{I}}}{R T}+\sum_{i} \beta_{r i}^{\mathrm{II}} \frac{\mu_{i}^{\mathrm{II}}}{R T}\right)\right) .
\end{aligned}
$$

In the first order approximation, we get analogously to (2.41):

$$
\begin{aligned}
& J_{r i}=-l \phi_{r} \exp \left(\sum_{j}\left(\alpha_{r j}^{\mathrm{I}}+\alpha_{r j}^{\mathrm{II}}\right) \frac{\mu_{j}^{\mathrm{II}}}{R T}\right) \sum_{j} \gamma_{r i} \gamma_{r j} \nabla\left(\frac{\mu_{j}^{\mathrm{II}}}{R T}\right), \\
& J_{r}=-l \phi_{r} \exp \left(\frac{\left(\alpha_{r}^{\mathrm{I}}+\alpha_{r}^{\mathrm{II}}, \mu\right)}{R T}\right) \gamma_{r}\left(\gamma_{r}, \nabla\left(\frac{\mu}{R T}\right)\right) .
\end{aligned}
$$


This expression for $J_{r}$ has the form of the multicomponent Teorell formula with the symmetric matrix of coefficients. This symmetry for nonlinear diffusion gives us the generalization of the Onsager reciprocal symmetry $[83,84]$. We represented the nonlinear multicomponent diffusion a sum of elementary processes. For each elementary process

$$
J_{r i}=-d_{r} \sum_{j} \gamma_{r i} \gamma_{r j} \nabla\left(\frac{\mu_{j}^{\mathrm{II}}}{R T}\right),
$$

where the scalar coefficient

$$
d_{r}=l \phi_{r} \exp \left(\sum_{j}\left(\alpha_{r j}^{\mathrm{I}}+\alpha_{r j}^{\mathrm{II}}\right) \frac{\mu_{j}^{\mathrm{II}}}{R T}\right)>0
$$

is, from the thermodynamic point of view, an almost arbitrary positive quantity (because it includes the kinetic factor $\phi_{r}$ ). "Almost" here means that some conditions of zero values (and the orders of these zeros) at the boundary when some of $c_{i}=0$ are prescribed by the factor

$$
\exp \left(\sum_{j}\left(\alpha_{r j}^{\mathrm{I}}+\alpha_{r j}^{\mathrm{II}}\right) \frac{\mu_{j}^{\mathrm{II}}}{R T}\right)
$$

and the logarithmic singularity of $\mu_{i}$ when $c_{i} \rightarrow 0$.

The internal symmetry of this formula makes the dissipation inequality obvious: in a bounded domain $V$ with smooth boundary and without fluxes through boundary for isothermal conditions

$$
\begin{aligned}
\frac{\mathrm{d} \mathbf{F}}{\mathrm{d} t} & =R T \int_{V} \sum_{j}\left(\nabla_{x}\left(\frac{\mu_{j}}{R T}\right), J_{j}\right) \mathrm{d} x \\
& =-l \sum_{r} \phi_{r} \exp \left(\frac{\left(\alpha_{r}^{\mathrm{I}}+\alpha_{r}^{\mathrm{II}}, \mu\right)}{R T}\right)\left(\gamma_{r}, \nabla\left(\frac{\mu}{R T}\right)\right)^{2} \leq 0 .
\end{aligned}
$$

\subsubsection{Generalization: Non-isothermal Processes}

Extension of the generalized MAL (3.30) on the non-isothermal processes is quite simple. Let us follow the paper [10] and include the "energetic component" $A_{U}$ in the list of components. Instead of the stoichiometric equations (2.1), (3.18) we get:

$$
\sum_{i} \alpha_{r i} A_{i}+\alpha_{Q} A_{U} \rightarrow \sum_{i} \beta_{r i} A_{i}+\beta_{Q} A_{U}
$$

The corresponding macroscopic extensive variable for $A_{U}$ is the internal energy $U$ with the density ("concentration") $u$. To consider energy as the additional extensive variable, we should take the main thermodynamic potential for the isolated system from the entropic series. This is the entropy $S$ :

$$
\mathrm{d} S=\frac{1}{T} \mathrm{~d} U+\frac{P}{T} \mathrm{~d} V-\sum_{i} \frac{\mu_{i}}{T} \mathrm{~d} N_{i} .
$$


Let us extend the formulas for the generalized kinetics by additional component and take $-\frac{1}{R T}$ instead of $\frac{\mu_{i}}{R T}$. All the formulas including the dissipation inequalities remain the same.

In isolated (isochoric) systems, $\dot{U}=0$ and

$$
\dot{N}_{i}=\sum_{r} \gamma_{r i} w_{r}
$$

where

$$
w_{r}=\phi_{r} \exp \left[\left(\alpha_{r}, \frac{\mu}{R T}\right)-\frac{\alpha_{Q}}{R T}\right] .
$$

For transport processes, conservation of energy gives the following relations:

$$
\alpha_{Q}^{\mathrm{I}}+\alpha_{Q}^{\mathrm{II}}=\beta_{Q}^{\mathrm{I}}+\beta_{Q}^{\mathrm{II}} .
$$

The space gradient of $-\frac{1}{R T}$ enters the multicomponent Teorell formula as an additional force and the gradients of $\frac{\mu_{i}}{R T}$ also enter the formulas for the heat flux.

In particular, the simplest mechanism of transport,

$$
\alpha_{Q} A_{U}^{\mathrm{I}} \rightleftharpoons \alpha_{Q} A_{U}^{\mathrm{II}},
$$

generates the Fourier law:

$$
J_{Q}=-l \alpha_{Q} \phi \exp \left(-\frac{\alpha_{Q}}{R T}\right) \nabla\left(-\frac{1}{R T(x)}\right)=-\lambda(T) \nabla T
$$

The thermodynamic consideration cannot produce the temperature dependence of the thermal conductivity $\lambda(T)>0$. From the thermodynamic point of view, $\phi$ here is an arbitrary positive quantity. The problem of temperature dependence of $\lambda$ and its relations with other constants like diffusivity is widely discussed from the kinetic point of view [77]. For computing thermal conductivity various methods were developed including direct simulation and the Green-Kubo approach [63]. These methods were compared in [93].

Thermodynamics may give the relations between different coefficients. For example, the principle of detailed balance produces the multicomponent Teorell formula with the symmetric matrix of coefficients (3.58). The nonlinear reciprocal relations (3.59) could be automatically extended to the heat flux: just use the heat flux $J_{Q}$ as additional flux and $-1 / R T$ instead $\mu_{i} / R T$ for the component $A_{U}$.

More relations we get for the specific mechanisms of transport. For example, for the activation mechanism of diffusion

$$
A^{\mathrm{I}}+\alpha_{Q} A_{U}^{\mathrm{I}} \rightleftharpoons A^{\mathrm{II}}+\alpha_{Q} A_{U}^{\mathrm{II}}
$$

the fluxes are:

$$
\begin{aligned}
& J_{A}=-l \phi \exp \left(\frac{\mu-\alpha_{Q}}{R T}\right)\left[\nabla\left(\frac{\mu}{R T}\right)+\alpha_{Q} \nabla\left(-\frac{1}{R T}\right)\right], \\
& J_{Q}=-l \phi \exp \left(\frac{\mu-\alpha_{Q}}{R T}\right)\left[\alpha_{Q} \nabla\left(\frac{\mu}{R T}\right)+\alpha_{Q}^{2} \nabla\left(-\frac{1}{R T}\right)\right],
\end{aligned}
$$


or in the matrix form

$$
\left(\begin{array}{c}
J_{A} \\
J_{Q}
\end{array}\right)=-l \phi \exp \left(\frac{\mu-\alpha_{Q}}{R T}\right)\left(\begin{array}{cc}
1 & \alpha_{Q} \\
\alpha_{Q} & \alpha_{Q}^{2}
\end{array}\right)\left(\begin{array}{c}
\nabla \\
\nabla\left(\frac{\mu}{R T}\right) \\
\left.-\frac{1}{R T}\right)
\end{array}\right) .
$$

For the mechanism (3.62), the heat flux $J_{Q}$ is proportional to the diffusion flux $J_{A}$ with the coefficient $\alpha_{Q}$, that is the activation heat. In this activation mechanism, the activation heat travels with the particle from the cell I to the cell II. We can, for example, assume different behavior of the activation heat: let $\beta_{Q}$ distribute symmetrically after the diffusion jump:

$$
A^{\mathrm{I}}+\alpha_{Q} A_{U}^{\mathrm{I}} \rightleftharpoons A^{\mathrm{II}}+\frac{1}{2} \alpha_{Q} A_{U}^{\mathrm{I}}+\frac{1}{2} \alpha_{Q} A_{U}^{\mathrm{II}} .
$$

For this mechanism, $\gamma_{U}=-\frac{1}{2} \alpha_{U}$ and

$$
\left(\begin{array}{c}
J_{A} \\
J_{Q}
\end{array}\right)=-l \phi \exp \left(\frac{\mu-\alpha_{Q}}{R T}\right)\left(\begin{array}{cc}
1 & \frac{1}{2} \alpha_{Q} \\
\frac{1}{2} \alpha_{Q} & \frac{1}{4} \alpha_{Q}^{2}
\end{array}\right)\left(\begin{array}{c}
\nabla\left(\frac{\mu}{R T}\right) \\
\nabla\left(-\frac{1}{R T}\right)
\end{array}\right) .
$$

The heat flux $J_{Q}$ should be supplemented by the heat transport together with particles, $\sum_{i} \mu_{i} J_{i}$, [70]. The total heat flux is

$$
\mathbf{q}=J_{U}=J_{Q}+\sum_{i} \mu_{i} J_{i} .
$$

To describe the energy balance properly we have to include the work of various forces. The proper framework for modeling of the energy transport gives continuum mechanics. In its simplest form, fluid mechanics, we present these equation in the next Section.

\subsection{Momentum and Center of Mass Conservation}

\subsubsection{Mass Transfer and Heat Transfer}

In this subsection, we briefly discuss coupling of the diffusion and thermal conductivity with fluid dynamics. The heat and mass transfer should satisfy the general laws of mechanics and, in particular, does not violate the Newton laws. The diffusion and heat transfer equations do not present the complete theory and should be included into the context of continuum mechanics.

First of all, let us introduce the mass average velocity. Let $m_{i}$ be the mass of mole (grammolecule) for the component $A_{i}$. For each diffusion flux $J_{i}$ the associated flux of mass is $m_{i} J_{i}$. We introduced the fluxes $J_{i}$ with respect to a frame, which is connected to our cells. For continuum motion, this frame should also move and we have to introduce the velocity of the frame, $w$. The flux of $A_{i}$ associated with $\mathbf{w}$ is $c_{i} \mathbf{w}$. The corresponding flux of mass is $m_{i} c_{i} \mathbf{w}$. The total flux of $A_{i}$ caused by diffusion and the frame motion is

$$
\check{J}_{i}=J_{i}+c_{i} \mathbf{w} .
$$

The mass density is

$$
\rho=\sum_{i} m_{i} c_{i}
$$


the momentum density is

$$
\sum_{i} m_{i} \check{J}_{i}
$$

the average mass velocity is

$$
\mathbf{v}=\frac{\sum_{i} m_{i} \breve{J}_{i}}{\sum_{i} m_{i} c_{i}}=\mathbf{w}+\frac{\sum_{i} m_{i} J_{i}}{\sum_{i} m_{i} c_{i}} .
$$

Both the frame velocity $\mathbf{w}$ and the average mass velocity $\mathbf{w}$ are unknown. They are connected by the simple identity

$$
\mathbf{v}=\mathbf{w}+\frac{\sum_{i} m_{i} J_{i}}{\sum_{i} m_{i} c_{i}}
$$

where the individual diffusion fluxes $J_{i}$ are given by the mechanism of diffusion.

The standard definition of the diffusion fluxes includes fluxes in the local center of mass frame where the average mass velocity is zero. Therefore, let us introduce the "proper fluxes":

$$
\mathcal{J}_{i}=\check{J}_{i}-\mathbf{v} c_{i}=\check{J}_{i}-c_{i} \frac{\sum_{i} m_{i} \check{J}_{i}}{\sum_{i} m_{i} c_{i}}=J_{i}-c_{i} \frac{\sum_{i} m_{i} J_{i}}{\sum_{i} m_{i} c_{i}} .
$$

These fluxes do not depend on the frame velocity. They are not independent and are connected by the momentum conservation: the total momentum is zero,

$$
\sum m_{i} \mathcal{J}_{i}=0
$$

The heat flux in the local center of mass system is

$$
\mathcal{J}_{U}=\left(J_{Q}-\frac{\sum_{i} m_{i} J_{i}}{\sum_{i} m_{i} c_{i}} u\right)+\sum_{i} \mu_{i} \mathcal{J}_{i}
$$

where $J_{Q}$ is given by the transport processes mechanism.

For the energy flux, the standard approach [70] gives

$$
\mathbf{v}\left(\frac{\rho v^{2}}{2}+u+P\right)+\mathcal{J}_{U}+\text { viscosity terms . }
$$

The conservation laws give:

$$
\begin{aligned}
& \partial_{t} \rho+\operatorname{div}(\rho \mathbf{v})=0 \\
& \partial_{t}(\rho \mathbf{v})+\operatorname{div}(\rho \mathbf{v} \otimes \mathbf{v})+\nabla P=\operatorname{div} \sigma \\
& \partial_{t}\left(\frac{\rho v^{2}}{2}+u\right)+\operatorname{div}\left[\mathbf{v}\left(\frac{\rho v^{2}}{2}+u+P\right)+\mathcal{J}_{U}\right]=\sigma: \nabla \mathbf{v} \\
& \partial_{t} c_{i}+\operatorname{div}\left(\mathbf{v} c_{i}+\mathcal{J}_{i}\right)=0
\end{aligned}
$$


Here, $\sigma$ is the viscous stress tensor and $\sigma: \nabla \mathbf{v}=\sum_{i j} \sigma_{i j}\left(\partial v_{i} / \partial x_{j}\right)$. The pressure $P$ should be defined in accordance with thermodynamic properties of the mixture, for example,

$$
P=-\frac{\partial F}{\partial V}=\sum_{i} c_{i} \frac{\partial f(c, T)}{\partial c_{i}}-f(c, T),
$$

where $f(c, T)$ is the density of the free energy.

The viscous stress tensor should be derived in (3.68) from the additional closure assumption. For the Newtonian liquid,

$$
\sigma_{i j}=\mu\left(\frac{\partial v_{i}}{\partial x_{j}}+\frac{\partial v_{j}}{\partial x_{i}}\right)+\delta_{i j}\left(\zeta-\frac{2}{3} \mu\right) \operatorname{div} \mathbf{v},
$$

where $\mu$ (here and only here) is the shear viscosity and $\zeta$ is the bulk viscosity.

The individual equations in (3.68) are not independent. For example, the sum of the equations for conservation of $N_{i}$ with coefficients $m_{i}$ gives us the first equation, the conservation of mass.

The elastic energy and the various viscoelastic terms may be added to this picture. This is necessary to do and it is in our future plans.

\subsubsection{Mechanisms of Transport and the General Forms of Macroscopic Equation}

We developed a formalism of the mechanism of diffusion and heat conduction represented by the system of stoichiometric equations with the simple kinetic law $\exp (\alpha, \mu / R T)$ and the balance condition (complex balance for the general Markov microscopic kinetics and detailed balance for systems with microreversibility). This formalism produces equations which are particular cases of the general nonequilibrium thermodynamic equations $[20,85]$. It is a very simple task to demonstrate that our transport equations are particular cases of the GENERIC formalism [56, 85]. Due to this two-generator formalism, evolution of any smooth function $A$ of the state variables $x$ is given by

$$
\frac{\mathrm{d} A}{\mathrm{~d} t}=\{A, E\}+[A, S]
$$

where $E$ and $S$ are the total energy and entropy, and $\{\cdot, \cdot\}$ and $[\cdot, \cdot]$ are Poisson and dissipative brackets, respectively.

The formulas for fluxes produced in this Section have the form of dissipative brackets:

$$
[A, S]=\frac{\delta A}{\delta x} M \frac{\delta S}{\delta x}
$$

where $M$ is a symmetric positively semidefinite operator, "the friction matrix".

The general form of the "dissipative brackets with constraints" in application to multicomponent diffusion was produced very recently [86]. The flux of the $i$ th component $J_{i}$ in that formalism was presented by formula (54) [86]:

$$
J_{i}=-\sum_{j} \Lambda_{i j}^{c}\left[\nabla\left(\frac{\mu_{j}}{T}\right)+\Lambda_{j}^{\prime} \nabla\left(-\frac{1}{T}\right)\right] .
$$


Our formulas belong to this type and give particular expressions for coefficients $\Lambda$.

In the paper [86] a precise comparison of this formula with the classical expressions from [20] was presented and the equivalence of these general forms was proven. Now, we can just refer to these results.

In addition to the general form, our approach gives the possibility to build the model from the elementary processes. This construction also satisfies the "constrains" (conservation laws) of diffusion because these conservation laws are implemented in the algebra of the stoichiometric coefficients (2.22).

\section{Conclusion}

Chemical kinetics gave rise to the very seminal approach of the modeling of processes. This is, the stoichiometric algebra supplemented by the simple kinetic law. The results of this invention are now applied in may areas of science, from particle physics to sociology. In our work we extend the area of applications to nonlinear multicomponent diffusion.

We demonstrated, how the mechanism based approach to multicomponent diffusion can be included within the general thermodynamic framework and proved the corresponding dissipation inequalities. To satisfy the thermodynamic restrictions, the kinetic law of an elementary process cannot have an arbitrary form. For the general kinetic law $\phi \exp (\alpha, \mu / R T)$ (the generalized Mass Action Law), additional conditions on the set of kinetic factors $\phi$ are needed. There are two main sets of these conditions. The historically first of them, the condition of detailed balance, follows from the special property of the underlying microscopic dynamics, microreversibility. It was used by Boltzmann for his equation and then by many researchers like Wegscheider [113], Einstein [25], and Onsager [83]. The second and more general property we now call "semi-detailed balance" or "complex balance" was proposed by Boltzmann in his discussion with Lorentz. The theoretic principles underneath these conditions were discovered by Stueckelberg in 1952 [97] in application to the Boltzmann equation. It received the name "complex balance" ten years later in the works of Horn and Jackson [62] and Feinberg [30]. Recently, it was demonstrated how this condition can be deduced from the quasiequilibrium and quasi steady state approximations from Markov kinetics [54].

Explicit formulas for nonlinear multicomponent diffusion combined from elementary processes can help in the practice of modeling (and already helped [45]). The cell-jump formalism gives an intuitively clear representation of the elementary transport processes and, at the same time, produces kinetic finite elements, a tool for numerical simulation.

There remain many questions for the future work:

- It is necessary to extend the experience of modeling of real systems;

- The analysis of diffusion in solids should be properly coupled with the mechanics of solids. The detailed quantitative theory of the Kirkendall effect may be a proper challenge here;

- The mechanism representation should be extended to viscosity and viscoelasticity; 
- The kinetic finite elements approach should be modified and extended to include continuum mechanics, perhaps, by proper joining with the lattice Boltzmann models approach;

- Possible stoichiometric mechanisms of anomalous diffusion should be studied.

\section{References}

[1] G.I. Barenblatt. On some unsteady motions of a liquid or a gas in a porous medium. Prikl. Mat. Mekh., 16 (1952), 67-78.

[2] G.I. Barenblatt, Y.B. Zeldovich. Self-similar solutions as intermediate asymptotics. Ann. Rev. Fluid Mech., 4 (1972), 285-312.

[3] L. Bertini, C. Landim, S. Olla. Derivation of Cahn-Hilliard Equations from GinzburgLandau Models. J. Stat. Phys., 88 (1997), Nos. 1/2, 365-381.

[4] T. Blesgen, U. Weikard. Multi-component Allen-Cahn equation for elastically stressed solids. Electron. J. Diff. Eqns., 89 (2005), 1-17.

[5] M. Boudart. From the century of the rate equation to the century of the rate constants: a revolution in catalytic kinetics and assisted catalyst design. Catal. Lett., 65 (2000), 1-3.

[6] L. Boltzmann. Lectures on gas theory. U. of California Press, Berkeley, CA, 1964.

[7] G.E. Briggs, J.B.S. Haldane. A note on the kinetics of enzyme action. Biochem. J., 19 (1925), 338-339.

[8] R.A. Brownlee, A.N. Gorban, J. Levesley. Nonequilibrium entropy limiters in lattice Boltzmann methods. Physica A, 387 (2008), 385-406.

[9] V.I. Bykov, S.E. Gilev, A.N. Gorban, G.S. Yablonskii. Imitation modeling of the diffusion on the surface of a catalyst. Dokl. Akad. Nauk SSSR, 283 (1985), 1217-1220.

[10] V.I. Bykov, A.N. Gorban, G.S. Yablonskii. Description of non-isothermal reactions in terms of Marcelin-De-Donder Kinetics and its generalizations. React. Kinet. Catal. Lett. 20 (1982), 261-265.

[11] J.W. Cahn. Free energy of a nonuniform system. II. Thermodynamic basis. J. Chem. Phys., 30 (1959), 1121-1124.

[12] J.W. Cahn, J.E. Hilliard. Free energy of a nonuniform system. I. Interfacial energy. J. Chem. Phys., 28 (1958), 258-266.

[13] J.W. Cahn, J.E. Hilliard. Spinodal decomposition: A reprise. Acta Metallurgica, 19 (1971), $151-161$. 
[14] H.B. Callen. Thermodynamics and an introduction to themostatistics (2nd ed.). John Wiley \& Sons, NY, 1985.

[15] C. Cercignani, M. Lampis. On the H-theorem for polyatomic gases. J. Stat. Phys., 26 (1981), 795-801.

[16] B. Chopard, M. Droz. Cellular automata modeling of physical systems. Cambridge University Press, Cambridge, UK, 1998.

[17] R. Clausius. Über verschiedene für die Anwendungen bequeme Formen der Hauptgleichungen der Wärmetheorie. Poggendorffs Annalen der Physic und Chemie, 125 (1865), 353400 .

[18] A.J. Chorin, O.H. Hald, R. Kupferman. Optimal prediction with memory. Physica D, 166 (2002), 239-257.

[19] F. Coester. Principle of detailed balance. Phys. Rev., 84, 1259 (1951)

[20] S.R. De Groot, P. Mazur. Non-equilibrium Thermodynamics. North-Holland, Amsterdam, 1962.

[21] K. Denbigh. The principles of chemical equilibrium. Cambridge University Press, Cambridge, UK, 1981.

[22] S. Dushman, I. Langmuir. The diffusion coefficient in solids and its temperature coefficient. Phys. Rev., 20 (1922), 113.

[23] P. Ehrenfest, T. Ehrenfest-Afanasyeva. Begriffliche Grundlagen der statistischen Auffassung in der Mechanik. In: Mechanics Enziklopädie der Mathematischen Wissenschaften, Vol. 4. Leipzig, 1911. (Reprinted in: Ehrenfest, P., Collected Scientific Papers. North-Holland, Amsterdam, 1959, pp. 213-300.)

[24] A. Einstein. Über die von der molekularkinetischen Theorie der Wärme geforderte Bewegung von in ruhenden Flüssigkeiten suspendierten Teilchen. Ann. Phys., 17 (1905) 549-560.

[25] A. Einstein. Strahlungs-Emission und-Absorption nach der Quantentheorie. Verhandlungen der Deutschen Physikalischen Gesellschaft, 18 (1916), No. 13/14, Braunschweig, Vieweg, 318-323.

[26] C.M. Elliott, Z. Songmu. On the Cahn-Hilliard equation. Arch. Rat. Mechan. Anal., 96 (1986) 339-357.

[27] C.M. Elliott, A.M. Stuart. The global dynamics of discrete semilinear parabolic equations. SIAM J. Numer. Anal., 30 (1993), 1622-1663.

[28] H. Eyring. The activated complex in chemical reactions. J. Chem. Phys., 3 (1935), 107-115. 
[29] M. Feinberg. On chemical kinetics of a certain class. Arch. Rat. Mechan. Anal., 46 (1972), $1-41$.

[30] M. Feinberg. Complex balancing in general kinetic systems. Arch. Rat. Mechan. Anal., 49 (1972), 187-194.

[31] R.F. Feynman. Simulating physics with computers. Internat. J. Theor. Phys., 21 (1982), 467488.

[32] A. Fick. Über Diffusion. Poggendorff's Annalen der Physik und Chemie, 94 (1855), 59-86.

[33] R.A. Fisher. The genetical theory of natural selection. Oxford University Press, Oxford, 1930.

[34] F.C. Frank, D. Turnbull. Mechanism of diffusion of copper in Germanium. Phys. Rev., 104 (1956), 617-618.

[35] A. Fratzl, O. Penrose, J.L. Lebowitz. Modelling of phase separation in alloys with coherent elastic misfit. J. Stat. Phys., 95 (1999), 1429-1503.

[36] J. Frenkel. Theorie der Adsorption und verwandter Erscheinungen. Zeitschrift für Physik, 26 (1924), 117-138

[37] J. Frenkel. Über die Wärmebewegung in festen und flüssigen Körpern. Zeitschrift für Physik, 35 (1925), 652-669.

[38] G.F. Gause. The struggle for existence. Williams \& Wilkins, Baltimore, 1934.

[39] J.W. Gibbs. On the equilibrium of heterogeneous substance. Trans. Connect. Acad., 18751876, 108-248; 1877-1878, 343-524.

[40] D.T. Gillespie. Exact stochastic simulation of coupled chemical reactions. J. Phys. Chem., 81 (1977), 2340-2361.

[41] D.T. Gillespie. Stochastic simulation of chemical kinetics. Annu. Rev. Phys. Chem., 58 (2007), 35-55.

[42] A.N. Gorban. Equilibrium encircling. Equations of chemical kinetics and their thermodynamic analysis. Nauka, Novosibirsk, 1984.

[43] A.N. Gorban. Singularities of transition processes in dynamical systems: qualitative theory of critical delays. Electron. J. Diff. Eqns., Monograph 05, 2004. E-print: http://arxiv.org/abs/chao-dyn/9703010, 1997.

[44] A.N. Gorban. Basic types of coarse-graining. In: Model reduction and coarse-graining approaches for multiscale phenomena, Ed. by A.N. Gorban, N. Kazantzis, I.G. Kevrekidis, H.C. Öttinger, C. Theodoropoulos. Springer, Berlin-Heidelberg-New York, 2006, 117-176. E-print: http://arxiv.org/abs/cond-mat/0602024, 2006. 
[45] A.N. Gorban, V.I. Bykov, G.S. Yablonskii. Macroscopic clusters induced by diffusion in catalytic Oxidation Reactions. Chem. Eng. Sci., 35 (1980), 2351-2352.

[46] A.N. Gorban, V.I. Bykov, G.S. Yablonskii. Essays on chemical relaxation. Novosibirsk, Nauka Publ., 1986.

[47] A.N. Gorban, P.A. Gorban, G. Judge. Entropy: The Markov ordering approach. Entropy, 12 (2010), 1145-1193. E-print: http://arxiv.org/abs/1003.1377, 2010.

[48] A.N. Gorban, I.V. Karlin, H.C. Öttinger, L.L. Tatarinova. Ehrenfest's argument extended to a formalism of nonequilibrium thermodynamics. Phys.Rev. E, 63 (2001), 066124.

[49] A.N. Gorban, I.V. Karlin. Uniqueness of thermodynamic projector and kinetic basis of molecular individualism. Physica A, 336 (2004), 391-432. E-print: http://arxiv.org/abs/cond-mat/0309638, 2003.

[50] A.N. Gorban, I.V. Karlin. Method of invariant manifold for chemical kinetics. Chem. Eng. Sci., 58 (2003), 4751-4768.

[51] A.N. Gorban, I.V. Karlin. Invariant manifolds for physical and chemical kinetics. Lect. Notes Phys. 660, Springer, Berlin, Heidelberg, 2005.

[52] A.N. Gorban, I.V. Karlin., P. Ilg, H.C. Öttinger. Corrections and enhancements of quasiequilibrium states. J. Non-Newtonian Fluid Mech., 96 (2001), 203-219.

[53] A.N. Gorban, H.P. Sargsyan. Mass action law for nonlinear multicomponent diffusion and relations between its coefficients. Kinetics and Catalysis, 27 (1986), 527.

[54] A.N. Gorban, M. Shahzad. QE+QSS for derivation of kinetic equations and stiffness removing. E-print: http://arxiv.org/abs/1008.3296, 2010.

[55] T. Graham. The Bakerian lecture: on the diffusion of liquids. Phil. Trans. R. Soc. Lond., 140 (1) (1850), 1-46; doi:10.1098/rstl.1850.0001.

[56] M. Grmela, H.C. Öttinger. Dynamics and thermodynamics of complex fluids. I. Development of a general formalism. Phys. Rev. E, 56 (1997), 6620-6632.

[57] W.S.C. Gurney, R.M. Nisbet. A note on nonlinear population transport. J. Theor. Biol., 56 (1976), 249-251.

[58] M.E. Gurtin. Generalized Ginzburg-Landau and Cahn-Hilliard equations based on a microforce balance. Physica D, 92 (1996), 178-192.

[59] I. Gyarmati. Non-equilibrium thermodynamics. Field theory and variational principles. Springer, Berlin, 1970.

[60] W. Heitler. Quantum Theory of Radiation. Oxford University Press, London, 1944. 
[61] R. Hengeveld. Dynamics of biological invasions. Chapman and Hall, London, 1989.

[62] F. Horn, R. Jackson. General mass action kinetics. Arch. Rat. Mechan. Anal., 47 (1972), 81-116.

[63] W.G. Hoover. Computational statistical mechanics. Elsevier, Amsterdam, 1991.

[64] I.V. Karlin, A.N. Gorban, S. Succi, V. Boffi. Maximum entropy principle for lattice kinetic equations. Phys. Rev. Lett., 81 (1998), 6-9.

[65] L.B. Kier, P.G. Seybold, Ch-K. Cheng. Modeling chemical systems using cellular automata. Dordrecht, The Netherlands, 2005.

[66] J.F. Kincaid, H. Eyring, A.E. Stearn. The theory of absolute reaction rates and its application to viscosity and diffusion in the liquid State. Chem. Rev., 28, (1941), 301-365.

[67] E.O. Kirkendall. Diffusion of zinc in alpha brass. Trans. Am. Inst. Min. Metall. Eng., 147 (1942), 104-110.

[68] A.B. Kudryavtsev, R.F. Jameson, W. Linert. The law of mass action. Springer, Berlin Heidelberg - New York, 2001.

[69] K.J. Laidler, A. Tweedale. The current status of Eyring's rate theory. In: Advances in Chemical Physics: Chemical dynamics: Papers in honor of Henry Eyring, Volume 21 (eds J. O. Hirschfelder and D. Henderson). John Wiley \& Sons, Inc., Hoboken, NJ, USA, 2007.

[70] L.D. Landau, E.M. Lifshitz. Fluid mechanics: Volume 6 (Course of theoretical physics). Butterworth-Heinemann, Oxford-Woburn, 1987.

[71] J.S. Langer, M. Bar-on, H.D. Miller. New computational method in the theory of spinodal decomposition. Phys. Rev. A, 11 (1975), 1417-1429.

[72] G. Lebon, D. Jou, J. Casas-Vázquez. Understanding non-equilibrium thermodynamics: Foundations, applications, Frontiers. Springer, Berlin, 2008.

[73] A.J. Lotka. Elements of physical biology. Williams and Wilkins, Baltimore, 1925.

[74] R.J.P. Lyon. Time aspects of geothermometry. Mining Eng., 11 (1959), 1145-1151.

[75] B.H. Mahan. Microscopic reversibility and detailed balance. An analysis. J. Chem. Educ., 52 (1975), 299-302.

[76] S. Maier-Paape, B. Stoth, T. Wanner. Spinodal decomposition for multicomponent cahnhilliard systems. J. Stat. Phys., 98 (2000), 871-896.

[77] E. McLaughlin. The Thermal conductivity of liquids and dense gases. Chem. Rev., 64 (1964), 389-428. 
[78] H. Mehrer. Diffusion in solids - fundamentals, methods, materials, diffusion-controlled processes. Textbook, Springer Series in Solid-State Sciences, Vol. 155, Springer, Berlin - Heidelberg - New York, 2007.

[79] H. Mehrer, N.A. Stolwijk. Heroes and highlights in the history of diffusion. Diffusion Fundamentals, 11 (2009), 1-32.

[80] L. Michaelis, M. Menten. Die Kinetik der Intervintwirkung. Biochemistry Zeitung, 49 (1913), 333-369.

[81] H. Nakajima. The discovery and acceptance of Kirkendall effect: The result of a short research career. JOM, 49 (1997), 15-19.

[82] T.N. Narasimhan. Energetics of the Kirkendall effect. Current Science, 93 (2007), 12571264.

[83] L. Onsager. Reciprocal relations in irreversible processes. I. Phys. Rev., 37 (1931), 405426.

[84] L. Onsager. Reciprocal relations in irreversible processes. II. Phys. Rev., 38 (1931), 22652279.

[85] H.C. Öttinger. Beyond equilibrium thermodynamics. Wiley-Blackwell, Hoboken, NJ, 2005.

[86] H.C. Öttinger. Constraints in nonequilibrium thermodynamics: General framework and application to multicomponent diffusion. J. Chem. Phys., 130 (2009), 114904.

[87] H.C. Öttinger, M. Grmela. Dynamics and thermodynamics of complex fluids. II. Illustrations of a general formalism. Phys. Rev. E, 56 (1997), 6633-6655.

[88] K. Oura, V.G. Lifshits, A.A. Saranin, A.V. Zotov, M. Katayama. Surface science: An introduction. Springer, Berlin - Heidelberg, 2003.

[89] S.V. Petrovskii, B.-L. Li. Exactly solvable models of biological invasion. Chapman \& Hall / CRC Press, Boca-Raton-London-New York-Washington D.C., 2006.

[90] J. Philibert. One and a half century of diffusion: Fick, Einstein, before and beyond. Diffusion Fundamentals, 2 (2005), 1.1-1.10.

[91] W.C. Roberts-Austen. Bakerian lecture on the diffusion in metals. Phil. Trans. Roy. Soc. A 187, (1896). Part I: Diffusion of Molten Metals. 383-403; Part II: Diffusion of Solid Metals. 404-415.

[92] D. Rothman, S. Zaleski. Lattice-gas models of phase separation: interfaces, phase transitions and multiphase flow. Rev. Mod. Phys., 66 (1994), 1417-1480.

[93] P.K. Schelling, S.R. Phillpot, P. Keblinski. Comparison of atomic-level simulation methods for computing thermal conductivity. Phys. Rev. B, 65 (2002), 144306. 
[94] N.N. Semenov. Some problems relating to chain reactions and to the theory of combustion. Nobel Lecture, December 11, 1956. In: Nobel lectures in chemistry 1942-1962. World Scientific, Hackensack, NJ, 1999.

[95] E. Seneta. Nonnegative matrices and Markov chains. Springer, New York, 1981.

[96] N. Shigesada, K. Kawasaki. Biological invasions: theory and practice. Oxford University Press, Oxford, 1997.

[97] E.C.G. Stueckelberg. Théorème H et unitarité de S. Helv. Phys. Acta, 25 (1952), 577-580.

[98] S. Succi. The lattice Boltzmann equation for fluid dynamics and beyond. Clarendon Press, Oxford, UK, 2001.

[99] S. Succi, I. Karlin, H. Chen. Role of the $H$ theorem in lattice Boltzmann hydrodynamic simulations (Colloquium). Rev. Mod. Phys., 74 (2002), 1203-1220.

[100] S. Succi, "Lattice Boltzmann at all-scales: from turbulence to DNA translocation", Mathematical Modelling Centre Distinguished Lecture, University of Leicester, Leicester, UK, $15^{\text {th }}$ November 2006.

[101] T. Teorell. Studies on the "diffusion effect" upon ionic distribution-I Some theoretical considerations. Proc. N. A. S. USA, 21 (1935), 152-161.

[102] T. Teorell. Studies on the diffusion effect upon ionic distribution-II Experiments on ionic accumulation. The Journal of General Physiology, 21 (1937), 107-122.

[103] T. Toffoli, N. Margolus. Cellular automata machines: A new environment for modeling. MIT Press, Cambridge, MA, 1987.

[104] C. Tuijn. On the history of models for solid-state diffusion. Defect and Diffusion Forum, 143-147 (1997), 11-20.

[105] N.G. Van Kampen. Nonlinear irreversible processes. Physica, 67 (1973), 1-22.

[106] P. Van Mieghem. Performance analysis of communications networks and systems. Cambridge University Press, Cambridge, 2006.

[107] J.H. Van’t Hoff. Etudes de dynamique chimique. Frederic Muller, Amsterdam, 1884.

[108] J.L. Vázquez. The porous medium equation. Mathematical Theory. Oxford University Press, Oxford, 2007.

[109] A.I. Volpert, S.I. Khudyaev. Analysis in classes of discontinuous functions and equations of mathematical physics. Nijoff, Dordrecht, 1985.

[110] V. Volterra. Variazioni e fluttuazioni del numero d'individui in specie animali conviventi. Mem. R. Accad. Naz. dei Lincei, 2 (1926), 31-113. 
[111] J. Von Neumann, A.W. Burks. Theory of self-reproducing automata. University of Illinois Press, Urbana, 1966.

[112] S. Watanabe. Symmetry of physical laws. Part I. Symmetry in space-time and balance theorems. Rev. Mod. Phys., 27 (1955), 26-39.

[113] R. Wegscheider. Über simultane Gleichgewichte und die Beziehungen zwischen Thermodynamik und Reactionskinetik homogener Systeme. Monatshefte für Chemie / Chemical Monthly, 32 (1911), 849-906.

[114] D.A. Wolf-Gladrow. Lattice-gas cellular automata and lattice Boltzmann models. Springer, 2000.

[115] S. Wolfram. A new kind of science. Wolfram Media, Champaign, IL, 2002.

[116] W.F.K. Wynne-Jones, H. Eyring. The absolute rate of reactions in condensed phases. J. Chem. Phys., 3 (1935), 492-502.

[117] G.S. Yablonskii, V.I. Bykov, A.N. Gorban, V.I. Elokhin. Kinetic models of catalytic reactions. Series "Comprehensive Chemical Kinetics", Vol. 32, Compton R.G. (ed.), Elsevier, Amsterdam, 1991.

[118] Y.B. Zeldovich. Proof of the uniqueness of the solution of the equations of the law of mass action. In: Selected Works of Yakov Borisovich Zeldovich; Volume 1, Ostriker, J.P., Ed. Princeton University Press, Princeton, NJ, USA, 1996; pp. 144-148. 